\title{
Ordinary state-based peridynamics for geometrically nonlinear analysis of plates
}

\section{Cong Tien Nguyen and Selda Oterkus*}

Department of Naval Architecture, Ocean and Marine Engineering, University of Strathclyde, Glasgow, G11XQ, UK

*Corresponding author: selda.oterkus@strath.ac.uk

\begin{abstract}
This study presents a novel ordinary state-based peridynamic model for geometrically nonlinear analysis of plates. The nonlinear strain energy density and nonlinear equations of motion for a plate in peridynamics are obtained based on the principle of virtual displacements by using Total Lagrange formulation. The numerical procedure for geometrically nonlinear analysis of a plate is also provided. The accuracy of the proposed nonlinear peridynamic model is validated by considering large deformations of a plate subjected to bending and a plate subjected to transverse shear forces. To further demonstrate the capabilities of the proposed nonlinear model, damages on a plate with a single crack subjected to stretching and tearing, a plate with two parallel cracks subjected to tearing, and a plate subjected to torsional loading are predicted.
\end{abstract}

Keywords: peridynamics, large deformations, plate, nonlinear, damage 


\section{Nomenclature}

\section{Latin Letters}

$$
\begin{aligned}
& a_{i p}, b_{i p}, d_{i p} \\
& a_{b}, b_{b}, d_{b} \\
& { }^{0} A_{(k)} \\
& C_{s h} \\
& { }^{t} \overline{\mathbf{b}}_{(k)} \\
& { }^{t} \bar{b}_{x(k)},{ }^{t} \bar{b}_{y(k)},{ }^{t} \bar{b}_{z(k)} \\
& { }_{0} \underline{\hat{e}}_{x x},{ }_{0} \underline{\hat{e}}_{y y},{ }_{0} \underline{\hat{e}}_{z z},{ }_{0} \underline{\hat{e}}_{x y},{ }_{0} \underline{\hat{e}}_{y z}, \\
& { }_{0} \underline{\hat{e}}_{x z} \\
& { }^{E} \\
& { }^{t} F_{x(k)},{ }^{t} F_{y(k)},{ }^{t} F_{z(k)} \\
& { }_{0}^{t} \bar{g}_{(k)(j)}
\end{aligned}
$$$$
g_{c}
$$$$
G_{c}
$$$$
h
$$$$
k_{s}
$$$$
\text { m }
$$$$
{ }^{t} \bar{m}_{x(k)},{ }^{t} \bar{m}_{y(k)}
$$$$
{ }^{t} M_{x(k)},{ }^{t} M_{y(k)}
$$$$
N_{c}
$$$$
{ }_{0}^{t+\Delta t} \hat{S}_{x x},{ }_{0}^{t+\Delta t} \hat{S}_{y y},{ }_{0}^{t+\Delta t} \hat{S}_{z z},{ }_{0}^{t+\Delta t} \hat{S}_{x y},
$$$$
{ }_{0}^{t+\Delta t} \hat{S}_{y z},{ }_{0}^{t+\Delta t} \hat{S}_{x z}
$$$$
{ }_{0}^{t} \hat{S}_{x x},{ }_{0}^{t} \hat{S}_{y y},{ }_{0}^{t} \hat{S}_{z z},{ }_{0}^{t} \hat{S}_{x y},{ }_{0}^{t} \hat{S}_{y z},{ }_{0}^{t} \hat{S}_{x z}
$$

PD constants for in-plane deformations.

PD constants for bending deformations.

Area of material point $k$ in the undeformed configuration.

PD constant for shear deformations.

Vector of external forces and moments per unit area at time $t$.

External forces per unit area at time $t$ of material point $k$.

Linear components of the incremental GL strain. This GL strain is measured with respect to the initial configuration.

Elastic modulus of materials.

External forces that were applied on material point $k$ at time $t$.

The energy release rate for the interaction between material points $k$ and $j$ at time $t$.

The average critical energy release rate for one interaction.

The critical energy release rate of the material.

The thickness of the plate.

Shear correction factor.

The time-independent mass matrix.

External moments per unit area at time $t$ of material point $k$.

External bending moments applied on material point $k$ at time $t$.

Total number of interactions passing through a unit crack area of $A_{0}=h \Delta x$.

Components of Second Piola-Kirchhoff (SPK) stress at time $t+\Delta t$. This stress is measured with respect to the initial configuration.

Components of Second Piola-Kirchhoff (SPK) stress at time $t$. This stress is measured with respect to the initial configuration. 


$$
\begin{aligned}
& { }_{0} \underline{\hat{S}}_{x x},{ }_{0} \underline{\hat{S}}_{y y},{ }_{0} \underline{\hat{S}}_{z z},{ }_{0} \underline{\hat{S}}_{x y},{ }_{0} \underline{\hat{S}}_{x z}, \\
& { }_{0} \underline{\hat{S}}_{y z} \\
& { }_{0}^{t} S_{i p(k)(j)} \\
& 0 \underline{\boldsymbol{S}}_{i p(k)(j)} \\
& { }_{0}^{t} \overline{\mathbf{t}}_{(k)(j)},{ }_{0}^{t} \overline{\mathbf{t}}_{(j)(k)} \\
& { }_{0}^{t} \bar{t}_{(k)(j)}^{u},{ }_{0}^{t} \bar{t}_{(k)(j)}^{v},{ }_{0}^{t} \bar{t}_{(k)(j)}^{w},{ }_{0}^{t} \bar{t}_{(k)(j)}^{\theta_{x}}, \\
& { }_{0}^{t} \bar{t}_{(k)(j)}^{\theta_{y}} \\
& { }^{t} \hat{\mathbf{u}}=\left[{ }^{t} \hat{u},{ }^{t} \hat{v},{ }^{t} \hat{w}\right]^{T} \\
& { }^{t+\Delta t} \hat{\mathbf{u}}=\left[{ }^{t+\Delta t} \hat{u},{ }^{t+\Delta t} \hat{v},{ }^{t+\Delta t} \hat{w}\right]^{T} \\
& { }^{t} \mathbf{u}=\left[{ }^{t} u,{ }^{t} v,{ }^{t} w\right]^{T} \\
& \underline{\hat{\mathbf{u}}}=\left[\begin{array}{lll}
\underline{\hat{u}} & \underline{\hat{v}} & \underline{\hat{w}}
\end{array}\right]^{T} \\
& \underline{\mathbf{u}}^{T} \\
& \underline{\mathbf{u}}=\left[\begin{array}{lll}
\underline{u} & \underline{v} & \underline{w}
\end{array}\right]^{T}
\end{aligned}
$$$$
{ }_{0}^{t} \hat{u}_{, x},{ }_{0}^{t} \hat{u}_{, y},{ }_{0}^{t} \hat{u}_{, z},{ }_{0}^{t} \hat{v}_{, x},{ }_{0}^{t} \hat{v}_{, y},{ }_{0}^{t} \hat{v}_{, z},
$$$$
{ }_{0}^{t} \hat{w}_{, x},{ }_{0}^{t} \hat{w}_{, y},{ }_{0}^{t} \hat{w}_{, z}
$$$$
{ }_{0}^{t} u_{, x},{ }_{0}^{t} u_{, y},{ }_{0}^{t} u_{, z},{ }_{0}^{t} v_{, x},{ }_{0}^{t} v_{, y},{ }_{0}^{t} v_{, z},
$$$$
{ }_{0}^{t} w_{, x},{ }_{0}^{t} w_{, y},{ }_{0}^{t} w_{, z}
$$$$
{ }_{0} \underline{\hat{u}}_{, x},{ }_{0} \underline{\hat{u}}_{, y},{ }_{0} \underline{\hat{u}}_{, z},{ }_{0} \underline{\hat{v}}_{, x},{ }_{0} \underline{\hat{v}}_{, y},
$$$$
{ }_{0} \underline{\hat{v}}_{, z},{ }_{0} \underline{\hat{w}}_{, x},{ }_{0} \underline{\hat{w}}_{, y},{ }_{0} \underline{\hat{w}}_{, z}
$$$$
{ }_{0} \underline{u}_{, x},{ }_{0} \underline{u}_{, y},{ }_{0} \underline{u}_{, z},{ }_{0} \underline{v}_{, x}, 0 \underline{v}_{, y},
$$$$
{ }_{0} \underline{v}_{, z},{ }_{0} \underline{w}_{, x},{ }_{0} \underline{w}_{, y},{ }_{0} \underline{w}_{, z}
$$$$
{ }^{t} \ddot{\hat{\mathbf{u}}} \text { and }{ }^{t+\Delta t} \ddot{\hat{\mathbf{u}}}
$$

Incremental SPK stress from time $t$ to time $t+\Delta t$. This incremental stress is measured with respected to the initial configuration.

Nonlinear bond stretch at time $t$ for in-plane deformations.

Incremental bond stretch for in-plane deformations.

Vectors of force densities at time $t$. These force densities are measured with respect to the initial configuration.

Force densities corresponding to five degrees of freedom, $u$ , $v, w, \theta_{x}, \theta_{y}$, of the plate. These force densities are measured with respect to the initial configuration.

Displacements at time $t$ of any point located at $\left({ }^{0} x,{ }^{0} y,{ }^{0} z\right)$ through the thickness.

Displacements at time $t+\Delta t$ of any point located at $\left({ }^{0} x,{ }^{0} y,{ }^{0} z\right)$ through the thickness.

Displacements at time $t$ of the material point located at $\left({ }^{0} x,{ }^{0} y, 0\right)$ on the mid-plane of the plate.

The incremental displacements from time $t$ to time $t+\Delta t$ of any point located at $\left({ }^{0} x,{ }^{0} y,{ }^{0} z\right)$ through the thickness.

Transpose of vector $\underline{\hat{\mathbf{u}}}$.

The incremental displacements from time $t$ to time $t+\Delta t$ of a material point located at $\left({ }^{0} x,{ }^{0} y, 0\right)$ on the mid-plane of the plate.

Derivatives of the displacements at time $t,\left({ }^{t} \hat{u},{ }^{t} \hat{v},{ }^{t} \hat{w}\right)$, of any point through the thickness with respect to the coordinate ${ }^{0} x,{ }^{0} y,{ }^{0} z$ in the initial configuration.

Derivatives of the displacements $\left({ }^{t} u,{ }^{t} v,{ }^{t} w\right)$ at time $t$ of a point located at the mid-plane with respect to the coordinate ${ }^{0} x,{ }^{0} y,{ }^{0} z$ in the initial configuration.

Derivatives of the incremental displacements $(\underline{\hat{u}}, \underline{\hat{v}}, \underline{\hat{w}})$ of any point through the thickness with respect to the coordinate ${ }^{0} x,{ }^{0} y,{ }^{0} z$ in the initial configuration.

Derivatives of the incremental displacements $(\underline{u}, \underline{v}, \underline{w})$ of a point located at the mid-plane with respect to the coordinate ${ }^{0} x,{ }^{0} y,{ }^{0} z$ in the initial configuration.

Vector of acceleration at time $t$ and $t+\Delta t$ of a material point located at $\left({ }^{0} x,{ }^{0} y,{ }^{0} z\right)$. 


$$
\begin{aligned}
& { }^{t} \ddot{\mathbf{u}} \text { and }{ }^{t+\Delta t} \ddot{\mathbf{u}} \\
& { }^{0} V \\
& W^{N L} \\
& \bar{W}^{N L} \\
& \bar{W}_{i p}^{N L} \\
& \bar{W}_{s h}^{N L} \\
& \bar{W}_{b}^{N L} \\
& \bar{W}_{(k)}^{N L P D} \\
& \bar{W}_{i p(k)}^{N L P D} \\
& \bar{W}_{s h(k)}^{N L P D} \\
& \bar{W}_{b(k)}^{N L P D} \\
& \left({ }^{0} x,{ }^{0} y,{ }^{0} z\right) \\
& \left({ }^{t} x,{ }^{t} y,{ }^{t} z\right) \\
& { }^{t+\Delta t} \mathfrak{R} \\
& \left.{ }^{N L}\right)
\end{aligned}
$$

\section{Greek Letters}

$\delta$

${ }_{0}^{t+\Delta t} \hat{\varepsilon}_{x x},{ }_{0}^{t+\Delta t} \hat{\varepsilon}_{y y},{ }_{0}^{t+\Delta t} \hat{\varepsilon}_{z z},{ }_{0}^{t+\Delta t} \hat{\varepsilon}_{x y}$, ${ }_{0}^{t+\Delta t} \hat{\varepsilon}_{x z},{ }_{0}^{t+\Delta t} \hat{\varepsilon}_{y z}$

${ }_{0}^{t} \hat{\varepsilon}_{x x},{ }_{0}^{t} \hat{\varepsilon}_{y y},{ }_{0}^{t} \hat{\varepsilon}_{z z},{ }_{0}^{t} \hat{\varepsilon}_{x y},{ }_{0}^{t} \hat{\varepsilon}_{x z},{ }_{0}^{t} \hat{\varepsilon}_{y z}$

${ }_{0} \underline{\hat{\varepsilon}}_{x x},{ }_{0} \underline{\hat{\varepsilon}}_{y y},{ }_{0} \hat{\hat{\varepsilon}}_{z z},{ }_{0} \underline{\hat{\varepsilon}}_{x y},{ }_{0} \hat{\boldsymbol{\varepsilon}}_{x z}$,

${ }_{0} \hat{\varepsilon}_{y z}$

${ }_{0} \underline{\hat{\eta}}_{x x},{ }_{0} \underline{\hat{\eta}}_{y y},{ }_{0} \underline{\hat{\eta}}_{z z},{ }_{0} \underline{\hat{\eta}}_{x y},{ }_{0} \underline{\hat{\eta}}_{x z}$

${ }_{0} \underline{\hat{\eta}}_{y z}$

$\underline{\theta}_{x}, \underline{\theta}_{y}$
Vector of accelerations at time $t$ and $t+\Delta t$ of a material point located at $\left({ }^{0} x,{ }^{0} y, 0\right)$ on the mid-plane.

Volume at the undeformed configuration.

Nonlinear strain energy density in classical continuum mechanics.

Nonlinear strain energy per unit area in classical continuum mechanics.

Nonlinear strain energy per unit area for in-plane deformations in classical continuum mechanics.

Nonlinear strain energy per unit area for shear deformations in classical continuum mechanics.

Nonlinear strain energy per unit area for bending deformations in classical continuum mechanics.

Nonlinear strain energy per unit area in PD.

Nonlinear strain energy per unit area in PD for in-plane deformations.

Nonlinear strain energy per unit area in PD for shear deformations.

Nonlinear strain energy per unit area in PD for bending deformations.

Local coordinates of the plate in the undeformed configuration.

Local coordinates of the plate in the deformed configuration at time $t$.

External virtual work at time $t+\Delta t$.

Horizon size used in the PD model.

Green Lagrange (GL) strain components at time $t+\Delta t$. These strains are measured with respect to the initial configuration.

Green Lagrange (GL) strain components at time $t$. These strains are measured with respect to the initial configuration. Incremental GL strain from time $t$ to time $t+\Delta t$. This strain is measured with respect to the initial configuration.

Nonlinear components of the incremental GL strain. This strain is measured with respect to the initial configuration.

Incremental rotations from time $t$ to time $t+\Delta t$ 


$$
\begin{aligned}
& { }^{t} \theta_{x},{ }^{t} \theta_{y} \\
& { }^{t+\Delta t} \theta_{x},{ }^{t+\Delta t} \theta_{y} \\
& { }_{0}^{t} \theta_{x, x},{ }_{0}^{t} \theta_{x, y},{ }_{0}^{t} \theta_{y, x},{ }_{0}^{t} \theta_{y, y} \\
& \underline{\mu}_{x, x},{ }_{0} \underline{\theta}_{x, y},{ }_{0} \underline{\theta}_{y, x},{ }_{0} \underline{\theta}_{y, y} \\
& { }^{v} \boldsymbol{\xi}_{(k)(j)} \\
& { }_{\rho}{ }_{{ }_{0}^{t}} \vartheta \\
& { }_{0} \underline{\vartheta} \\
& \varphi \\
& \phi \\
& { }_{0}^{t} \omega_{(k)(j)},{ }_{0}^{t} \omega_{(j)(k)} \\
& { }_{0}^{t} \omega_{(k)(j)}^{u},{ }_{0}^{t} \omega_{(k)(j)}^{v},{ }_{0}^{t} \omega_{(k)(j)}^{w}, \omega_{(k)(j)}^{\theta_{x}}, \\
& { }_{0}^{t} \omega_{(k)(j)}^{\theta_{y}}
\end{aligned}
$$

\section{Acronyms}

ADR

CCM

DOF

FEA

GL

SPK

PD
Rotations at time $t$.

Rotations at time $t+\Delta t$.

Derivatives of the rotations at time $t,{ }^{t} \theta_{x},{ }^{t} \theta_{y}$, with respect to the coordinates ${ }^{0} x,{ }^{0} y$ in the initial configuration.

Derivatives of the incremental displacements, $\underline{\theta}_{x}, \underline{\theta}_{y}$ with respect to the coordinates ${ }^{0} x,{ }^{0} y$ in the initial configuration.

Damage parameter to represent the state of interaction between material points $k$ and $j$.

Poisson's ratio of material.

Distance between material points $k$ and $j$ in the undeformed configuration.

Mass density.

Dilatation at time $t$.

Incremental dilatation.

The angle between the line connecting material point $k$ with material point $j$ and the ${ }^{0} x$ axis in the undeformed configuration.

Damage index.

Micropotentials of the interaction between material point $k$ and $j$.

Micropotential components at time $t$ of the interaction between material point $k$ and $j$. These micropotential components correspond to degrees of freedom, $u, v, w, \theta_{x}$, $\theta_{y}$, respectively.

Adaptive dynamic relaxation.

Classical continuum mechanics.

Degree of freedom (or degrees of freedom).

Finite element analysis.

Green Lagrange strain.

Second Piola-Kirchhoff stress.

Peridynamics. 


\section{Introduction}

Geometrically nonlinear analysis can be used to investigate large deformations of structures without material nonlinearity. In finite element theories, the nonlinear equation of motion (EOM) is obtained from the linearization of the principle of virtual displacements [1-3]. However, since the equations of motion in classical continuum mechanics are written in the partial-differential forms that are invalid in the presence of discontinuities, the classical continuum mechanics faces difficulties in terms of predicting complex damage patterns such as crack branching, multiple crack paths.

Peridynamics (PD) is the reformulation of continuum mechanics which is first introduced by Silling [4] and later by Silling, et al. [5], Silling and Lehoucq [6]. As opposed to classical continuum mechanics, peridynamics represents the material behaviors by using integrodifferential equations, which exists in both continuous and discontinuous models [4-7]. Therefore, discontinuities can be naturally involved in the PD analysis without any special treatment [7-13]. So far, the PD theory has been implemented in many different fields such as nonlinear elastic or plastic material responses [14-18], multiphysics and multiscale modeling [19-31], orthotropic and composite materials [32-35], and fatigue cracking [36-38]. Moreover, combining PD and FEA or implementing PD in the FEA framework are also common topics that attract many researchers [23, 32, 39-46].

Peridynamic theory includes bond-based, ordinary state-based, and nonordinary state-based models [4-8, 47]. In the bond-based PD model, since the volumetric and the deviatoric components of strain energy density are not distinguished [6-8], it has limitations in terms of Poisson's ratio of materials. Particularly, for 2D structures in the plane stress condition, the bond-based PD model can be used for material with Poisson's ratio of $1 / 3$. Meanwhile, for 2D structures in the plane strain condition, the bond-based PD model is applicable for material with Poisson's ratio of 1/4. For 3D structures, the bond-based PD model is applicable for materials with Poisson's ratio of $1 / 4$ [48].

Unlike the bond-based PD model, the ordinary state-based and nonordinary state-based PD models are applicable for materials with any Poisson's ratio. In the ordinary state-based PD model, the volumetric and the deviatoric components of strain energy density are distinguished $[5,8,48,49]$ and the definition of the force-vector state, which maps a deformation-state into force-state, is introduced [5]. In contrast to the ordinary state-based PD model, the nonordinary state-based PD model represents the force-vector state in terms of strain and stress tensors. Therefore, it allows for the incorporation of classical constitutive models into PD without the need for the reformulation of the constitutive laws in terms of the force-vector state [47].

In terms of structural simplifications for slender structures, the first simplified one-dimensional (1D) PD model to capture axial deformations for 1D bars was introduced by Silling et al. [50]. Later, O'Grady and Foster [51], Diyaroglu et al. [52, 53] introduced PD models to capture bending and transverse shear deformations for beams. Recently, Nguyen and Oterkus [54] developed a PD model to predict the damages in complex three-dimensional (3D) beam structures.

For thin-wall structures, the first simplified two-dimensional (2D) PD model was introduced by Silling and Bobaru [55], and later by Madenci and Oterkus [8]. Later, O'Grady and Foster [56] introduced a nonordinary state-based PD model to capture bending deformations of plates based on Kirchhoff-Love theory. Diyaroglu et al. [53] introduced a bond-based PD model to capture bending and transverse shear deformations of plates based on Mindlin-Reissner theory. Recently, Nguyen and Oterkus [27] developed an ordinary state-based PD model for 3D shell structures which can be used to predict damages on stiffened structures [57]. Dorduncu [58] 
and Dorduncu, et al. [59] analysed sandwich plates and laminated composite plates by using the nonlocal peridynamic differential operator $[60,61]$.

As it can be observed that the existing PD models presented in $[27,53,56,57]$ are based on the small deformation assumption in the linear analysis. It is also very common to observe large displacements, large rotations, but small elastic strains in thin-wall and slender structures. Therefore, this study proposes a novel ordinary state-based PD model for geometrically nonlinear analysis of plates based on Mindlin-Reissner theory. The nonlinear PD formulations and equations of motion are obtained based on the principle of virtual displacements by using the Total Lagrange formulation. The energy-based criterion for damage prediction is used and the numerical procedure in PD nonlinear analysis is also provided. To verify the accuracy of the proposed nonlinear PD model, large deformations of a plate subjected to bending and a plate subjected to vertical shear force are investigated. For verification purposes, the predicted results by using the proposed nonlinear PD model are compared with the nonlinear FEA results. Furthermore, damages on a plate subjected to out-of-plane stretching and tearing, a plate subjected to tearing, and a plated subjected to torsional loading are also predicted by using the nonlinear PD model.

This paper is organized as follows. Section 2 presents the principle of virtual displacement in classical continuum mechanics (CCM). Section 3 presents the nonlinear kinematics of a plate in classical continuum mechanics. Section 4 presents nonlinear kinematics of a plate in peridynamics. Section 5 presents the energy-based criterion for damage prediction. Section 6 presents the numerical procedure. The numerical results are presented in Section 7 and the conclusion is given in Section 8.

\section{Deformation gradient, Green-Lagrange strain, Second Piola-Kirchhoff stress tensors, and the principle of virtual displacement}

In this section, classical formulations for deformation gradient, Green-Lagrange strain, Second Piola-Kirchhoff stress tensors, and the principle of virtual displacement are presented. The motions of a structure at different times in the Cartesian coordinate system are shown in Fig. 1. As shown in Fig. 1, $P$ is a material point on the structure, and its coordinates at time $t=0$ are denoted by $\left({ }^{0} x,{ }^{0} y,{ }^{0} z\right)$. Meanwhile, the coordinates of this point at time $t$ and $t+\Delta t$ are denoted by $\left({ }^{t} x,{ }^{t} y,{ }^{t} z\right)$ and $\left({ }^{t+\Delta t} x,{ }^{t+\Delta t} y,{ }^{t+\Delta t} z\right)$, respectively. The relationships between these coordinates can be represented as

$$
\begin{aligned}
& { }^{t} \mathbf{X}={ }^{0} \mathbf{X}+{ }^{t} \hat{\mathbf{u}} \\
& { }^{t+\Delta t} \mathbf{X}={ }^{0} \mathbf{X}+{ }^{t+\Delta t} \hat{\mathbf{u}}
\end{aligned}
$$

with

$$
\begin{aligned}
& { }^{0} \mathbf{x}=\left[\begin{array}{lll}
{ }^{0} x & { }^{0} y & { }^{0} z
\end{array}\right]^{T} \\
& { }^{t} \mathbf{x}=\left[\begin{array}{lll}
{ }^{t} x & { }^{t} y & { }^{t} z
\end{array}\right]^{T} \\
& { }^{t+\Delta t} \mathbf{x}=\left[\begin{array}{lll}
{ }^{t+\Delta t} x & { }^{t+\Delta t} y & { }^{t+\Delta t} z
\end{array}\right]^{T} \\
& { }^{t} \hat{\mathbf{u}}=\left[\begin{array}{lll}
{ }^{t} \hat{u} & { }^{t} \hat{v} & { }^{t} \hat{w}
\end{array}\right]^{T}
\end{aligned}
$$


${ }^{t+\Delta t} \hat{\mathbf{u}}=\left[\begin{array}{lll}{ }^{t+\Delta t} \hat{u} & { }^{t+\Delta t} \hat{v} & { }^{t+\Delta t} \hat{w}\end{array}\right]^{T}$

where ${ }^{t} \hat{\mathbf{u}}$ and ${ }^{t+\Delta t} \hat{\mathbf{u}}$ represent the displacement vectors for a material point which is initially located at $\left({ }^{0} x,{ }^{0} y,{ }^{0} z\right)$ as shown in Fig. 1 at time $t$ and $t+\Delta t$, respectively. The relationship between these vectors of displacements can be represented as

${ }^{t+\Delta t} \hat{\mathbf{u}}={ }^{t} \hat{\mathbf{u}}+\underline{\hat{\mathbf{u}}}$

with

$\underline{\hat{\mathbf{u}}}=\left[\begin{array}{lll}\underline{\hat{u}} & \underline{\hat{v}} & \underline{\hat{w}}\end{array}\right]$

where $\underline{\hat{\mathbf{u}}}$ represents the vector of the incremental displacements from time $t$ to $t+\Delta t$ of a material point which is initially located at $\left({ }^{0} x,{ }^{0} y,{ }^{0} z\right)$.

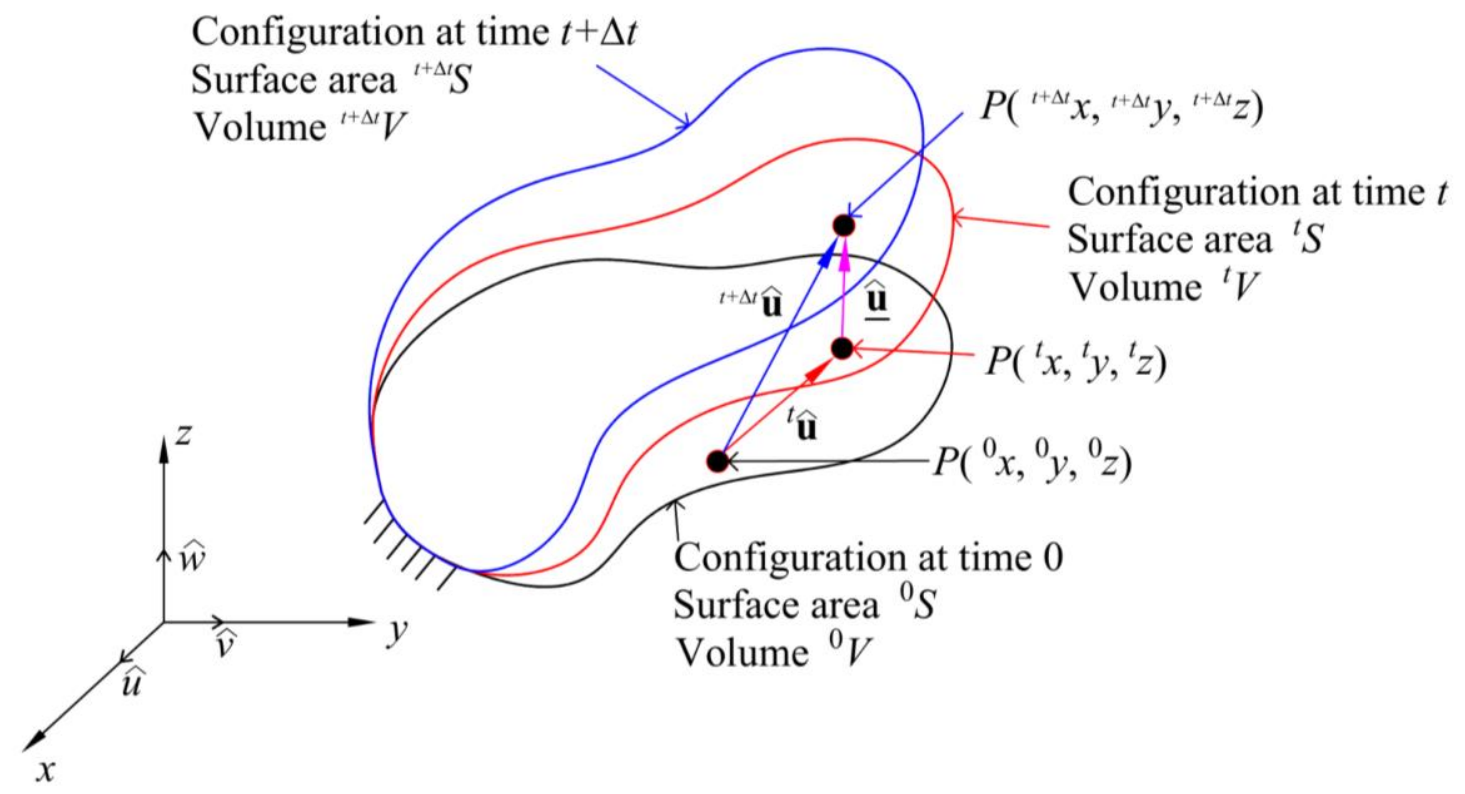

Fig. 1. Motions of a structure in the Cartesian coordinate frame

\subsection{Deformation gradient}

The deformation gradient can be defined as [1]

${ }_{0}^{t} \mathbf{X}=\left[\begin{array}{lll}\frac{\partial^{t} x}{\partial^{0} x} & \frac{\partial^{t} x}{\partial^{0} y} & \frac{\partial^{t} x}{\partial^{0} z} \\ \frac{\partial^{t} y}{\partial^{0} x} & \frac{\partial^{t} y}{\partial^{0} y} & \frac{\partial^{t} y}{\partial^{0} z} \\ \frac{\partial^{t} z}{\partial^{0} x} & \frac{\partial^{t} z}{\partial^{0} y} & \frac{\partial^{t} z}{\partial^{0} z}\end{array}\right]$

or

$$
{ }_{0}^{t} \mathbf{X}=\left({ }_{0} \nabla\left({ }^{t} \mathbf{x}^{T}\right)\right)^{T}
$$


with

$$
{ }_{0} \nabla=\left[\begin{array}{lll}
\frac{\partial}{\partial^{0} x} & \frac{\partial}{\partial^{0} y} & \frac{\partial}{\partial^{0} z}
\end{array}\right]^{T}
$$

where ${ }_{0} \nabla$ represents the gradient operator with respect to the initial configuration and ${ }^{t} \mathbf{x}^{T}$ represents the transposed vector of ${ }^{t} \mathbf{x}$.

\subsection{Green-Lagrange strain}

By using the deformation gradient given in Eq. (3), the Green-Lagrange strain at time $t$ can be defined as [1]

$$
{ }_{0}^{t} \hat{\boldsymbol{\varepsilon}}=\frac{1}{2}\left({ }_{0}^{t} \mathbf{X}^{T}{ }_{0}^{t} \mathbf{X}-\mathbf{I}\right)
$$

which can also be explicitly written as [1]

$$
\begin{aligned}
& { }_{0}^{t} \hat{\varepsilon}_{x x}={ }_{0}^{t} \hat{u}_{, x}+\frac{1}{2}\left[\left({ }_{0}^{t} \hat{u}_{, x}\right)^{2}+\left({ }_{0}^{t} \hat{v}_{, x}\right)^{2}+\left({ }_{0}^{t} \hat{w}_{, x}\right)^{2}\right] \\
& { }_{0}^{t} \hat{\varepsilon}_{y y}={ }_{0}^{t} \hat{v}_{, y}+\frac{1}{2}\left[\left({ }_{0}^{t} \hat{u}_{, y}\right)^{2}+\left({ }_{0}^{t} \hat{v}_{, y}\right)^{2}+\left({ }_{0}^{t} \hat{w}_{, y}\right)^{2}\right] \\
& { }_{0}^{t} \hat{\varepsilon}_{z z}={ }_{0}^{t} \hat{w}_{, z}+\frac{1}{2}\left[\left({ }_{0}^{t} \hat{u}_{, z}\right)^{2}+\left({ }_{0}^{t} \hat{v}_{, z}\right)^{2}+\left({ }_{0}^{t} \hat{w}_{, z}\right)^{2}\right] \\
& { }_{0}^{t} \hat{\varepsilon}_{x y}=\frac{1}{2}\left[{ }_{0}^{t} \hat{u}_{, y}+{ }_{0}^{t} \hat{v}_{, x}\right]+\frac{1}{2}\left[\left({ }_{0}^{t} \hat{u}_{, x}\right)\left({ }_{0}^{t} \hat{u}_{, y}\right)+\left({ }_{0}^{t} \hat{v}_{, x}\right)\left({ }_{0}^{t} \hat{v}_{, y}\right)+\left({ }_{0}^{t} \hat{w}_{, x}\right)\left({ }_{0}^{t} \hat{w}_{, y}\right)\right] \\
& { }_{0}^{t} \hat{\varepsilon}_{x z}=\frac{1}{2}\left[{ }_{0}^{t} \hat{u}_{, z}+{ }_{0}^{t} \hat{w}_{, x}\right]+\frac{1}{2}\left[\left({ }_{0}^{t} \hat{u}_{, x}\right)\left({ }_{0}^{t} \hat{u}_{, z}\right)+\left({ }_{0}^{t} \hat{v}_{, x}\right)\left({ }_{0}^{t} \hat{v}_{, z}\right)+\left({ }_{0}^{t} \hat{w}_{, x}\right)\left({ }_{0}^{t} \hat{w}_{, z}\right)\right] \\
& { }_{0}^{t} \hat{\varepsilon}_{y z}=\frac{1}{2}\left[{ }_{0}^{t} \hat{v}_{, z}+{ }_{0}^{t} \hat{w}_{, y}\right]+\frac{1}{2}\left[\left({ }_{0}^{t} \hat{u}_{, y}\right)\left({ }_{0}^{t} \hat{u}_{, z}\right)+\left({ }_{0}^{t} \hat{v}_{, y}\right)\left({ }_{0}^{t} \hat{v}_{, z}\right)+\left({ }_{0}^{t} \hat{w}_{, y}\right)\left({ }_{0}^{t} \hat{w}_{, z}\right)\right]
\end{aligned}
$$

where the parameter $\mathbf{I}$ in Eq. (4a) represents the identity matrix. The parameters ${ }_{0}^{t} \hat{u}_{, x},{ }_{0}^{t} \hat{u}_{, y}$, ${ }_{0}^{t} \hat{u}_{, z}$ are the derivatives of the displacement ${ }^{t} \hat{u}$ with respect to the initial configuration, ${ }^{0} x,{ }^{0} y$ , ${ }^{0} z$, respectively. The parameters ${ }_{0}^{t} \hat{v}_{, x},{ }_{0}^{t} \hat{v}_{, y},{ }_{0}^{t} \hat{v}_{, z}$ are the derivatives of the displacement ${ }^{t} \hat{v}$ with respect to the initial configuration, ${ }^{0} x,{ }^{0} y,{ }^{0} z$, respectively. Similarly, The parameters ${ }_{0}^{t} \hat{w}_{, x},{ }_{0}^{t} \hat{w}_{, y},{ }_{0}^{t} \hat{w}_{, z}$ are the derivatives of the displacement ${ }^{t} \hat{w}$ with respect to the initial configuration, ${ }^{0} x,{ }^{0} y,{ }^{0} z$, respectively. These derivatives can be represented as

$$
\begin{aligned}
& { }_{0}^{t} \hat{u}_{, x}=\frac{\partial\left({ }^{t} \hat{u}\right)}{\partial\left({ }^{0} x\right)},{ }_{0}^{t} \hat{u}_{, y}=\frac{\partial\left({ }^{t} \hat{u}\right)}{\partial\left({ }^{0} y\right)},{ }_{0}^{t} \hat{u}_{, z}=\frac{\partial\left({ }^{t} \hat{u}\right)}{\partial\left({ }^{0} z\right)} \\
& { }_{0}^{t} \hat{v}_{, x}=\frac{\partial\left({ }^{t} \hat{v}\right)}{\partial\left({ }^{0} x\right)},{ }_{0}^{t} \hat{v}_{, y}=\frac{\partial\left({ }^{t} \hat{v}\right)}{\partial\left({ }^{0} y\right)},{ }_{0}^{t} \hat{v}_{, z}=\frac{\partial\left({ }^{t} \hat{v}\right)}{\partial\left({ }^{0} z\right)}
\end{aligned}
$$




$$
{ }_{0}^{t} \hat{w}_{, x}=\frac{\partial\left({ }^{t} \hat{w}\right)}{\partial\left({ }^{0} x\right)},{ }_{0}^{t} \hat{w}_{, y}=\frac{\partial\left({ }^{t} \hat{w}\right)}{\partial\left({ }^{0} y\right)},{ }_{0}^{t} \hat{w}_{, z}=\frac{\partial\left({ }^{t} \hat{w}\right)}{\partial\left({ }^{0} z\right)}
$$

The Green-Lagrange strain at time $t+\Delta t$ can be calculated as $[1,3]$

$$
\begin{aligned}
& { }_{0}^{t+\Delta t} \hat{\varepsilon}_{x x}={ }_{0}^{t} \hat{\varepsilon}_{x x}+{ }_{0} \hat{\varepsilon}_{x x} \\
& { }^{t+\Delta t} \hat{\varepsilon}_{y y}={ }_{0}{ }_{0} \hat{\varepsilon}_{y y}+{ }_{0} \hat{\varepsilon}_{y y} \\
& { }^{t+\Delta t} \hat{\varepsilon}_{z z}={ }_{0}^{t} \hat{\varepsilon}_{z z}+{ }_{0} \hat{\varepsilon}_{z z} \\
& { }_{0}^{t+\Delta t} \hat{\varepsilon}_{x y}={ }_{0}^{t} \hat{\varepsilon}_{x y}+{ }_{0} \hat{\varepsilon}_{x y} \\
& { }^{t+\Delta t} \hat{\varepsilon}_{x z}={ }_{0}^{t} \hat{\varepsilon}_{x z}+{ }_{0} \underline{\hat{\varepsilon}}_{x z} \\
& { }^{t+\Delta t} \hat{\varepsilon}_{y z}={ }_{0}^{t} \hat{\varepsilon}_{y z}+{ }_{0} \hat{\varepsilon}_{y z}
\end{aligned}
$$

where ${ }_{0}^{t+\Delta t} \hat{\varepsilon}_{x x},{ }_{0}^{t+\Delta t} \hat{\varepsilon}_{y y},{ }_{0}^{t+\Delta t} \hat{\varepsilon}_{z z},{ }_{0}^{t+\Delta t} \hat{\varepsilon}_{x y},{ }_{0}^{t+\Delta t} \hat{\varepsilon}_{x z},{ }_{0}^{t+\Delta t} \hat{\varepsilon}_{y z}$ represent the Green-Lagrange strain components at time $t+\Delta t$. The parameters ${ }_{0}^{t} \hat{\varepsilon}_{x x},{ }_{0}^{t} \hat{\varepsilon}_{y y},{ }_{0}^{t} \hat{\varepsilon}_{z z},{ }_{0}^{t} \hat{\varepsilon}_{x y},{ }_{0}^{t} \hat{\varepsilon}_{x z},{ }_{0}^{t} \hat{\varepsilon}_{y z}$ are the GreenLagrange strain components at time $t$ as provided in Eq. (4). The parameters ${ }_{0} \underline{\hat{\varepsilon}}_{x x},{ }_{0} \underline{\hat{\varepsilon}}_{y y},{ }_{0} \hat{\varepsilon}_{z z}$ ${ }_{0} \underline{\hat{\varepsilon}}_{x y},{ }_{0} \hat{\hat{\varepsilon}}_{x z},{ }_{0} \underline{\hat{\varepsilon}}_{y z}$ represent the incremental Green-Lagrange strain from time $t$ to time $t+\Delta t$ . These incremental strain components can be decomposed as [1]

$$
\begin{aligned}
& { }_{0} \hat{\underline{\varepsilon}}_{x x}={ }_{0} \underline{\hat{\boldsymbol{e}}}_{x x}+{ }_{0} \underline{\hat{\eta}}_{x x} \\
& { }_{0} \underline{\hat{\varepsilon}}_{y y}={ }_{0} \underline{\hat{\theta}}_{y y}+{ }_{0} \underline{\hat{\eta}}_{y y} \\
& { }_{0} \underline{\hat{\boldsymbol{\varepsilon}}}_{z z}={ }_{0} \underline{\hat{\boldsymbol{e}}}_{z z}+{ }_{0} \underline{\hat{\eta}}_{z z} \\
& { }_{0} \hat{\underline{\varepsilon}}_{x y}={ }_{0} \underline{\hat{\boldsymbol{e}}}_{x y}+{ }_{0} \underline{\hat{\eta}}_{x y} \\
& { }_{0} \hat{\boldsymbol{\varepsilon}}_{x z}={ }_{0} \underline{\hat{\boldsymbol{e}}}_{x z}+{ }_{0} \underline{\hat{\eta}}_{x z} \\
& { }_{0} \underline{\hat{\varepsilon}}_{y z}={ }_{0} \underline{\hat{\boldsymbol{e}}}_{y z}+{ }_{0} \underline{\hat{\eta}}_{y z}
\end{aligned}
$$

with

$$
\begin{aligned}
& { }_{0} \underline{\hat{e}}_{x x}={ }_{0} \underline{\hat{u}}_{, x}+{ }_{0}^{t} \hat{u}_{, x 0} \underline{\hat{u}}_{, x}+{ }_{0}^{t} \hat{v}_{, x} 0 \underline{\hat{v}}_{, x}+{ }_{0}^{t} \hat{w}_{, x} 0 \underline{\hat{w}}_{, x} \\
& { }_{0} \underline{\hat{e}}_{y y}={ }_{0} \underline{\hat{v}}_{, y}+{ }_{0}^{t} \hat{v}_{, y 0} \underline{\hat{v}}_{, y}+{ }_{0}^{t} \hat{u}_{, y} \underline{\hat{u}}_{, y}+{ }_{0}^{t} \hat{w}_{, y 0} \hat{\underline{w}}_{, y} \\
& { }_{0} \underline{\hat{e}}_{z z}={ }_{0} \underline{\hat{w}}_{, z}+{ }_{0}^{t} \hat{u}_{, z 0} \underline{\hat{u}}_{, z}+{ }_{0}^{t} \hat{v}_{, z 0} \underline{\hat{\hat{v}}}_{z}+{ }_{0}^{t} \hat{w}_{, z 0} \underline{\hat{w}}_{, z} \\
& { }_{0} \underline{\hat{e}}_{x y}=\frac{1}{2}\left({ }_{0} \underline{\hat{u}}_{, y}+{ }_{0} \underline{\hat{v}}_{, x}\right)+\frac{1}{2}\left(\begin{array}{l}
{ }_{0}^{t} \hat{u}_{, x 0} \hat{\hat{u}}_{, y}+{ }_{0}^{t} \hat{u}_{, y 0} \underline{\hat{u}}_{, x}+{ }_{0}^{t} \hat{v}_{, x 0} \hat{\hat{v}}_{, y}+{ }_{0}^{t} \hat{v}_{, y 0} \underline{\hat{v}}_{, x} \\
+{ }_{0}^{t} \hat{w}_{, x 0} \underline{\hat{w}}_{, y}+{ }_{0}^{t} \hat{w}_{, y 0} \underline{\hat{w}}_{, x}
\end{array}\right) \\
& { }_{0} \underline{\hat{e}}_{x z}=\frac{1}{2}\left({ }_{0} \underline{\hat{u}}_{, z}+{ }_{0} \underline{\hat{w}}_{, x}\right)+\frac{1}{2}\left(\begin{array}{l}
{ }_{0}^{t} \hat{u}_{, x 0} \hat{\hat{u}}_{, z}+{ }_{0}^{t} \hat{u}_{, z 0} \underline{\hat{u}}_{, x}+{ }_{0}^{t} \hat{v}_{, x 0} \underline{\hat{v}}_{, z}+{ }_{0}^{t} \hat{v}_{, z 0} \hat{\underline{v}}_{, x} \\
+{ }_{0}^{t} \hat{w}_{, x 0} \underline{\hat{w}}_{, z}+{ }_{0}^{t} \hat{w}_{, z 0} \underline{\hat{w}}_{, x}
\end{array}\right)
\end{aligned}
$$




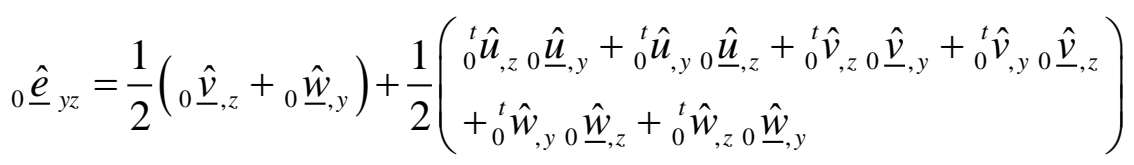

and

$$
\begin{aligned}
& { }_{0} \underline{\hat{\underline{\hat{p}}}}_{x x}=\frac{1}{2}\left(\left(_{0} \underline{\hat{\hat{u}}}_{, x}\right)^{2}+\left({ }_{0} \underline{\hat{\hat{v}}}_{, x}\right)^{2}+\left({ }_{0} \hat{\underline{\hat{w}}}_{, x}\right)^{2}\right) \\
& { }_{0} \underline{\hat{\underline{\eta}}}_{y y}=\frac{1}{2}\left(\left({ }_{0} \underline{\hat{u}}_{, y}\right)^{2}+\left({ }_{0} \underline{\hat{\hat{v}}}_{, y}\right)^{2}+\left({ }_{0} \underline{\hat{\hat{w}}}_{y}\right)^{2}\right) \\
& { }_{0} \underline{\hat{\underline{\eta}}}_{z z}=\frac{1}{2}\left(\left({ }_{0} \underline{\hat{u}}_{, z}\right)^{2}+\left({ }_{0} \underline{\hat{\hat{v}}}_{, z}\right)^{2}+\left({ }_{0} \underline{\hat{\underline{w}}}_{, z}\right)^{2}\right) \\
& { }_{0} \underline{\hat{\eta}}_{x y}=\frac{1}{2}\left({ }_{0} \underline{\hat{u}}_{, x 0} \underline{\hat{u}}_{, y}+{ }_{0} \underline{\hat{v}}_{, x 0} \underline{\hat{v}}, y_{, y}+{ }_{0} \underline{\hat{w}}_{, x 0} \underline{\hat{w}}_{, y}\right) \\
& { }_{0} \underline{\hat{\underline{\eta}}}_{x z}=\frac{1}{2}\left({ }_{0} \underline{\hat{u}}_{, x 0} \underline{\hat{u}}_{, z}+{ }_{0} \hat{\hat{v}}_{, x 0} \underline{\hat{v}}_{, z}+{ }_{0} \underline{\hat{w}}_{, x 0} \underline{\hat{w}}_{, z}\right) \\
& { }_{0} \underline{\hat{\eta}}_{y z}=\frac{1}{2}\left({ }_{0} \underline{\hat{u}}_{, y} \underline{\hat{u}}_{, z}+{ }_{0} \underline{\hat{v}}_{, y} 0 \underline{\hat{v}}_{, z}+{ }_{0} \underline{\hat{w}}_{, y} \underline{\hat{w}}_{, z}\right)
\end{aligned}
$$

where ${ }_{0} \underline{\hat{e}}_{x x},{ }_{0} \underline{\hat{e}}_{y y},{ }_{0} \hat{\hat{e}}_{z z},{ }_{0} \underline{\hat{e}}_{x y},{ }_{0} \hat{\hat{e}}_{x z},{ }_{0} \underline{\hat{e}}_{y z}$ represent the linear components of the incremental Green-Lagrange strains. The parameters ${ }_{0} \underline{\hat{\eta}}_{x x},{ }_{0} \hat{\hat{\eta}}_{y y},{ }_{0} \hat{\hat{\eta}}_{z z},{ }_{0} \underline{\hat{\eta}}_{x y},{ }_{0} \underline{\hat{\eta}}_{x z},{ }_{0} \hat{\hat{\eta}}_{y z}$ represent the nonlinear components of the incremental Green-Lagrange strains.

In Eqs. (8-9), the terms ${ }_{0} \underline{\hat{u}}_{, x},{ }_{0} \underline{\hat{u}}_{, y},{ }_{0} \underline{\hat{u}}_{, z}$ represent the derivatives of the incremental displacement $\underline{\hat{u}}$ with respect to the initial configuration, ${ }^{0} x,{ }^{0} y,{ }^{0} z$, respectively. Similarly, the terms ${ }_{0} \hat{\hat{v}}_{, x},{ }_{0} \hat{\hat{v}}_{y},{ }_{0} \hat{\hat{v}}_{, z}$ represent the derivatives of the incremental displacement $\underline{\hat{v}}$ with respect to the initial configuration, ${ }^{0} x,{ }^{0} y,{ }^{0} z$, respectively. The terms ${ }_{0} \underline{\hat{w}}_{, x},{ }_{0} \underline{\hat{w}}_{, y},{ }_{0} \underline{\hat{w}}_{, z}$ represent the derivatives of the incremental displacement $\underline{\hat{w}}$ with respect to the initial configuration, ${ }^{0} x,{ }^{0} y,{ }^{0} z$, respectively. These derivatives can be defined as [1]

$$
\begin{aligned}
& { }_{0} \underline{\hat{u}}_{, x}=\frac{\partial(\underline{\hat{u}})}{\partial\left({ }^{0} x\right)},{ }_{0} \hat{\hat{u}}_{, y}=\frac{\partial(\underline{\hat{u}})}{\partial\left({ }^{0} y\right)},{ }_{0} \hat{\underline{u}}_{, z}=\frac{\partial(\underline{\hat{u}})}{\partial\left({ }^{0} z\right)} \\
& { }_{0} \underline{\hat{v}}_{x}=\frac{\partial(\underline{\hat{v}})}{\partial\left({ }^{0} x\right)}, \quad{ }_{0} \underline{\hat{v}}_{y}=\frac{\partial(\underline{\hat{v}})}{\partial\left({ }^{0} y\right)},{ }_{0} \hat{\hat{v}}_{z}=\frac{\partial(\underline{\hat{v}})}{\partial\left({ }^{0} z\right)} \\
& { }_{0} \hat{\hat{w}}_{, x}=\frac{\partial(\underline{\hat{w}})}{\partial\left({ }^{0} x\right)},{ }_{0} \hat{\hat{w}}_{, y}=\frac{\partial(\underline{\hat{w}})}{\partial\left({ }^{0} y\right)},{ }_{0} \hat{\underline{\hat{w}}}_{, z}=\frac{\partial(\underline{\hat{w}})}{\partial\left({ }^{0} z\right)}
\end{aligned}
$$

Note that, the derivatives ${ }_{0}^{t} \hat{u}_{, x},{ }_{0}^{t} \hat{u}_{y, y},{ }_{0}^{t} \hat{u}_{, z},{ }_{0}^{t} \hat{v}_{, x},{ }_{0}^{t} \hat{v}_{, y},{ }_{0}^{t} \hat{v}_{, z},{ }_{0}^{t} \hat{w}_{, x},{ }_{0}^{t} \hat{w}_{, y},{ }_{0}^{t} \hat{w}_{, z}$ in Eq. (5) has left superscript " $t$ " because they are the derivatives of the displacements $\left({ }^{t} \hat{u},{ }^{t} \hat{v},{ }^{t} \hat{w}\right)$ at time $t$. On the other hand, the derivatives ${ }_{0} \underline{\hat{u}}_{, x},{ }_{0} \underline{\hat{u}}_{, y},{ }_{0} \hat{\underline{u}}_{, z},{ }_{0} \hat{\hat{v}}_{, x},{ }_{0} \underline{\hat{v}}_{, y},{ }_{0} \hat{\underline{\hat{v}}}_{, z},{ }_{0} \underline{\hat{\hat{w}}}_{, x},{ }_{0} \hat{\underline{\hat{w}}}_{, y},{ }_{0} \underline{\hat{\hat{w}}}_{, z}$ in Eq. (10) do not have any left superscript since they are the derivatives of the incremental 
displacements $(\underline{\hat{u}}, \underline{\hat{v}}, \underline{\hat{w}})$. The demonstration of derivatives ${ }_{0}^{t} \hat{u}_{, x}$ and ${ }_{0} \underline{\hat{u}}_{, x}$ are shown in Fig. 2 .

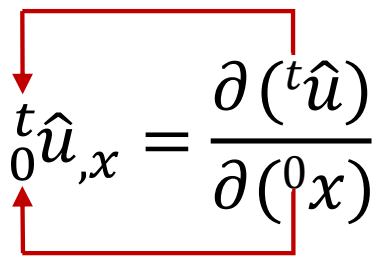

(a)

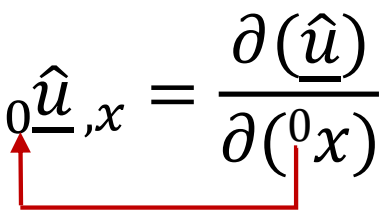

(b)

Fig. 2. Demonstration of derivatives (a): derivative of displacement at time $t$ with respect to the initial configuration, (b) derivative of incremental displacement from time $t$ to $t+\Delta t$ with respect to the initial configuration.

In Fig. 2, the left superscript " $t$ " represents the current time. Meanwhile, the left subscript " 0 " represents that the derivatives are calculated with respect to the initial configuration.

\subsection{Second Piola-Kirchhoff stress}

In nonlinear analyses, the second Piola-Kirchhoff stress tensor is always work-conjugate with the Green-Lagrange tensor since they are often used as a pair [1]. For plates with large deformations but small elastic strain problems, the through-thickness stress component is zero ( ${ }_{0}^{t} \hat{S}_{z z}=0$ ) since the plane stress condition is considered. Therefore, the second Piola-Kirchhoff stress tensor can be simply estimated as [1]

${ }_{0}^{t} \hat{\mathbf{S}}={ }_{0}^{t} \mathbf{C}_{0}^{t} \hat{\boldsymbol{\varepsilon}}$

with

${ }_{0}^{t} \hat{\mathbf{S}}=\left[{ }_{0}^{t} \hat{S}_{x x} \quad{ }_{0}^{t} \hat{S}_{y y} \quad{ }_{0}^{t} \hat{S}_{x y} \quad{ }_{0}^{t} \hat{S}_{x z} \quad{ }_{0}^{t} \hat{S}_{y z}\right]$

and

${ }_{0}^{t} \hat{\boldsymbol{\varepsilon}}=\left[\begin{array}{lllll}{ }_{0}^{t} \hat{\varepsilon}_{x x} & { }_{0}^{t} \hat{\varepsilon}_{y y} & { }_{0}^{t} \hat{\varepsilon}_{x y} & { }_{0}^{t} \hat{\varepsilon}_{x z} & { }_{0}^{t} \hat{\varepsilon}_{y z}\end{array}\right]$

where ${ }_{0}^{t} \hat{\mathbf{S}}$ represents the second Piola-Kirchhoff stress tensor. This stress tensor is measured with respect to the undeformed configuration at $t=0$. The parameter ${ }_{0}^{t} \mathbf{C}$ represents the elasticity tensor and ${ }_{0}^{t} \hat{\boldsymbol{\varepsilon}}$ represents the Green-Lagrange strain at time $t$. For plates and shells, the elasticity tensor can be represented as [1]

$$
{ }_{0}^{t} \mathbf{C}=\frac{E}{1-v^{2}}\left[\begin{array}{ccccc}
1 & v & 0 & 0 & 0 \\
v & 1 & 0 & 0 & 0 \\
0 & 0 & (1-v) & 0 & 0 \\
0 & 0 & 0 & k_{s}(1-v) & 0 \\
0 & 0 & 0 & 0 & k_{s}(1-v)
\end{array}\right]
$$

with

$k_{s}=5 / 6$ 
where $E$ and $v$ represent the elastic modulus and Poisson's ratio of the material, $k_{s}$ represents shear correction factor [1].

The second Piola-Kirchhoff stress tensor at time $t+\Delta t$ can be represented as $[1,3]$

$$
{ }_{0}^{t+\Delta t} \hat{\mathbf{S}}={ }_{0}^{t} \hat{\mathbf{S}}+{ }_{0} \hat{\mathbf{S}}
$$

or

$$
\begin{aligned}
& { }_{0}^{t+\Delta t} \hat{S}_{x x}={ }_{0}^{t} \hat{S}_{x x}+{ }_{0} \hat{S}_{x x} \\
& { }^{t+\Delta t} \hat{S}_{y y}={ }_{0}^{t} \hat{S}_{y y}+{ }_{0} \hat{S}_{y y} \\
& { }^{t+\Delta t} \hat{S}_{x y}={ }_{0}^{t} \hat{S}_{x y}+{ }_{0} \underline{S}_{x y} \\
& { }_{0}^{t+\Delta t} \hat{S}_{x z}={ }_{0}^{t} \hat{S}_{x z}+{ }_{0} \hat{S}_{x z} \\
& { }^{t+\Delta t} \hat{S}_{y z}={ }_{0}^{t} \hat{S}_{y z}+{ }_{0} \underline{S}_{y z} \\
& { }^{t+\Delta t} \hat{S}_{z z}={ }_{0}^{t} \hat{S}_{z z}=0
\end{aligned}
$$

where ${ }_{0}^{t+\Delta t} \hat{S}_{x x},{ }_{0}^{t+\Delta t} \hat{S}_{y y},{ }_{0}^{t+\Delta t} \hat{S}_{x y},{ }_{0}^{t+\Delta t} \hat{S}_{x z},{ }_{0}^{t+\Delta t} \hat{S}_{y z}$ are nonzero stress components of the stress tensor ${ }^{t+\Delta t} \hat{\mathbf{S}}$ at time $t+\Delta t$. The parameter ${ }_{0} \underline{\hat{\mathbf{S}}}$ represents the incremental second Piola-Kirchhoff stress tensor from time $t$ to time $t+\Delta t$. The terms ${ }_{0} \underline{\hat{S}}_{x x},{ }_{0} \underline{\hat{S}}_{y y},{ }_{0} \underline{\hat{S}}_{x y},{ }_{0} \underline{\hat{S}}_{x z},{ }_{0} \underline{\hat{S}}_{y z}$ are nonzero stress components of the incremental stress tensor ${ }_{0} \underline{\mathbf{S}}$.

Note that, the relation given in Eq. (11) is applicable for elastic material responses with large deformations but small strains [1]. For large elastic strain problems, the second Piola-Kirchhoff stress tensor can be calculated by using the relationship with the Cauchy stress [1]. In this study, geometrically nonlinear analyses for plates with large displacements, large rotations, but small elastic strains are considered [1]. Therefore, the relation given in Eq. (11) is used.

\subsection{Principle of virtual displacement}

In this section, the principle of virtual displacement based on Total Lagrange formulation is presented. In the Total Lagrange formulation, the equation of motion for a structure is written with respect to the initial configuration as [1]

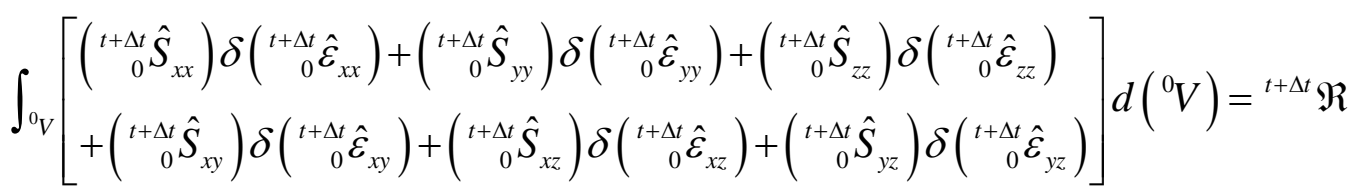

where ${ }_{0}^{t+\Delta t} \hat{S}_{x x},{ }_{0}^{t+\Delta t} \hat{S}_{y y},{ }_{0}^{t+\Delta t} \hat{S}_{z z},{ }_{0}^{t+\Delta t} \hat{S}_{x y},{ }_{0}^{t+\Delta t} \hat{S}_{x z},{ }_{0}^{t+\Delta t} \hat{S}_{y z}$ represent components of Second PiolaKirchhoff stress tensor at time $t+\Delta t$ as given in Eq. (13). The parameters ${ }_{0}^{t+\Delta t} \hat{\varepsilon}_{x x},{ }_{0}^{t+\Delta t} \hat{\varepsilon}_{y y},{ }_{0}^{t+\Delta t} \hat{\varepsilon}_{z z}$ ${ }_{0}^{t+\Delta t} \hat{\varepsilon}_{x y},{ }_{0}^{t+\Delta t} \hat{\varepsilon}_{x z},{ }_{0}^{t+\Delta t} \hat{\varepsilon}_{y z}$ represent components of Green Lagrange strain at time $t+\Delta t$ as given

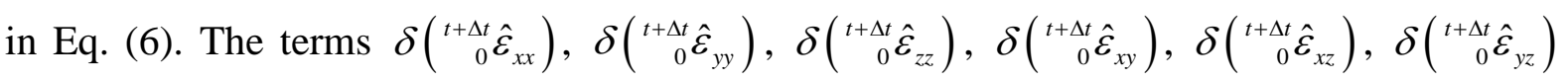
represent the virtual value of ${ }_{0}^{t+\Delta t} \hat{\varepsilon}_{x x},{ }_{0}^{t+\Delta t} \hat{\varepsilon}_{y y},{ }_{0}^{t+\Delta t} \hat{\varepsilon}_{z z},{ }_{0}^{t+\Delta t} \hat{\varepsilon}_{x y},{ }_{0}^{t+\Delta t} \hat{\varepsilon}_{x z},{ }_{0}^{t+\Delta t} \hat{\varepsilon}_{y z}$, respectively. The 
parameter ${ }^{0} V$ represents the volume in the undeformed configuration, ${ }^{t+\Delta t} \mathfrak{R}$ represents the external virtual work.

By using the relations given in Eq. (6) and Eq. (13), the principle of virtual displacements given in Eq. (14) can be rewritten as

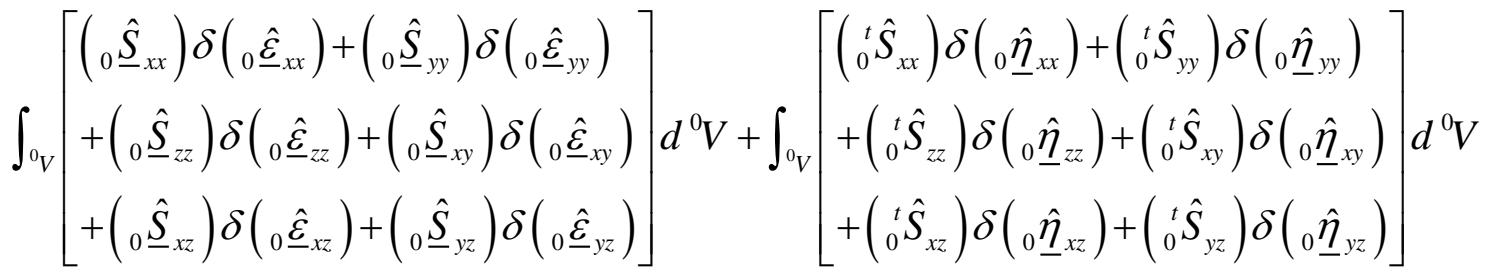

$$
\begin{aligned}
& ={ }^{t+\Delta t} \mathfrak{R}-\int_{{ }^{0} V}\left[\begin{array}{l}
\left({ }_{0}^{t} \hat{S}_{x x}\right) \delta\left({ }_{0} \underline{\hat{e}}_{x x}\right)+\left({ }_{0}^{t} \hat{S}_{y y}\right) \delta\left({ }_{0} \underline{\hat{e}}_{y y}\right)+\left({ }_{0}^{t} \hat{S}_{z z}\right) \delta\left({ }_{0} \underline{\hat{\boldsymbol{e}}}_{z z}\right) \\
+\left({ }_{0}^{t} \hat{S}_{x y}\right) \delta\left({ }_{0} \underline{\hat{\boldsymbol{e}}}_{x y}\right)+\left({ }_{0}^{t} \hat{S}_{x z}\right) \delta\left({ }_{0} \underline{\hat{\boldsymbol{e}}}_{x z}\right)+\left({ }_{0}^{t} \hat{S}_{y z}\right) \delta\left({ }_{0} \underline{\hat{\boldsymbol{e}}}_{y z}\right)
\end{array}\right] d^{0} V
\end{aligned}
$$

For dynamic problems, the work caused by the inertia forces can be added on the left-hand side of Eq. (15) as [1]

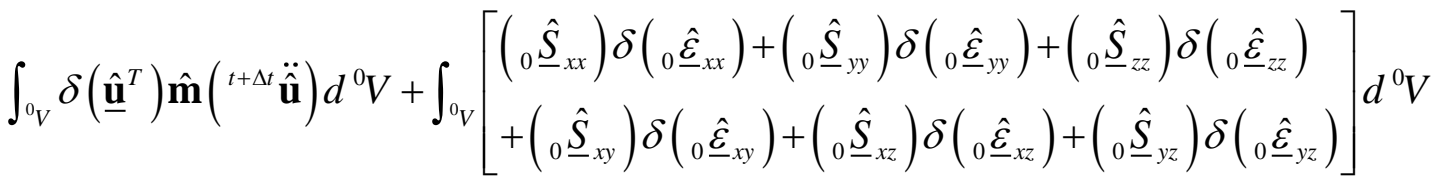

$$
\begin{aligned}
& +\int_{{ }^{0} V}\left[\begin{array}{l}
\left({ }_{0}^{t} \hat{S}_{x x}\right) \delta\left({ }_{0} \underline{\hat{\eta}}_{x x}\right)+\left({ }_{0}^{t} \hat{S}_{y y}\right) \delta\left({ }_{0} \underline{\hat{\eta}}_{y y}\right)+\left({ }_{0}^{t} \hat{S}_{z z}\right) \delta\left({ }_{0} \underline{\hat{\eta}}_{z z}\right) \\
+\left({ }_{0}^{t} \hat{S}_{x y}\right) \delta\left({ }_{0} \underline{\hat{\eta}}_{x y}\right)+\left({ }_{0}^{t} \hat{S}_{x z}\right) \delta\left({ }_{0} \underline{\hat{\eta}}_{x z}\right)+\left({ }_{0}^{t} \hat{S}_{y z}\right) \delta\left({ }_{0} \underline{\hat{\eta}}_{y z}\right)
\end{array}\right] d^{0} V \\
& ={ }^{t+\Delta t} \mathfrak{R}-\int_{{ }^{0_{V}}}\left[\begin{array}{l}
\left({ }_{0}^{t} \hat{S}_{x x}\right) \delta\left({ }_{0} \underline{\hat{e}}_{x x}\right)+\left({ }_{0}^{t} \hat{S}_{y y}\right) \delta\left({ }_{0} \underline{\hat{e}}_{y y}\right)+\left({ }_{0}^{t} \hat{S}_{z z}\right) \delta\left({ }_{0} \underline{\hat{e}}_{z z}\right) \\
+\left({ }_{0}^{t} \hat{S}_{x y}\right) \delta\left({ }_{0} \underline{\hat{e}}_{x y}\right)+\left({ }_{0}^{t} \hat{S}_{x z}\right) \delta\left({ }_{0} \underline{\hat{e}}_{x z}\right)+\left({ }_{0}^{t} \hat{S}_{y z}\right) \delta\left({ }_{0} \underline{\hat{e}}_{y z}\right)
\end{array}\right] d^{0} V
\end{aligned}
$$

where the first term on the left-hand side of Eq. (16) represents the virtual work of the inertia forces, $\hat{\mathbf{m}}$ represents the time-independent mass matrix, ${ }^{t+\Delta t} \ddot{\hat{\mathbf{u}}}$ represents the acceleration vector at time $t+\Delta t$ of the material point located at $\left({ }^{0} x,{ }^{0} y,{ }^{0} z\right)$, $\underline{\mathbf{u}}$ represents the incremental displacement vector of the material point located at $\left({ }^{0} x,{ }^{0} y,{ }^{0} z\right)$ as given in Eq. $(2 \mathrm{~b}), \underline{\hat{\mathbf{u}}}^{T}$ represents the transpose of the incremental displacement vector, $\underline{\hat{\mathbf{u}}}$.

According to Bathe [1], the equation of motion given in Eq. (16) is written for dynamic problems for the implicit time integration scheme. If the explicit time integration scheme is used, Eq. (16) can be simplified as [1]

$$
\int_{0_{V}} \delta\left(\underline{\hat{\mathbf{u}}}^{T}\right) \hat{\mathbf{m}}\left({ }^{t} \ddot{\hat{\mathbf{u}}}\right) d^{0} V={ }^{t+\Delta t} \mathfrak{R}-\int_{0_{V}}\left[\begin{array}{l}
\left({ }_{0}^{t} \hat{S}_{x x}\right) \delta\left({ }_{0} \underline{\hat{e}}_{x x}\right)+\left({ }_{0}^{t} \hat{S}_{y y}\right) \delta\left({ }_{0} \underline{\hat{e}}_{y y}\right)+\left({ }_{0}^{t} \hat{S}_{z z}\right) \delta\left({ }_{0} \hat{\hat{e}}_{z z}\right) \\
+\left({ }_{0}^{t} \hat{S}_{x y}\right) \delta\left({ }_{0} \underline{\hat{e}}_{x y}\right)+\left({ }_{0}{ }_{0} \hat{S}_{x z}\right) \delta\left({ }_{0} \underline{\hat{e}}_{x z}\right)+\left({ }_{0}{ }_{0}^{t} \hat{S}_{y z}\right) \delta\left({ }_{0} \underline{\hat{e}}_{y z}\right)
\end{array}\right] d^{0} V
$$

The second term on the right-hand side of Eq. (17) is the virtual work which is associated with the element stress at time $t$. The corresponding strain energy density (SED) for this virtual work can be calculated as [14]

$$
W^{N L}={ }_{0}^{t} \hat{S}_{x x} 0 \underline{\hat{e}}_{x x}+{ }_{0}^{t} \hat{S}_{y y 0} \underline{\hat{e}}_{y y}+{ }_{0}^{t} \hat{S}_{z z} \underline{\hat{e}}_{z z}+2{ }_{0}^{t} \hat{S}_{x y 0} \underline{\hat{e}}_{x y}+2{ }_{0}^{t} \hat{S}_{y z 0} \underline{\hat{e}}_{y z}+2{ }_{0}^{t} \hat{S}_{x z 0} \underline{\hat{e}}_{x z}
$$


In this study, the SED in classical continuum mechanics for a plate with large displacements, large rotations, but small elastic strains, is obtained by using Eq. (18).

\section{Nonlinear kinematics of plates in Classical Continuum Mechanics}

In this section, the nonlinear kinematics of a plate are presented. According to Mindlin [62], Reissner [63], each material point on the mid-plane of the plate has five degrees of freedom, including three displacements $(u, v, w)$ and two rotations $\left(\theta_{x}, \theta_{y}\right)$ as shown in Fig. 3 and Fig. 4.

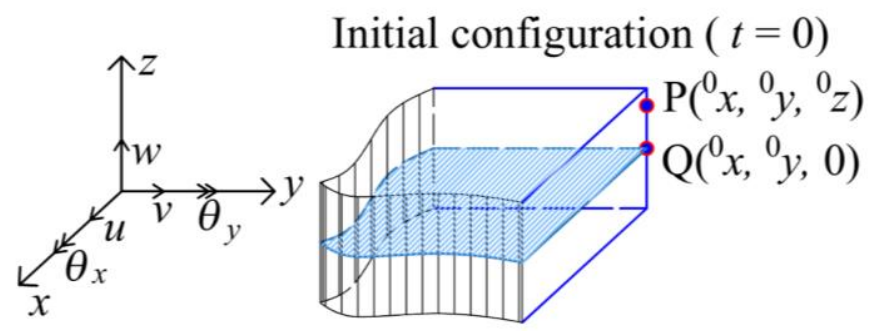

Deformed configuration at time $t$

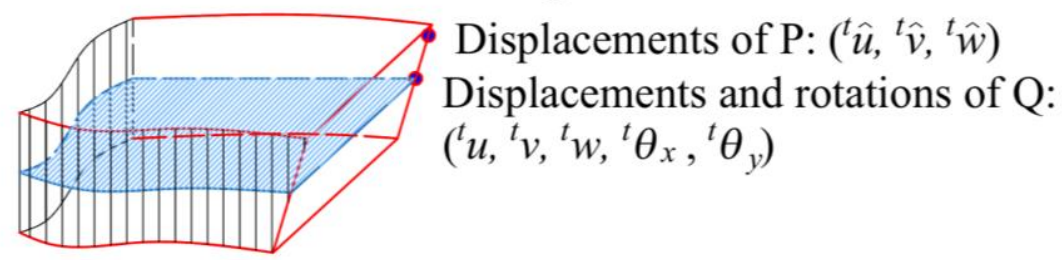

Deformed configuration at time $t+\Delta t$

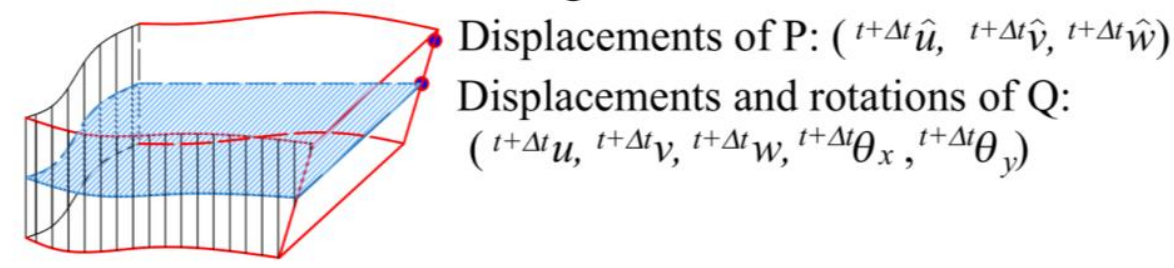

Fig. 3. Displacement vectors of a material point located on the mid-plane and a material point located at any location in the initial and deformed configurations of a plate 


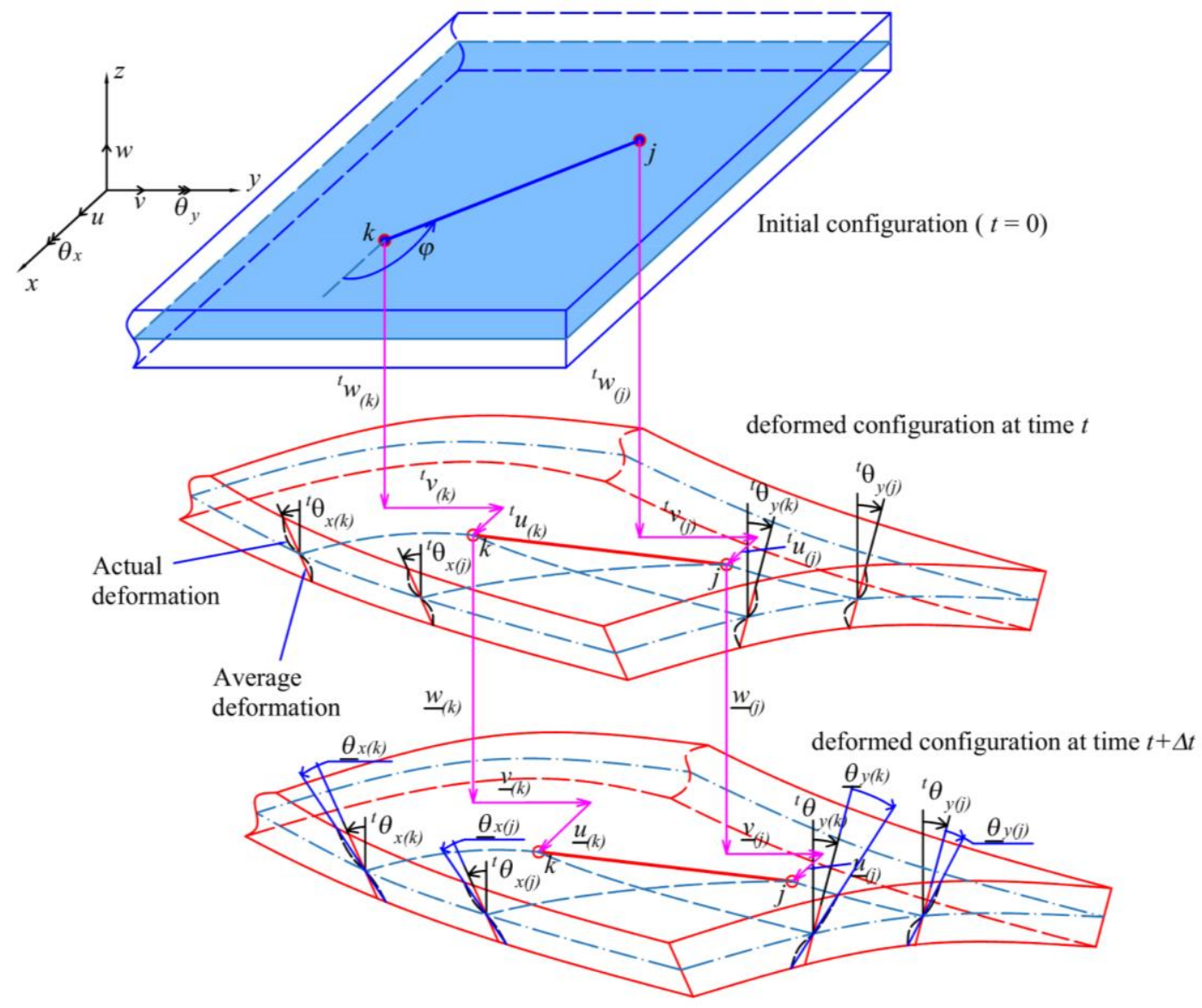

Fig. 4. Five degrees of freedom of material points located on the mid-plane in the initial and deformed configurations of a plate

\subsection{Displacement field}

As shown in Fig. 3(b), material point $P$ is located at $\left({ }^{0} x,{ }^{0} y,{ }^{0} z\right)$ and material point $Q$ is located at $\left({ }^{0} x,{ }^{0} y, 0\right)$ which is at the mid-plane of the undeformed plate. The degree of freedoms at time $t$ and time $t+\Delta t$ for material points $P$ and $Q$ are represented as

$\left({ }^{t} \hat{u},{ }^{t} \hat{v},{ }^{t} \hat{w}\right),\left({ }^{t+\Delta t} \hat{u},{ }^{t+\Delta t} \hat{v},{ }^{t+\Delta t} \hat{w}\right)$ for $P$

$\left({ }^{t} u,{ }^{t} v,{ }^{t} w,{ }^{t} \theta_{x},{ }^{t} \theta_{y}\right),\left({ }^{t+\Delta t} u,{ }^{t+\Delta t} v,{ }^{t+\Delta t} w,{ }^{t+\Delta t} \theta_{x},{ }^{t+\Delta t} \theta_{y}\right)$ for $Q$

According to Barut [64], the displacements of material point $P$ located at $\left({ }^{0} x,{ }^{0} y,{ }^{0} z\right)$ at time $t$ and $t+\Delta t$ can be represented as [64]

$$
\begin{aligned}
& { }^{t} \hat{u}={ }^{t} u+{ }^{0} z \sin \left({ }^{t} \theta_{y}\right),{ }^{t+\Delta t} \hat{u}={ }^{t+\Delta t} u+{ }^{0} z \sin \left({ }^{t+\Delta t} \theta_{y}\right) \\
& { }^{t} \hat{v}={ }^{t} v-{ }^{0} z \sin \left({ }^{t} \theta_{x}\right),{ }^{t+\Delta t} \hat{v}={ }^{t+\Delta t} v-{ }^{0} z \sin \left({ }^{t+\Delta t} \theta_{x}\right) \\
& { }^{t} \hat{w}={ }^{t} w,{ }^{t+\Delta t} \hat{w}={ }^{t+\Delta t} w
\end{aligned}
$$

with

$-h / 2 \leq{ }^{0} z \leq h / 2$ 
where $h$ represents the thickness of the plate.

On the other hand, the incremental displacements from time $t$ to time $t+\Delta t$ of material point $P$ located at $\left({ }^{0} x,{ }^{0} y,{ }^{0} z\right)$ can be calculated by using Eq. (2) as

$$
\begin{aligned}
& \underline{\hat{u}}={ }^{t+\Delta t} \hat{u}-{ }^{t} \hat{u} \\
& \underline{\hat{v}}={ }^{t+\Delta t} \hat{v}-{ }^{t} \hat{v} \\
& \underline{\hat{w}}={ }^{t+\Delta t} \hat{w}-{ }^{t} \hat{w}
\end{aligned}
$$

By using Eq. (20), the incremental displacements in Eq. (21) can be represented as

$$
\begin{aligned}
& \underline{\hat{u}}=\left({ }^{t+\Delta t} u-{ }^{t} u\right)+{ }^{0} z\left[\sin \left({ }^{t+\Delta t} \theta_{y}\right)-\sin \left({ }^{t} \theta_{y}\right)\right] \\
& \underline{\hat{v}}=\left({ }^{t+\Delta t} v-{ }^{t} v\right)-{ }^{0} z\left[\sin \left({ }^{t+\Delta t} \theta_{x}\right)-\sin \left({ }^{t} \theta_{x}\right)\right] \\
& \underline{\hat{w}}={ }^{t+\Delta t} w-{ }^{t} \mathcal{W}
\end{aligned}
$$

which can be rewritten as

$$
\begin{aligned}
& \underline{\hat{u}}=\underline{u}+{ }^{0} z\left[\sin \left({ }^{t+\Delta t} \theta_{y}\right)-\sin \left({ }^{t} \theta_{y}\right)\right] \\
& \hat{\underline{v}}=\underline{v}-{ }^{0} z\left[\sin \left({ }^{t+\Delta t} \theta_{x}\right)-\sin \left({ }^{t} \theta_{x}\right)\right] \\
& \underline{\hat{w}}=\underline{w}
\end{aligned}
$$

with

$$
\begin{aligned}
& \underline{u}={ }^{t+\Delta t} u-{ }^{t} u \\
& \underline{v}={ }^{t+\Delta t} v-{ }^{t} v \\
& \underline{w}={ }^{t+\Delta t} w-{ }^{t} w
\end{aligned}
$$

where $\underline{u}, \underline{v}, \underline{w}$ represent the incremental displacements from time $t$ to time $t+\Delta t$ of point $Q$ located at $\left({ }^{0} x,{ }^{0} y, 0\right)$ at the mid-plane of the plate.

By using the Eqs. (23a-c), the incremental displacements at any point $P$ can be rewritten in terms of incremental displacements of point $Q$ at the mid-plane by using trigonometric relations as

$$
\begin{aligned}
& \underline{\hat{u}}=\underline{u}+2{ }^{0} z \sin \left(\frac{\underline{\theta}_{y}}{2}\right)\left[\cos \left({ }^{t} \theta_{y}\right) \cos \left(\frac{\underline{\theta}_{y}}{2}\right)-\sin \left({ }^{t} \theta_{y}\right) \sin \left(\frac{\underline{\theta}_{y}}{2}\right)\right] \\
& \underline{\hat{v}}=\underline{v}-2{ }^{0} z \sin \left(\frac{\underline{\theta}_{x}}{2}\right)\left[\cos \left({ }^{t} \theta_{x}\right) \cos \left(\frac{\underline{\theta}_{x}}{2}\right)-\sin \left({ }^{t} \theta_{x}\right) \sin \left(\frac{\underline{\theta}_{x}}{2}\right)\right] \\
& \underline{\hat{w}}=\underline{w}
\end{aligned}
$$

with 


$$
\begin{aligned}
& \underline{\theta}_{x}={ }^{t+\Delta t} \theta_{x}-{ }^{t} \theta_{x} \\
& \underline{\theta}_{y}={ }^{t+\Delta t} \theta_{y}-{ }^{t} \theta_{y}
\end{aligned}
$$

where $\underline{\theta}_{x}$ and $\underline{\theta}_{y}$ represent the incremental rotations from time $t$ to time $t+\Delta t$ of a material point located on the mid-plane of the plate (see Fig. 3(a)).

Similar to the approximation in nonlinear FEA [1], the incremental rotations $\underline{\theta}_{x}$ and $\underline{\theta}_{y}$ can be assumed very small as

$$
\sin \left(\frac{\underline{\theta}_{y}}{2}\right) \approx \frac{\underline{\theta}_{y}}{2}, \quad \cos \left(\frac{\underline{\theta}_{y}}{2}\right) \approx 1, \quad \sin ^{2}\left(\frac{\underline{\theta}_{y}}{2}\right) \approx 0
$$

Therefore, the incremental displacements at any point $P$ located at $\left({ }^{0} x,{ }^{0} y,{ }^{0} z\right)$ provided in Eqs. (24a-c) can be simplified as

$$
\begin{aligned}
& \underline{\hat{u}}=\underline{u}+{ }^{0} z \underline{\theta}_{y} \cos \left({ }^{t} \theta_{y}\right) \\
& \underline{\hat{v}}=\underline{v}-{ }^{0} z \underline{\theta}_{x} \cos \left({ }^{t} \theta_{x}\right) \\
& \underline{\hat{w}}=\underline{w}
\end{aligned}
$$

\subsection{Strain field}

By using the displacements given in Eq. (20), the Green-Lagrange strain at time $t$ given in Eqs. (4c-g) for a material point located at $\left({ }^{0} x,{ }^{0} y,{ }^{0} z\right)$ can be represented as [64]

$$
\begin{aligned}
& { }_{0}^{t} \hat{\varepsilon}_{x x}={ }_{0}^{t} u_{, x}+{ }^{0} z \frac{\partial\left(\sin { }^{t} \theta_{y}\right)}{\partial^{0} x}+\frac{1}{2}\left[{ }_{0}^{t} u_{, x}{ }^{2}+{ }_{0}^{t} v_{, x}{ }^{2}+{ }_{0}^{t} w_{, x}{ }^{2}\right] \\
& +\frac{1}{2}\left[{ }^{0} z \frac{\partial\left(\sin ^{t} \theta_{y}\right)}{\partial^{0} x}\left({ }^{0} z \frac{\partial\left(\sin ^{t} \theta_{y}\right)}{\partial^{0} x}+2{ }_{0}^{t} u_{, x}\right)+{ }^{0} z \frac{\partial\left(\sin ^{t} \theta_{x}\right)}{\partial^{0} x}\left({ }^{0} z \frac{\partial\left(\sin ^{t} \theta_{x}\right)}{\partial^{0} x}-2{ }_{0}^{t} v_{, x}\right)\right] \\
& { }_{0}^{t} \hat{\varepsilon}_{y y}={ }_{0}^{t} v_{, y}-{ }^{0} z \frac{\partial\left(\sin ^{t} \theta_{x}\right)}{\partial^{0} y}+\frac{1}{2}\left[{ }_{0}^{t} u_{, y}{ }^{2}+{ }_{0}^{t} v_{, y}{ }^{2}+{ }_{0}^{t} w_{, y}{ }^{2}\right] \\
& +\frac{1}{2}\left[{ }_{0}^{0} z \frac{\partial\left(\sin ^{t} \theta_{y}\right)}{\partial^{0} y}\left(2{ }_{0}^{t} u_{, y}+{ }^{0} z \frac{\partial\left(\sin ^{t} \theta_{y}\right)}{\partial^{0} y}\right)+{ }^{0} z \frac{\partial\left(\sin ^{t} \theta_{x}\right)}{\partial^{0} y}\left({ }^{0} z \frac{\partial\left(\sin ^{t} \theta_{x}\right)}{\partial^{0} y}-2{ }_{0}^{t} v_{, y}\right)\right] \\
& { }_{0}^{t} \hat{\varepsilon}_{x y}=\frac{1}{2}\left[{ }_{0}^{t} u_{, y}+{ }^{0} z \frac{\partial\left(\sin ^{t} \theta_{y}\right)}{\partial^{0} y}+{ }_{0}^{t} v_{, x}-{ }^{0} z \frac{\partial\left(\sin ^{t} \theta_{x}\right)}{\partial^{0} x}+{ }_{0}^{t} u_{, x}{ }_{0}^{t} u_{, y}+{ }_{0}^{t} v_{, x}{ }_{0}^{t} v_{, y}+{ }_{0}^{t} w_{, x 0}{ }^{t} w_{, y}\right] \\
& +\frac{1}{2}\left[\begin{array}{c}
{ }^{0} z \frac{\partial\left(\sin ^{t} \theta_{y}\right)}{\partial^{0} x}\left({ }_{0}^{t} u_{, y}+{ }^{0} z \frac{\partial\left(\sin ^{t} \theta_{y}\right)}{\partial^{0} y}\right)+\left({ }_{0}^{t} u_{, x}+{ }^{0} z \frac{\partial\left(\sin ^{t} \theta_{y}\right)}{\partial^{0} x}\right) 0 \frac{\partial\left(\sin ^{t} \theta_{y}\right)}{\partial^{0} y} \\
-{ }^{0} z \frac{\partial\left(\sin ^{t} \theta_{x}\right)}{\partial^{0} x}\left({ }_{0}^{t} v_{, y}-{ }^{0} z \frac{\partial\left(\sin ^{t} \theta_{x}\right)}{\partial^{0} y}\right)-\left({ }_{0}^{t} v_{, x}-{ }^{0} z \frac{\partial\left(\sin ^{t} \theta_{x}\right)}{\partial^{0} x}\right) 0 \frac{\partial\left(\sin ^{t} \theta_{x}\right)}{\partial^{0} y}
\end{array}\right]
\end{aligned}
$$




$$
\begin{aligned}
{ }_{0}^{t} \hat{\varepsilon}_{x z}= & \frac{1}{2}\left[{ }_{0}^{t} w_{, x}+\sin \left({ }^{t} \theta_{y}\right)+{ }_{0}^{t} u_{, x} \sin \left({ }^{t} \theta_{y}\right)-{ }_{0}^{t} v_{, x} \sin \left({ }^{t} \theta_{x}\right)\right] \\
& +\frac{1}{2}\left[{ }^{0} z_{0}^{t} \theta_{y, x} \sin \left({ }^{t} \theta_{y}\right)+{ }^{0} z_{0}^{t} \theta_{x, x} \sin \left({ }^{t} \theta_{x}\right)\right] \\
{ }_{0}^{t} \hat{\varepsilon}_{y z}= & \frac{1}{2}\left[{ }_{0}^{t} w_{, y}-\sin \left({ }^{t} \theta_{x}\right)+{ }_{0}^{t} u_{, y} \sin \left({ }^{t} \theta_{y}\right)-{ }_{0}^{t} v_{, y} \sin \left({ }^{t} \theta_{x}\right)\right] \\
& +\frac{1}{2}\left[{ }^{0} z_{0}^{t} \theta_{y, y} \sin \left({ }^{t} \theta_{y}\right)+{ }^{0} z_{0}^{t} \theta_{x, y} \sin \left({ }^{t} \theta_{x}\right)\right] \\
{ }_{0}^{t} \hat{\varepsilon}_{z z} \approx 0 &
\end{aligned}
$$

with

$$
\begin{aligned}
& { }_{0}^{t} u_{, x}=\frac{\partial^{t} u}{\partial^{0} x} ;{ }_{0}^{t} u_{, y}=\frac{\partial^{t} u}{\partial^{0} y} \\
& { }_{0}^{t} v_{, x}=\frac{\partial^{t} v}{\partial^{0} x} ;{ }_{0}^{t} v_{, y}=\frac{\partial^{t} v}{\partial^{0} y} \\
& { }_{0}^{t} w_{, x}=\frac{\partial^{t} w}{\partial^{0} x} ;{ }_{0}^{t} w_{, y}=\frac{\partial^{t} w}{\partial^{0} y} \\
& { }_{0}^{t} \theta_{x, x}=\frac{\partial^{t} \theta_{x}}{\partial^{0} x} ;{ }_{0}^{t} \theta_{x, y}=\frac{\partial^{t} \theta_{x}}{\partial^{0} y} \\
& { }_{0}^{t} \theta_{y, x}=\frac{\partial^{t} \theta_{y}}{\partial^{0} x} ;{ }_{0}^{t} \theta_{y, y}=\frac{\partial^{t} \theta_{y}}{\partial^{0} y}
\end{aligned}
$$

Note that, the relation given in Eq. (27f) is applicable for small strain problems. In the small strain conditions, the thickness of the plate is assumed as unchanged. Therefore, the strain in the thickness direction of the plate is ignored.

By assuming the plate is moderately thin, the following terms in Eq. (27) can be neglected as [64]

$$
\begin{aligned}
& \frac{1}{2}\left[{ }^{0} z \frac{\partial\left(\sin ^{t} \theta_{y}\right)}{\partial^{0} x}\left({ }^{0} z \frac{\partial\left(\sin ^{t} \theta_{y}\right)}{\partial^{0} x}+2{ }_{0}^{t} u_{, x}\right)+{ }^{0} z \frac{\partial\left(\sin ^{t} \theta_{x}\right)}{\partial^{0} x}\left({ }^{0} z \frac{\partial\left(\sin ^{t} \theta_{x}\right)}{\partial^{0} x}-2{ }_{0}^{t} v_{, x}\right)\right] \rightarrow 0 \\
& \frac{1}{2}\left[{ }^{0} z \frac{\partial\left(\sin ^{t} \theta_{y}\right)}{\partial^{0} y}\left(2{ }_{0}^{t} u_{, y}+{ }^{0} z \frac{\partial\left(\sin ^{t} \theta_{y}\right)}{\partial^{0} y}\right)+{ }^{0} z \frac{\partial\left(\sin ^{t} \theta_{x}\right)}{\partial^{0} y}\left({ }^{0} z \frac{\partial\left(\sin ^{t} \theta_{x}\right)}{\partial^{0} y}-2{ }_{0}^{t} v_{, y}\right)\right] \rightarrow 0 \\
& \frac{1}{2}\left[\begin{array}{c}
{ }^{0} z \frac{\partial\left(\sin ^{t} \theta_{y}\right)}{\partial^{0} x}\left({ }_{0}^{t} u_{, y}+{ }^{0} z \frac{\partial\left(\sin ^{t} \theta_{y}\right)}{\partial^{0} y}\right)+\left({ }_{0}^{t} u_{, x}+{ }^{0} z \frac{\partial\left(\sin ^{t} \theta_{y}\right)}{\partial^{0} x}\right){ }^{0} z \frac{\partial\left(\sin ^{t} \theta_{y}\right)}{\partial^{0} y} \\
-{ }^{0} z \frac{\partial\left(\sin ^{t} \theta_{x}\right)}{\partial^{0} x}\left({ }_{0}^{t} v_{, y}-{ }^{0} z \frac{\partial\left(\sin ^{t} \theta_{x}\right)}{\partial^{0} y}\right)-\left({ }_{0}^{t} v_{, x}-{ }^{0} z \frac{\partial\left(\sin ^{t} \theta_{x}\right)}{\partial^{0} x}\right){ }^{0} z \frac{\partial\left(\sin ^{t} \theta_{x}\right)}{\partial^{0} y}
\end{array}\right] \rightarrow 0
\end{aligned}
$$




$$
\begin{aligned}
& \frac{1}{2}\left[{ }^{0} z_{0}^{t} \theta_{y, x} \sin \left({ }^{t} \theta_{y}\right)+{ }^{0} z_{0}^{t} \theta_{x, x} \sin \left({ }^{t} \theta_{x}\right)\right] \rightarrow 0 \\
& \frac{1}{2}\left[{ }^{0} z_{0}^{t} \theta_{y, y} \sin \left({ }^{t} \theta_{y}\right)+{ }^{0} z_{0}^{t} \theta_{x, y} \sin \left({ }^{t} \theta_{x}\right)\right] \rightarrow 0
\end{aligned}
$$

Therefore, the Green-Lagrange strains in Eq. (27) can be simplified as [64]

$$
\begin{aligned}
& { }_{0}^{t} \hat{\varepsilon}_{x x}={ }_{0}^{t} u_{, x}+{ }^{0} z \frac{\partial\left(\sin ^{t} \theta_{y}\right)}{\partial^{0} x}+\frac{1}{2}\left({ }_{0}^{t} u_{, x}{ }^{2}+{ }_{0}^{t} v_{, x}{ }^{2}+{ }_{0}^{t} w_{, x}{ }^{2}\right) \\
& { }_{0}^{t} \hat{\varepsilon}_{y y}={ }_{0}^{t} v_{, y}-{ }^{0} z \frac{\partial\left(\sin ^{t} \theta_{x}\right)}{\partial^{0} y}+\frac{1}{2}\left({ }_{0}^{t} u_{, y}{ }^{2}+{ }_{0}^{t} v_{, y}{ }^{2}+{ }_{0}^{t} w_{, y}{ }^{2}\right) \\
& { }_{0}^{t} \hat{\varepsilon}_{x y}=\frac{1}{2}\left[{ }_{0}^{t} u_{, y}+{ }_{0}^{t} v_{, x}+{ }^{0} z\left(\frac{\partial\left(\sin { }^{t} \theta_{y}\right)}{\partial^{0} y}-\frac{\partial\left(\sin { }^{t} \theta_{x}\right)}{\partial^{0} x}\right)+{ }_{0}^{t} u_{, x}{ }_{0}^{t} u_{, y}+{ }_{0}^{t} v_{, x}{ }_{0}^{t} v_{, y}+{ }_{0}^{t} w_{, x}{ }_{0}^{t} w_{, y}\right] \\
& { }_{0}^{t} \hat{\varepsilon}_{x z}=\frac{1}{2}\left[{ }_{0}^{t} w_{, x}+\sin \left({ }^{t} \theta_{y}\right)+{ }_{0}^{t} u_{, x} \sin \left({ }^{t} \theta_{y}\right)-{ }_{0}^{t} v_{, x} \sin \left({ }^{t} \theta_{x}\right)\right] \\
& { }_{0}^{t} \hat{\varepsilon}_{y z}=\frac{1}{2}\left[{ }_{0}^{t} w_{, y}-\sin \left({ }^{t} \theta_{x}\right)+{ }_{0}^{t} u_{, y} \sin \left({ }^{t} \theta_{y}\right)-{ }_{0}^{t} v_{, y} \sin \left({ }^{t} \theta_{x}\right)\right] \\
& { }_{0}^{t} \hat{\varepsilon}_{z z} \approx 0
\end{aligned}
$$

On the other hand, by substituting the displacement fields given in Eq. (20) and Eq. (26) into incremental Green-Lagrange strain, ${ }_{0} \hat{\underline{e}}_{i j}$ in Eq. (9) and by neglecting higher-order terms, the linear components of the incremental Green-Lagrange strain, ${ }_{0} \underline{\hat{e}}_{i j}$, can be represented as [64]

$$
\begin{aligned}
& { }_{0} \underline{\hat{e}}_{x x}={ }_{0} \underline{u}_{, x}+{ }^{0} z \frac{\partial\left(\underline{\theta}_{y} \cos { }^{t} \theta_{y}\right)}{\partial^{0} x}+{ }_{0} \underline{u}_{, x}{ }_{0}^{t} u_{, x}+{ }_{0} \underline{v}_{, x}{ }_{0}^{t} v_{, x}+{ }_{0} \underline{w}_{, x}{ }_{0}^{t} w_{, x} \\
& { }_{0} \underline{\hat{e}}_{y y}={ }_{0} \underline{v}, y={ }^{0} z \frac{\partial\left(\underline{\theta}_{x} \cos { }^{t} \theta_{x}\right)}{\partial^{0} y}+{ }_{0} \underline{u}, y{ }_{0}^{t} u_{, y}+{ }_{0} \underline{v}, y{ }_{0}^{t} v_{, y}+{ }_{0} \underline{w}_{, y}{ }_{0}^{t} w_{, y} \\
& { }_{0} \underline{\hat{e}}_{x y}=\frac{1}{2}\left[\begin{array}{l}
{ }_{0} \underline{u}, y_{, y}+{ }_{0} \underline{v}_{, x}+{ }^{0} z \frac{\partial\left(\underline{\theta}_{y} \cos { }^{t} \theta_{y}\right)}{\partial^{0} y}-{ }^{0} z \frac{\partial\left(\underline{\theta}_{x} \cos { }^{t} \theta_{x}\right)}{\partial^{0} x} \\
+{ }_{0} \underline{u}_{, x}{ }_{0}^{t} u_{, y}+{ }_{0}^{t} u_{, x} \underline{u}_{, y}+{ }_{0} \underline{v}_{, x}{ }_{0}^{t} v_{, y}+{ }_{0}^{t} v_{, x} 0 \underline{v}, y_{, y}+{ }_{0} \underline{w}_{, x}{ }_{0}^{t} w_{, y}+{ }_{0}^{t} w_{, x} 0 \underline{w}_{, y}
\end{array}\right] \\
& { }_{0} \underline{\hat{e}}_{x z}=\frac{1}{2}\left[{ }_{0} \underline{w}_{, x}+\underline{\theta}_{y} \cos \left({ }^{t} \theta_{y}\right)\right] \\
& { }_{0} \underline{\hat{e}}_{y z}=\frac{1}{2}\left[{ }_{0} \underline{w}_{, y}-\underline{\theta}_{x} \cos \left({ }^{t} \theta_{x}\right)\right]
\end{aligned}
$$

with

$$
{ }_{0} \underline{u}_{, x}=\frac{\partial \underline{u}}{\partial^{0} x} ;{ }_{0} \underline{u}_{, y}=\frac{\partial \underline{u}}{\partial^{0} y}
$$




$$
\begin{aligned}
& { }_{0} \underline{v}, x_{, x}=\frac{\partial \underline{v}}{\partial^{0} x} ;{ }_{0} \underline{v}, y_{y}=\frac{\partial \underline{v}}{\partial^{0} y} \\
& { }_{0} \underline{w}_{, x}=\frac{\partial \underline{w}}{\partial^{0} x} ;{ }_{0} \underline{w}_{, y}=\frac{\partial \underline{w}}{\partial^{0} y}
\end{aligned}
$$

\subsection{Second Piola-Kirchhoff stress}

By substituting the Green-Lagrange strain at time $t$ given in Eq. (29) into Eq. (11), the second Piola-Kirchhoff stress components for a plate with large displacements, large rotations, but small elastic strains can be calculated as [64]

$$
\begin{aligned}
& { }_{0}^{t} \hat{S}_{x x}=\frac{E}{1-v^{2}}\left(\begin{array}{l}
{ }_{0}^{t} u_{, x}+v_{0}^{t} v_{, y}+{ }^{0} z\left(\frac{\partial\left(\sin ^{t} \theta_{y}\right)}{\partial{ }^{0} x}-v \frac{\partial\left(\sin ^{t} \theta_{x}\right)}{\partial^{0} y}\right) \\
+\frac{1}{2}\left[\left({ }_{0}^{t} u_{, x}{ }^{2}+{ }_{0}^{t} v_{, x}{ }^{2}+{ }_{0}^{t} w_{, x}{ }^{2}\right)+v\left({ }_{0}^{t} u_{, y}{ }^{2}+{ }_{0}^{t} v_{, y}{ }^{2}+{ }_{0}^{t} w_{, y}{ }^{2}\right)\right]
\end{array}\right) \\
& { }_{0}^{t} \hat{S}_{y y}=\frac{E}{1-v^{2}}\left(\begin{array}{l}
{ }_{0}^{t} v_{, y}+v_{0}^{t} u_{, x}-{ }^{0} z\left(\frac{\partial\left(\sin ^{t} \theta_{x}\right)}{\partial^{0} y}-v \frac{\partial\left(\sin ^{t} \theta_{y}\right)}{\partial^{0} y}\right) \\
+\frac{1}{2}\left[\left({ }_{0}^{t} u_{, y}{ }^{2}+{ }_{0}^{t} v_{, y}{ }^{2}+{ }_{0}^{t} w_{, y}{ }^{2}\right)+v\left({ }_{0}^{t} u_{, x}{ }^{2}+{ }_{0}^{t} v_{, x}{ }^{2}+{ }_{0}^{t} w_{, x}{ }^{2}\right)\right]
\end{array}\right) \\
& { }_{0}^{t} \hat{S}_{x y}=\frac{E}{2(1+v)}\left[{ }_{0}^{t} u_{, y}+{ }_{0}^{t} v_{, x}+{ }^{0} z\left(\frac{\partial\left(\sin { }^{t} \theta_{y}\right)}{\partial^{0} y}-\frac{\partial\left(\sin { }^{t} \theta_{x}\right)}{\partial^{0} x}\right)+{ }_{0}^{t} u_{, x}{ }_{0}^{t} u_{, y}+{ }_{0}^{t} v_{, x}{ }_{0}^{t} v_{, y}+{ }_{0}^{t} w_{, x}{ }_{0}^{t} w_{, y}\right] \\
& { }_{0}^{t} \hat{S}_{x z}=\frac{k_{s} E}{2(1+v)}\left[{ }_{0}^{t} w_{, x}+\sin \left({ }^{t} \theta_{y}\right)\right] \\
& { }_{0}^{t} \hat{S}_{y z}=\frac{k_{s} E}{2(1+v)}\left[{ }_{0}^{t} w_{, y}-\sin \left({ }^{t} \theta_{x}\right)\right] \\
& { }_{0}^{t} \hat{S}_{z z} \approx 0
\end{aligned}
$$

\subsection{The strain energy density}

By substituting the second Piola-Kirchhoff stress components given in Eq. (31) and the incremental strains given in Eq. (30) into Eq. (18), the SED of a plate can be represented as [64] 


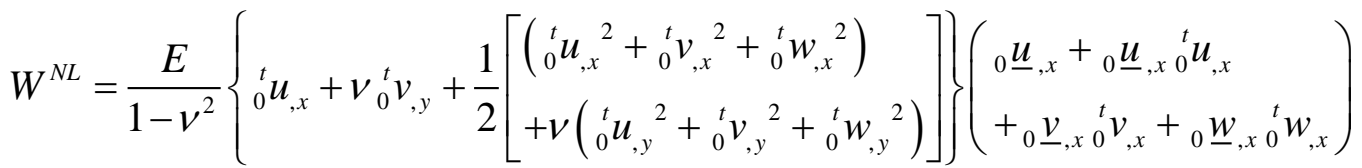

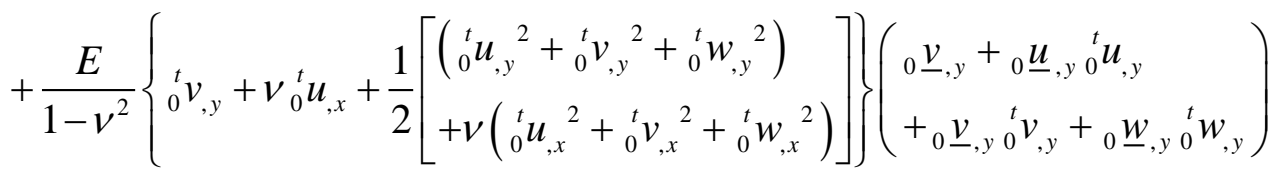

$$
\begin{aligned}
& +\frac{E}{2(1+v)}\left(\begin{array}{c}
{ }_{0}^{t} u_{, y}+{ }_{0}^{t} v_{, x}+{ }_{0}^{t} u_{, x}{ }_{0}^{t} u_{, y} \\
+{ }_{0}^{t} v_{, x}{ }_{0}^{t} v_{, y}+{ }_{0}^{t} w_{, x}{ }^{t} w_{, y}
\end{array}\right)\left(\begin{array}{l}
0 \underline{u}_{, y}+{ }_{0} \underline{v}_{, x}+{ }_{0} \underline{u}_{, x}{ }_{0}^{t} u_{, y}+{ }_{0}^{t} u_{, x 0} \underline{u}, y \\
+{ }_{0} \underline{v}_{, x}{ }_{0}^{t} v_{, y}+{ }_{0}^{t} v_{, x 0} \underline{v}_{, y}+{ }_{0} \underline{w}_{, x}{ }_{0}^{t} w_{, y}+{ }_{0}^{t} w_{, x 0} \underline{w}_{, y}
\end{array}\right) \\
& +\frac{k_{s} E}{2(1+v)}\left[\begin{array}{l}
\left({ }_{0}^{t} w_{, x}+\sin \left({ }^{t} \theta_{y}\right)\right)\left({ }_{0} \underline{w}_{, x}+\underline{\theta}_{y} \cos \left({ }^{t} \theta_{y}\right)\right) \\
+\left({ }_{0}^{t} w_{, y}-\sin \left({ }^{t} \theta_{x}\right)\right)\left({ }_{0} \underline{w}_{, y}-\underline{\theta}_{x} \cos \left({ }^{t} \theta_{x}\right)\right)
\end{array}\right] \\
& +\frac{E}{1-v^{2}}\left({ }^{0} z\right)^{2}\left(\begin{array}{l}
\left(\frac{\partial\left(\sin ^{t} \theta_{y}\right)}{\partial^{0} x}-v \frac{\partial\left(\sin ^{t} \theta_{x}\right)}{\partial^{0} y}\right) \frac{\partial\left(\underline{\theta}_{y} \cos \left({ }^{t} \theta_{y}\right)\right)}{\partial^{0} x} \\
+\left(\frac{\partial\left(\sin ^{t} \theta_{x}\right)}{\partial^{0} y}-v \frac{\partial\left(\sin ^{t} \theta_{y}\right)}{\partial^{0} x}\right) \frac{\partial\left(\underline{\theta}_{x} \cos \left({ }^{t} \theta_{x}\right)\right)}{\partial^{0} y}
\end{array}\right) \\
& +\frac{E}{2(1+v)}\left({ }^{0} z\right)^{2}\left(\frac{\partial\left(\sin ^{t} \theta_{y}\right)}{\partial^{0} y}-\frac{\partial\left(\sin ^{t} \theta_{x}\right)}{\partial^{0} x}\right)\left(\frac{\partial\left(\underline{\theta}_{y} \cos \left({ }^{t} \theta_{y}\right)\right)}{\partial^{0} y}-\frac{\partial\left(\underline{\theta}_{x} \cos \left({ }^{t} \theta_{x}\right)\right)}{\partial^{0} x}\right)
\end{aligned}
$$

The strain energy per unit area, $\bar{W}^{N L}$ can be obtained by integrating the SED given in Eq. (32) through the thickness of the plate as $[1,3]$ 


$$
\begin{aligned}
& \bar{W}^{N L}=\int_{-h / 2}^{h / 2} W^{N L} d^{0} z
\end{aligned}
$$

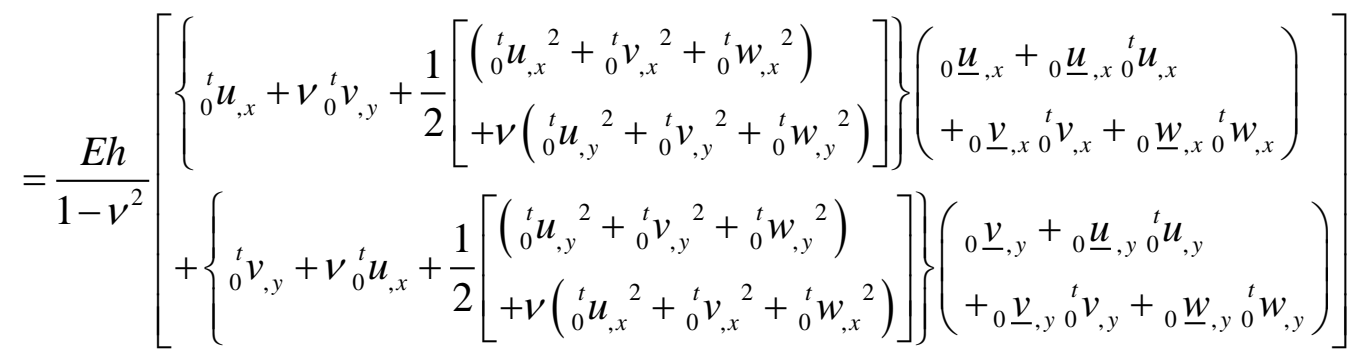

$$
\begin{aligned}
& +\frac{E h}{2(1+v)}\left(\begin{array}{l}
{ }_{0}^{t} u_{, y}+{ }_{0}^{t} v_{, x}+{ }_{0}^{t} u_{, x}{ }_{0}^{t} u_{, y} \\
+{ }_{0}^{t} v_{, x 0}{ }_{t}^{t} v_{, y}+{ }_{0}^{t} w_{, x 0}{ }^{t} w_{, y}
\end{array}\right)\left(\begin{array}{l}
0 \underline{u}_{, y}+{ }_{0} \underline{v}_{, x}+{ }_{0} \underline{u}_{, x}{ }_{0}^{t} u_{, y}+{ }_{0}^{t} u_{, x 0} \underline{u}, y \\
+{ }_{0} \underline{v}_{, x}{ }^{t} v_{, y}+{ }_{0}^{t} v_{, x 0} \underline{v}_{, y}+{ }_{0} \underline{w}_{, x 0}{ }^{t} w_{, y}+{ }_{0}^{t} w_{, x 0} \underline{w}_{, y}
\end{array}\right) \\
& +\frac{k_{s} E h}{2(1+v)}\left[\begin{array}{l}
\left({ }_{0}^{t} w_{, x}+\sin \left({ }^{t} \theta_{y}\right)\right)\left({ }_{0} \underline{w}_{, x}+\underline{\theta}_{y} \cos \left({ }^{t} \theta_{y}\right)\right) \\
+\left({ }_{0}^{t} w_{, y}-\sin \left({ }^{t} \theta_{x}\right)\right)\left({ }_{0} \underline{w}, y_{-}-\underline{\theta}_{x} \cos \left({ }^{t} \theta_{x}\right)\right)
\end{array}\right] \\
& +\frac{E h^{3}}{12\left(1-v^{2}\right)}\left(\begin{array}{l}
\left(\frac{\partial\left(\sin ^{t} \theta_{y}\right)}{\partial^{0} x}-v \frac{\partial\left(\sin ^{t} \theta_{x}\right)}{\partial^{0} y}\right) \frac{\partial\left(\underline{\theta}_{y} \cos \left({ }^{t} \theta_{y}\right)\right)}{\partial^{0} x} \\
+\left(\frac{\partial\left(\sin ^{t} \theta_{x}\right)}{\partial^{0} y}-v \frac{\partial\left(\sin ^{t} \theta_{y}\right)}{\partial^{0} x}\right) \frac{\partial\left(\underline{\theta}_{x} \cos \left({ }^{t} \theta_{x}\right)\right)}{\partial^{0} y}
\end{array}\right) \\
& +\frac{E h^{3}}{24(1+v)}\left(\frac{\partial\left(\sin ^{t} \theta_{y}\right)}{\partial^{0} y}-\frac{\partial\left(\sin ^{t} \theta_{x}\right)}{\partial^{0} x}\right)\left(\frac{\partial\left(\underline{\theta}_{y} \cos \left({ }^{t} \theta_{y}\right)\right)}{\partial^{0} y}-\frac{\partial\left(\underline{\theta}_{x} \cos \left({ }^{t} \theta_{x}\right)\right)}{\partial^{0} x}\right)
\end{aligned}
$$

The strain energy per unit area given in Eq. (33) can be rewritten as 


$$
\begin{aligned}
& \bar{W}^{N L}=\frac{E h}{1-v^{2}}\left({ }_{0}^{t} \vartheta\right)\left({ }_{0} \underline{\vartheta}\right) \\
& +\frac{E h}{2(1+v)}\left[\begin{array}{l}
\left(\begin{array}{l}
{ }_{0}^{t} u_{, y}+{ }_{0}^{t} v_{, x}+{ }_{0}^{t} u_{, x}{ }_{0}^{t} u_{, y} \\
+{ }_{0}^{t} v_{, x}{ }_{0}^{t} v_{, y}+{ }_{0}^{t} w_{, x}{ }_{0}^{t} w_{, y}
\end{array}\right)\left(\begin{array}{l}
0 \underline{u}_{, y}+{ }_{0} \underline{v}_{, x}+{ }_{0} \underline{u}_{, x}{ }_{0}^{t} u_{, y}+{ }_{0}^{t} u_{, x 0} \underline{u}_{, y} \\
+{ }_{0} \underline{v}_{, x}{ }_{0}^{t} v_{, y}+{ }_{0}^{t} v_{, x 0} \underline{v}_{, y}+{ }_{0} \underline{w}_{, x}{ }_{0}^{t} w_{, y}+{ }_{0}^{t} w_{, x} \underline{w}_{, y}
\end{array}\right) \\
-2\left[\begin{array}{l}
\left.{ }_{0}^{t} \varepsilon_{y y}\left(\begin{array}{l}
0 \underline{u}_{, x}+{ }_{0} \underline{u}_{, x}{ }_{0}^{t} u_{, x} \\
+{ }_{0} \underline{v}_{, x}{ }_{0}^{t} v_{, x}+{ }_{0} \underline{w}_{, x}{ }_{0}^{t} w_{, x}
\end{array}\right)+{ }_{0}^{t} \varepsilon_{x x}\left(\begin{array}{l}
0 \underline{v}_{, y}+{ }_{0} \underline{u}, y{ }_{0} u_{, y} \\
+_{0} \underline{v}_{, y}{ }_{0}^{t} v_{, y}+{ }_{0} \underline{w}_{, y}{ }_{0}^{t} w_{, y}
\end{array}\right)\right]
\end{array}\right]
\end{array}\right. \\
& +\frac{k_{s} E h}{2(1+v)}\left[\begin{array}{l}
\left({ }_{0}^{t} w_{, x}+\sin \left({ }^{t} \theta_{y}\right)\right)\left({ }_{0} \underline{w}, x_{, x}+\underline{\theta}_{y} \cos \left({ }^{t} \theta_{y}\right)\right) \\
+\left({ }_{0}^{t} w_{, y}-\sin \left({ }^{t} \theta_{x}\right)\right)\left({ }_{0} \underline{w}, y_{-}-\underline{\theta}_{x} \cos \left({ }^{t} \theta_{x}\right)\right)
\end{array}\right] \\
& +\frac{E h^{3}}{12\left(1-v^{2}\right)}\left[\begin{array}{l}
\left(\frac{\partial\left(\sin ^{t} \theta_{y}\right)}{\partial^{0} x}-\frac{\partial\left(\sin ^{t} \theta_{x}\right)}{\partial^{0} y}\right)\left(\frac{\partial\left(\underline{\theta}_{y} \cos \left({ }^{t} \theta_{y}\right)\right)}{\partial^{0} x}-\frac{\partial\left(\underline{\theta}_{x} \cos \left({ }^{t} \theta_{x}\right)\right)}{\partial^{0} y}\right) \\
+\frac{1-v}{2}\left[\begin{array}{l}
\left(\frac{\partial\left(\sin ^{t} \theta_{y}\right)}{\partial^{0} y}-\frac{\partial\left(\sin ^{t} \theta_{x}\right)}{\partial^{0} x}\right)\left(\frac{\partial\left(\underline{\theta}_{y} \cos \left({ }^{t} \theta_{y}\right)\right)}{\partial^{0} y}-\frac{\partial\left(\underline{\theta}_{x} \cos \left({ }^{t} \theta_{x}\right)\right)}{\partial^{0} x}\right) \\
+2 \frac{\partial\left(\sin ^{t} \theta_{x}\right)}{\partial^{0} y} \frac{\partial\left(\underline{\theta}_{y} \cos \left({ }^{t} \theta_{y}\right)\right)}{\partial^{0} x}+2 \frac{\partial\left(\sin ^{t} \theta_{y}\right)}{\partial^{0} x} \frac{\partial\left(\underline{\theta}_{x} \cos \left({ }^{t} \theta_{x}\right)\right)}{\partial^{0} y}
\end{array}\right]
\end{array}\right]
\end{aligned}
$$

with

$$
\begin{aligned}
& { }_{0}^{t} \varepsilon_{x x}={ }_{0}^{t} u_{, x}+\frac{1}{2}\left({ }_{0}^{t} u_{, x}{ }^{2}+{ }_{0}^{t} v_{, x}{ }^{2}+{ }_{0}^{t} w_{, x}{ }^{2}\right) \\
& { }_{0}^{t} \varepsilon_{y y}={ }_{0}^{t} v_{, y}+\frac{1}{2}\left({ }_{0}^{t} u_{, y}{ }^{2}+{ }_{0}^{t} v_{, y}{ }^{2}+{ }_{0}^{t} w_{, y}{ }^{2}\right) \\
& { }_{0}^{t} \vartheta={ }_{0}^{t} \varepsilon_{x x}+{ }_{0}^{t} \varepsilon_{y y}={ }_{0}^{t} u_{, x}+{ }_{0}^{t} v_{, y}+\frac{1}{2}\left({ }_{0}^{t} u_{, x}{ }^{2}+{ }_{0}^{t} v_{, x}{ }^{2}+{ }_{0}^{t} w_{, x}{ }^{2}\right)+\frac{1}{2}\left({ }_{0}^{t} u_{, y}{ }^{2}+{ }_{0}^{t} v_{, y}{ }^{2}+{ }_{0}^{t} w_{, y}{ }^{2}\right) \\
& { }_{0} \underline{\vartheta}={ }_{0} \underline{u}_{, x}+{ }_{0} \underline{v}_{, y}+{ }_{0} \underline{u}, x 0 \\
& { }_{0}^{t} u_{, x}+{ }_{0} \underline{u}, y{ }_{0}^{t} u_{, y}+{ }_{0} \underline{v}_{, x}{ }_{0}^{t} v_{, x}+{ }_{0} \underline{v}_{, y}{ }_{0}^{t} v_{, y}+{ }_{0} \underline{w}_{, x}{ }_{0}^{t} w_{, x}+{ }_{0} \underline{w}_{, y}{ }_{0}^{t} w_{, y}
\end{aligned}
$$

The strain energy per unit area given in Eq. (34a) can also be decomposed as [27]

$\bar{W}^{N L}=\bar{W}_{i p}^{N L}+\bar{W}_{s h}^{N L}+\bar{W}_{b}^{N L}$

with

$$
\begin{aligned}
& \bar{W}_{i p}^{N L}=\frac{E h}{1-v^{2}}\left({ }_{0}^{t} \vartheta\right)\left({ }_{0} \underline{\vartheta}\right)
\end{aligned}
$$

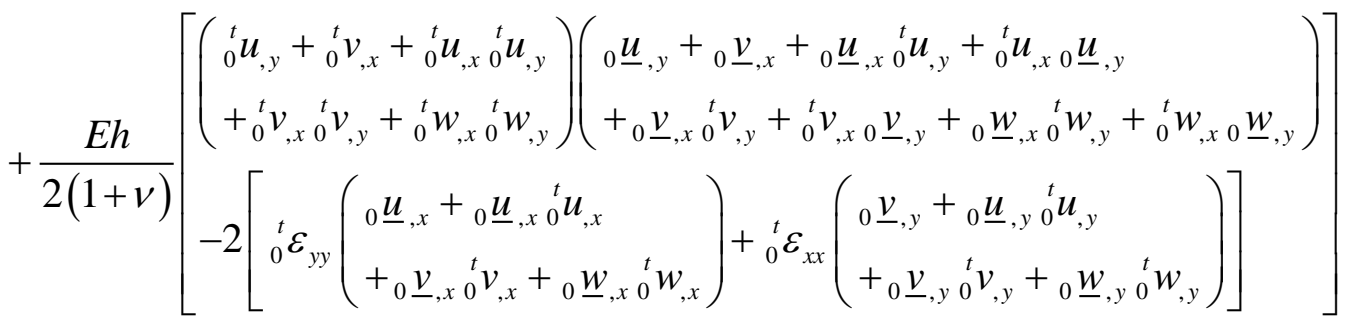




$$
\begin{aligned}
& \bar{W}_{s h}^{N L}=\frac{k_{s} E h}{2(1+v)}\left[\begin{array}{l}
\left({ }_{0}^{t} w_{, x}+\sin \left({ }^{t} \theta_{y}\right)\right)\left({ }_{0} \underline{w}_{, x}+\underline{\theta}_{y} \cos \left({ }^{t} \theta_{y}\right)\right) \\
+\left({ }_{0}^{t} w_{, y}-\sin \left({ }^{t} \theta_{x}\right)\right)\left({ }_{0} \underline{w}_{, y}-\underline{\theta}_{x} \cos \left({ }^{t} \theta_{x}\right)\right)
\end{array}\right] \\
& \bar{W}_{b}^{N L}=\frac{E h^{3}}{12\left(1-v^{2}\right)}\left[\begin{array}{l}
\left(\frac{\partial\left(\sin ^{t} \theta_{y}\right)}{\partial^{0} x}-\frac{\partial\left(\sin ^{t} \theta_{x}\right)}{\partial^{0} y}\right)\left(\frac{\partial\left(\underline{\theta}_{y} \cos \left({ }^{t} \theta_{y}\right)\right)}{\partial^{0} x}-\frac{\partial\left(\underline{\theta}_{x} \cos \left({ }^{t} \theta_{x}\right)\right)}{\partial^{0} y}\right) \\
+\frac{1-v}{2}\left[\begin{array}{l}
\left(\frac{\partial\left(\sin ^{t} \theta_{y}\right)}{\partial^{0} y}-\frac{\partial\left(\sin ^{t} \theta_{x}\right)}{\partial^{0} x}\right)\left(\frac{\partial\left(\underline{\theta}_{y} \cos \left({ }^{t} \theta_{y}\right)\right)}{\partial^{0} y}-\frac{\partial\left(\underline{\theta}_{x} \cos \left({ }^{t} \theta_{x}\right)\right)}{\partial^{0} x}\right) \\
+2 \frac{\partial\left(\sin ^{t} \theta_{x}\right)}{\partial^{0} y} \frac{\partial\left(\underline{\theta}_{y} \cos \left({ }^{t} \theta_{y}\right)\right)}{\partial^{0} x}+2 \frac{\partial\left(\sin ^{t} \theta_{y}\right)}{\partial^{0} x} \frac{\partial\left(\underline{\theta}_{x} \cos \left({ }^{t} \theta_{x}\right)\right)}{\partial^{0} y}
\end{array}\right]
\end{array}\right]
\end{aligned}
$$

where $\bar{W}_{i p}^{N L}, \bar{W}_{s h}^{N L}$ and $\bar{W}_{b}^{N L}$ represent the strain energy per unit area for in-plane, shear, and bending deformations, respectively.

According to Barut [64], the strain energy per unit area for bending deformation given in Eq. (35d) can be further simplified as

$$
\bar{W}_{b}^{N L}=\frac{E h^{3}}{12\left(1-v^{2}\right)}\left[\begin{array}{l}
\left(-{ }_{0}^{t} \theta_{y, x}+{ }_{0}^{t} \theta_{x, y}\right)\left(-{ }_{0} \underline{\theta}_{y, x}+{ }_{0} \underline{\theta}_{x, y}\right) \\
+\frac{1-v}{2}\left[\left({ }_{0}^{t} \theta_{y, y}-{ }_{0}^{t} \theta_{x, x}\right)\left({ }_{0} \underline{\theta}_{y, y}-{ }_{0} \underline{\theta}_{x, x}\right)+2{ }_{0}^{t} \theta_{x, y}\left({ }_{0} \underline{\theta}_{y, x}\right)+2{ }_{0}^{t} \theta_{y, x}\left({ }_{0} \underline{\theta}_{x, y}\right)\right]
\end{array}\right]
$$

which can be rewritten as

$$
\bar{W}_{b}^{N L}=\frac{E h^{3}}{12\left(1-v^{2}\right)}\left[\left({ }_{0}^{t} \vartheta_{b}\right)\left({ }_{0} \underline{\vartheta}_{b}\right)+\frac{1-v}{2}\left[\begin{array}{l}
\left({ }_{0}^{t} \theta_{y, y}-{ }_{0}^{t} \theta_{x, x}\right)\left({ }_{0} \underline{\theta}_{y, y}-{ }_{0} \underline{\theta}_{x, x}\right) \\
+2{ }_{0}^{t} \theta_{x, y}\left({ }_{0} \underline{\theta}_{y, x}\right)+2{ }_{0}^{t} \theta_{y, x}\left({ }_{0} \underline{\theta}_{x, y}\right)
\end{array}\right]\right]
$$

with

$$
\begin{aligned}
& { }_{0}^{t} \vartheta_{b}=-{ }_{0}^{t} \theta_{y, x}+{ }_{0}^{t} \theta_{x, y} \\
& { }_{0} \underline{\vartheta}_{b}=-{ }_{0} \underline{\theta}_{y, x}+{ }_{0} \underline{\theta}_{x, y}
\end{aligned}
$$

where

$$
\begin{aligned}
& { }_{0} \underline{\theta}_{x, x}=\frac{\partial \underline{\theta}_{x}}{\partial^{0} x} ;{ }_{0} \underline{\theta}_{x, y}=\frac{\partial \underline{\theta}_{x}}{\partial^{0} y} \\
& { }_{0} \underline{\theta}_{y, x}=\frac{\partial \underline{\theta}_{y}}{\partial^{0} x} ;{ }_{0} \underline{\theta}_{y, y}=\frac{\partial \underline{\theta}_{y}}{\partial^{0} y}
\end{aligned}
$$




\section{Nonlinear kinematics of plate in peridynamics}

In peridynamics, the motion of a material point is influenced by the collective deformations of the surrounding material points which are called its family members [4, 6-8]. The original PD equation of motion is proposed by [4, 7]. For nonlinear analysis of a plate, the PD equation of motion for material point $k$ can be described in the discrete form as

$\rho_{(k)}\left({ }^{t} \ddot{\mathbf{u}}_{(k)}\right)=\sum_{j=1}^{N}\left(\begin{array}{c}{ }_{0}^{t} \overline{\mathbf{t}}_{(k)(j)}\left({ }^{t} \mathbf{u}_{(j)}-{ }^{t} \mathbf{u}_{(k)}, \underline{\mathbf{u}}_{(j)}-\underline{\mathbf{u}}_{(k)},{ }^{0} \mathbf{x}_{(j)}-{ }^{0} \mathbf{x}_{(k)}, t\right) \\ -{ }_{0}^{t} \overline{\mathbf{t}}_{(j)(k)}\left({ }^{t} \mathbf{u}_{(k)}-{ }^{t} \mathbf{u}_{(j)}, \underline{\mathbf{u}}_{(k)}-\underline{\mathbf{u}}_{(j)},{ }^{0} \mathbf{x}_{(k)}-{ }^{0} \mathbf{x}_{(j)}, t\right)\end{array}\right){ }^{0} V_{(j)}+{ }^{t} \overline{\mathbf{b}}_{(k)}$

where $\rho$ represents the mass density, ${ }^{t} \ddot{\mathbf{u}}$ represents the vector of accelerations at time $t$. The term, ${ }^{t} \mathbf{u}$ represents the vector of displacements at time $t$. Meanwhile, $\underline{\mathbf{u}}$ represents the vector of incremental displacement from time $t$ to time $t+\Delta t$. The term ${ }^{t} \overline{\mathbf{b}}_{(k)}$ represents the vector of external forces and moments per unit area at time $t$. The terms ${ }_{0}^{t} \overline{\mathbf{t}}_{(k)(j)}$ and ${ }_{0}^{t} \overline{\mathbf{t}}_{(j)(k)}$ represent the vectors of force densities at time $t$ in which ${ }_{0}^{t} \overline{\mathbf{t}}_{(k)(j)}$ is the force density that material point $j$ exerts on material point $k$. On the other hand, ${ }_{0}^{t} \overline{\mathbf{t}}_{(j)(k)}$ is the force density that material point $k$ exerts on material point $j$. Both of these force densities are measured with respect to the initial configuration. For a plate with five degrees of freedom, these force densities can be represented as

$$
\begin{aligned}
& { }_{0}^{t} \overline{\mathbf{t}}_{(k)(j)}=\left[\begin{array}{lllll}
{ }_{0}^{t} \bar{t}_{(k)(j)}^{u} & { }_{0}^{t} \bar{t}_{(k)(j)}^{v} & { }_{0}^{t} \bar{t}_{(k)(j)}^{w} & { }_{0}^{t} \bar{t}_{(k)(j)}^{\theta_{x}} & { }_{0}^{t} \bar{t}_{(k)(j)}^{\theta_{y}}
\end{array}\right]^{T} \\
& { }_{0}^{t} \overline{\mathbf{t}}_{(j)(k)}=\left[\begin{array}{lllll}
{ }_{0}^{t} \bar{t}_{(j)(k)}^{u} & { }_{0}^{t} \bar{t}_{(j)(k)}^{v} & { }_{0}^{t} \bar{t}_{(j)(k)}^{w} & { }_{0}^{t} \bar{t}_{(j)(k)}^{\theta_{x}} & { }_{0}^{t} \bar{t}_{(j)(k)}^{\theta_{y}}
\end{array}\right]^{T}
\end{aligned}
$$

where ${ }_{0}^{t} \bar{t}_{(k)(j)}^{u},{ }_{0}^{t} \bar{t}_{(k)(j)}^{v},{ }_{0}^{t} \bar{t}_{(k)(j)}^{w},{ }_{0}^{t} \bar{t}_{(k)(j)}^{\theta_{x}},{ }_{0}^{t} \bar{t}_{(k)(j)}^{\theta_{y}}$ represents force densities corresponding to five degrees of freedom, $u, v, w, \theta_{x}, \theta_{y}$, respectively. The demonstration of these force densities are shown in Fig. 5.

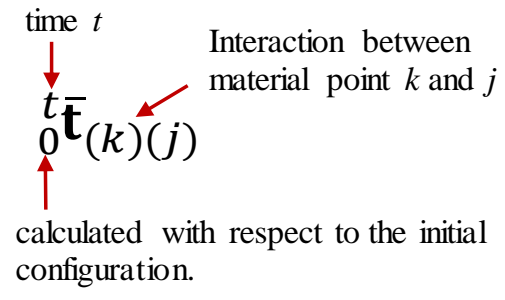

(a)

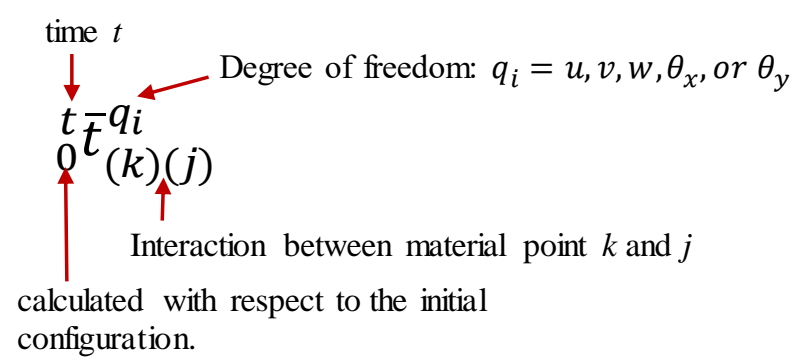

(b)

Fig. 5. Demonstration of force density at time $t$, (a): force density vector, (b): force density component

In Fig. 5, the left superscript " $t$ " represents the current time. Meanwhile, the left subscript " 0 " represents that the force density is calculated with respect to the initial configuration. The right subscript " $(j)(k)$ " represents the interaction between material points $j$ and $k$. The parameter $q_{i}$ represents the degree of freedom. 
As proposed in [8] and later used in [14, 27], the relationships between the PD force densities and the strain energy per unit area can be represented as

$$
{ }_{0}^{t} \bar{t}_{(k)(j)}^{q_{i}}=-\frac{1}{{ }^{0} V_{(j)}} \frac{\partial \bar{W}_{(k)}^{N L P D}}{\partial \underline{q}_{i(k)}}
$$

and

$$
{ }_{0}^{t} \bar{t}_{(j)(k)}^{q_{i}}=-\frac{1}{{ }^{0} V_{(k)}} \frac{\partial \bar{W}_{(j)}^{N L P D}}{\partial \underline{q}_{i(j)}}
$$

with

$$
\begin{aligned}
& q_{i}=u, v, w, \theta_{x}, \theta_{y} \\
& \underline{q}_{i}=\underline{u}, \underline{v}, \underline{w}, \underline{\theta}_{x}, \underline{\theta}_{y}
\end{aligned}
$$

where ${ }_{0}^{t} \bar{t}_{(k)(j)}^{q_{i}}$ and ${ }_{0}^{t} \bar{t}_{(j)(k)}^{q_{i}}$ represent the force density component that corresponds to the degree of freedom $q_{i}$ as given in Eq. (38). The parameter $q_{i}$ represents the degree of freedom which can be $u, v, w, \theta_{x}$ or $\theta_{y}$. The parameter $\underline{q}_{i}$ represents the incremental value of $q_{i}$ which can be $\underline{u}, \underline{v}, \underline{w}, \underline{\theta}_{x}$, or $\underline{\theta}_{y}$.

In the following sections, first, nonlinear strain energy per unit area for a plate in peridynamics is presented in Section 4.1. The PD constants are obtained by comparing the nonlinear strain energies per unit area in PD with those in classical continuum mechanics. Later, the PD equations of motion for geometrically nonlinear analysis of a plate are presented in Section 4.2.

\subsection{Peridynamic strain energy per unit area}

Similar to the classical formulation of the nonlinear strain energy per unit area given in Eq. (35), the nonlinear strain energy per unit area in PD for a plate can be decomposed as

$$
\bar{W}_{(k)}^{N L P D}=\bar{W}_{i p(k)}^{N L P D}+\bar{W}_{s h(k)}^{N L P D}+\bar{W}_{b(k)}^{N L P D}
$$

where $\bar{W}_{i p(k)}^{N L P D}, \bar{W}_{s h(k)}^{N L P D}$, and $\bar{W}_{b(k)}^{N L P D}$ represent the nonlinear PD strain energies per unit area for the in-plane, shear, and bending deformations, respectively.

The nonlinear PD strain energy per unit area for the in-plane deformations can be represented as

$$
\bar{W}_{i p(k)}^{N L P D}=2 a_{i p}\left({ }_{0}^{t} \vartheta_{(k)}\right)\left({ }_{0} \underline{\vartheta}_{(k)}\right)+2 b_{i p} \sum_{j=1}^{N}\left({ }_{0}^{t} s_{i p(k)(j)}\right)\left({ }_{0} \underline{S}_{i p(k)(j)}\right){ }^{0} \xi_{(k)(j)}{ }^{0} V_{(j)}
$$

with

$$
\begin{aligned}
{ }_{0}^{t} s_{i p(k)(j)}= & \frac{\left({ }^{t} u_{(j)}-{ }^{t} u_{(k)}\right) \cos \varphi+\left({ }^{t} v_{(j)}-{ }^{t} v_{(k)}\right) \sin \varphi}{{ }^{0} \xi_{(k)(j)}} \\
& +\frac{1}{2} \frac{\left({ }^{t} u_{(j)}-{ }^{t} u_{(k)}\right)^{2}+\left({ }^{t} v_{(j)}-{ }^{t} v_{(k)}\right){ }^{2}+\left({ }^{t} w_{(j)}-{ }^{t} w_{(k)}\right)^{2}}{{ }^{0} \xi_{(k)(j)}^{2}}
\end{aligned}
$$




$$
\begin{aligned}
& { }_{0} \underline{S}_{i p(k)(j)}=\frac{\left(\underline{u}_{(j)}-\underline{u}_{(k)}\right) \cos \varphi+\left(\underline{v}_{(j)}-\underline{v}_{(k)}\right) \sin \varphi}{{ }^{0} \xi_{(k)(j)}}+\frac{\left({ }^{t} u_{(j)}-{ }^{t} u_{(k)}\right)\left(\underline{u}_{(j)}-\underline{u}_{(k)}\right)}{\hat{\xi}_{(k)(j)}^{2}} \\
& +\frac{\left({ }^{t} v_{(j)}-{ }^{t} v_{(k)}\right)\left(\underline{v}_{(j)}-\underline{v}_{(k)}\right)}{{ }^{0} \xi_{(k)(j)}^{2}}+\frac{\left({ }^{t} w_{(j)}-{ }^{t} w_{(k)}\right)\left(\underline{w}_{(j)}-\underline{w}_{(k)}\right)}{\xi_{(k)(j)}^{2}} \\
& { }_{0}^{t} \vartheta_{(k)}=d_{i p} \sum_{j=1}^{N}{ }_{0}^{t} s_{i p(k)(j)}{ }^{0} V_{(j)} \\
& { }_{0} \underline{\vartheta}_{(k)}=d_{i p} \sum_{j=1}^{N}\left({ }_{0} \underline{S}_{i p(k)(j)}\right)^{0} V_{(j)} \\
& { }^{0} V_{(j)}={ }^{0} A_{(j)} h
\end{aligned}
$$

In Eq. (41), $k$ is a material point in the PD discretized model and $j$ is a family member of material point $k$ as shown in Fig. 6 . The parameter $N$ represents the total number of family members of material point $k$. The term ${ }_{0}^{t} s_{i p(k)(j)}$ given in Eq. (41b) represents the nonlinear bond stretch at time $t$. Meanwhile, ${ }_{0} \underline{S}_{i p(k)(j)}$ given in Eq. (41c) represents the incremental bond stretch from time $t$ to time $t+\Delta t$. Both bond stretches are measured with respect to the initial configuration. The term ${ }_{0}^{t} \vartheta_{(k)}$ given in Eq. (41d) represents the dilatation at time $t$. The classical formulation of this dilatation is given in Eq. (34d). The term ${ }_{0} \underline{\vartheta}_{(k)}$ given in Eq. (41e) represents the incremental dilatation from time $t$ to time $t+\Delta t$. The classical formulation of this incremental dilatation is given in Eq. (34e).

In Eq. (41), the terms $a_{i p}, b_{i p}, d_{i p}$ represent the PD constants for in-plane deformations. The terms ${ }^{t} u_{(k)},{ }^{t} v_{(k)}$ and ${ }^{t} w_{(k)}$ represent the displacements at time $t$ of material point $k$. Meanwhile, $\underline{u}_{(k)}, \underline{v}_{(k)}$ and $\underline{w}_{(k)}$ represent the incremental displacements of material point $k$ from time $t$ to time $t+\Delta t$. Similarly, ${ }^{t} u_{(j)},{ }^{t} v_{(j)}$ and ${ }^{t} w_{(j)}$ represent the displacements at time $t$ of material point $j$. Meanwhile, $\underline{u}_{(j)}, \underline{v}_{(j)}$ and $\underline{w}_{(j)}$ represent the incremental displacements of material point $j$ from time $t$ to time $t+\Delta t$. These displacements and incremental displacements are shown in Fig. 4. The term ${ }^{0} V_{(j)}$ represents the volume of material point $j$ in the undeformed configuration (at time $t=0),{ }^{0} A_{(j)}$ represents the area of material point $j$ in the undeformed configuration. The term ${ }^{0} \xi_{(k)(j)}$ represents the distance between material points $k$ and $j$ in the undeformed configuration as shown in Fig. 6 which is defined as

$$
{ }^{0} \xi_{(k)(j)}=\sqrt{\left({ }^{0} x_{(j)}-{ }^{0} x_{(k)}\right)^{2}+\left({ }^{0} y_{(j)}-{ }^{0} y_{(k)}\right)^{2}}
$$

In Eq. (41), $\varphi$ represents the angle between the ${ }^{0} x$ axis in the undeformed configuration and the line connecting material points $k$ and $j$ as shown in Fig. 3 and Fig. 6. The terms $\sin \varphi$ and $\cos \varphi$ in Eq. (41) can be calculated as

$$
\cos \varphi=\frac{{ }^{0} x_{(j)}-{ }^{0} x_{(k)}}{{ }^{0} \xi_{(k)(j)}} ; \quad \sin \varphi=\frac{{ }^{0} y_{(j)}-{ }^{0} y_{(k)}}{{ }^{0} \xi_{(k)(j)}}
$$




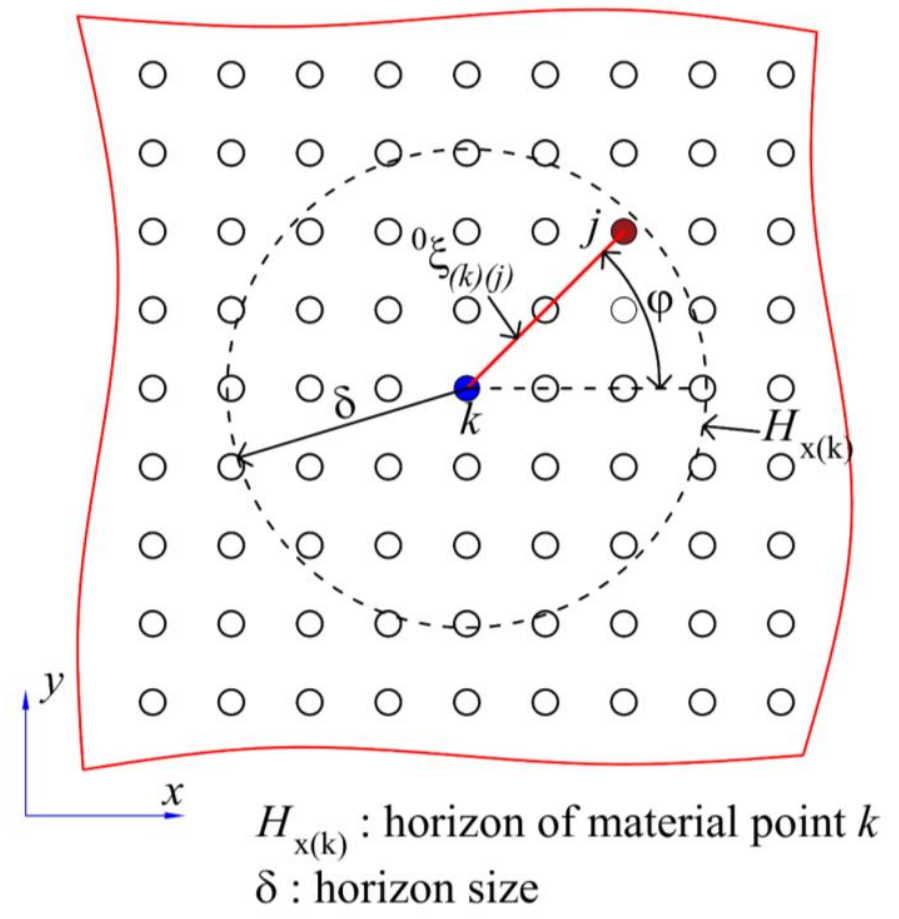

Fig. 6. Material point and its family members

The PD constants for the in-plane deformations, $a_{i p}, b_{i p}, d_{i p}$ can be obtained by comparing the strain energy per unit area for in-plane deformations in PD to those in classical continuum mechanics as presented in Appendix A. These PD constants can be represented as

$$
\begin{aligned}
& d_{i p}=\frac{2}{\pi h \delta^{2}} \\
& a_{i p}=\frac{E h(3 v-1)}{4\left(1-v^{2}\right)} \\
& b_{i p}=\frac{3 E}{(1+v) \pi \delta^{3}}
\end{aligned}
$$

where $\delta$ represents the horizon size. The terms $E$ and $v$ represent the elastic modulus and Poisson's ratio of material, $h$ represents the thickness of the plate.

Based on the classical formulation given in Eq. (35c), the nonlinear PD strain energy per unit area for the shear deformations given in Eq. (40) can be presented as

$$
\bar{W}_{s h(k)}^{N L P D}=\frac{1}{2} C_{s h} \sum_{j=1}^{N}\left(\frac{{ }^{t} w_{(j)}-{ }^{t} w_{(k)}}{{ }^{0} \xi_{(k)(j)}}-\frac{{ }^{t} \hat{\theta}_{(k)}+{ }^{t} \hat{\theta}_{(j)}}{2}\right)\left(\frac{\underline{w}_{(j)}-\underline{w}_{(k)}}{{ }^{0} \xi_{(k)(j)}}-\frac{\underline{\hat{\theta}}_{(k)}+\underline{\hat{\theta}}_{(j)}}{2}\right){ }^{0} \xi_{(k)(j)}{ }^{0} V_{(j)}
$$

with

$$
\begin{aligned}
& { }^{t} \hat{\theta}_{(k)}=-\left(\sin ^{t} \theta_{y(k)}\right) \cos \varphi+\left(\sin ^{t} \theta_{x(k)}\right) \sin \varphi \\
& \underline{\hat{\theta}}_{(k)}=-\underline{\theta}_{y(k)} \cos \left({ }^{t} \theta_{y(k)}\right) \cos \varphi+\underline{\theta}_{x(k)} \cos \left({ }^{t} \theta_{x(k)}\right) \sin \varphi
\end{aligned}
$$


${ }^{t} \hat{\theta}_{(j)}=-\left(\sin ^{t} \theta_{y(j)}\right) \cos \varphi+\left(\sin ^{t} \theta_{x(j)}\right) \sin \varphi$

$\underline{\hat{\theta}}_{(j)}=-\underline{\theta}_{y(j)} \cos \left({ }^{t} \theta_{y(j)}\right) \cos \varphi+\underline{\theta}_{x(j)} \cos \left({ }^{t} \theta_{x(j)}\right) \sin \varphi$

where ${ }^{t} \theta_{x(k)}$ and ${ }^{t} \theta_{y(k)}$ represent the rotations of material point $k$ at time $t$. The terms $\underline{\theta}_{x(k)}$ and $\underline{\theta}_{y(k)}$ represent the incremental rotations from time $t$ to time $t+\Delta t$ of material point $k$ (see Fig. 3 and Fig. 7). Similarly, ${ }^{t} \theta_{x(j)}$ and ${ }^{t} \theta_{y(j)}$ represent the rotations of material point $j$ at time $t$. The terms $\underline{\theta}_{x(j)}$ and $\underline{\theta}_{y(j)}$ represent the incremental rotations from time $t$ to time $t+\Delta t$ of material point $j$ (see Fig. 3 and Fig. 7). The parameter $C_{s h}$ represents the PD constant for shear deformations. As presented in Appendix B, $C_{s h}$ is determined by comparing the strain energy per unit area for shear deformations in PD to those in $\mathrm{CCM}$ as

$C_{s h}=\frac{3 k_{s} E}{(1+v) \pi \delta^{3}}$

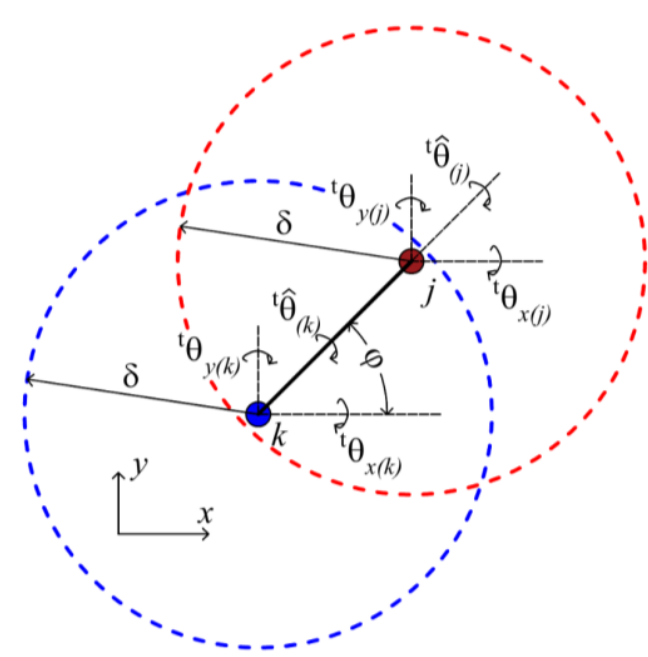

(a)

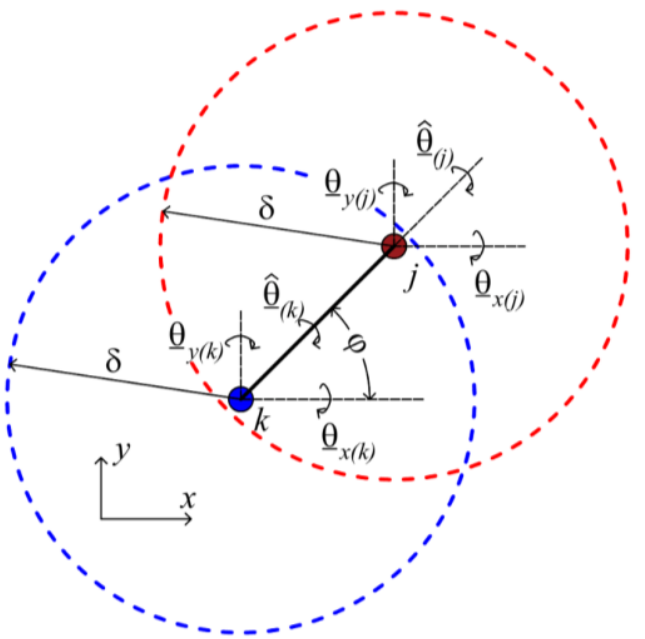

(b)

Fig. 7. Rotations and incremental rotations of material points (a): rotations at time $t$, (b): incremental rotations from time $t$ to time $t+\Delta t$

Based on the classical formulation given in Eq. (35d), the nonlinear PD strain energy per unit area for the bending deformations given in Eq. (40) can be presented as

$$
\bar{W}_{b(k)}^{N L P D}=2 a_{b}\left({ }_{0}^{t} \vartheta_{b(k)}\right)\left({ }_{0} \underline{\vartheta}_{b(k)}\right)+2 b_{b} \sum_{j=1}^{N}\left({ }_{0}^{t} s_{b(k)(j)}\right)\left({ }_{0} \underline{s}_{b(k)(j)}\right){ }^{0} \xi_{(k)(j)}{ }^{0} V_{(j)}
$$

with

$$
\begin{aligned}
& { }_{0}^{t} \vartheta_{b(k)}=d_{b} \sum_{j=1}^{N}{ }_{0}^{t} S_{b(k)(j)}{ }^{0} V_{(j)} \\
& { }_{0} \underline{\vartheta}_{b(k)}=d_{b} \sum_{j=1}^{N}\left({ }_{0} \underline{S}_{b(k)(j)}\right){ }^{0} V_{(j)}
\end{aligned}
$$


${ }_{0}^{t} s_{b(k)(j)}=\frac{-\left({ }^{t} \theta_{y(j)}-{ }^{t} \theta_{y(k)}\right) \cos \varphi+\left({ }^{t} \theta_{x(j)}-{ }^{t} \theta_{x(k)}\right) \sin \varphi}{{ }^{0} \xi_{(k)(j)}}$

${ }_{0} \underline{S}_{b(k)(j)}=\frac{-\left(\underline{\theta}_{y(j)}-\underline{\theta}_{y(k)}\right) \cos \varphi+\left(\underline{\theta}_{x(j)}-\underline{\theta}_{x(k)}\right) \sin \varphi}{{ }^{0} \xi_{(k)(j)}}$

where ${ }_{0}^{t} s_{b(k)(j)}$ represents the bond stretch for bending deformations at time $t$. Meanwhile, ${ }_{0} \underline{S}_{b(k)(j)}$ represents the incremental bond stretch for bending deformation from time $t$ to time $t+\Delta t$. The terms ${ }^{t} \theta_{x(j)}$ and ${ }^{t} \theta_{y(j)}$ represent the rotations at time $t$ of material point $j$. Meanwhile, $\underline{\theta}_{x(j)}$ and $\underline{\theta}_{y(j)}$ represent the incremental rotations from time $t$ to time $t+\Delta t$ of material point $j$. The term ${ }_{0}^{t} \vartheta_{b(k)}$ and ${ }_{0} \underline{\vartheta}_{b(k)}$ given in Eqs. (47b-c) corresponds to the term ${ }_{0}^{t} \vartheta_{b}$ and ${ }_{0} \underline{\vartheta}_{b}$ in classical continuum mechanics given in Eqs. (36c-d).

In Eq. (47), the terms $a_{b}, b_{b}, d_{b}$ represent the PD constants for bending deformations. As presented in Appendix C, these PD constants can be represented as

$$
\begin{aligned}
& d_{b}=\frac{2}{\pi h \delta^{2}} \\
& a_{b}=\frac{E h^{3}(3 v-1)}{48\left(1-v^{2}\right)} \\
& b_{b}=\frac{E h^{2}}{4(1+v) \pi \delta^{3}}
\end{aligned}
$$

\subsection{Nonlinear peridynamic equations of motion for a plate}

By substituting the strain energy per unit area components given in Eq. (41), Eq. (45), and Eq. (47) into Eq. (40), the strain energy per unit area for a material point $k$ in the plate is calculated. Next, by substituting the strain energy per unit area given in Eq. (40) into Eq. (39a), the force density ${ }_{0}^{t} \bar{t}_{(k)(j)}^{q_{i}}$ can be obtained as

$$
\begin{aligned}
& { }_{0}^{t} \bar{t}_{(k)(j)}^{u}=\left[\frac{2 a_{i p} d_{i p}}{{ }^{0} \xi_{(k)(j)}}{ }_{0}^{t} \vartheta_{(k)}+2 b_{i p 0}{ }^{t} s_{i p(k)(j)}\right]\left(\cos \varphi+\frac{{ }^{t} u_{(j)}-{ }^{t} u_{(k)}}{{ }^{0} \xi_{(k)(j)}}\right) \\
& { }_{0}^{t} \bar{t}_{(k)(j)}^{v}=\left[\frac{2 a_{i p} d_{i p}}{{ }^{0} \xi_{(k)(j)}}{ }_{0}^{t} \vartheta_{(k)}+2 b_{i p}{ }_{0}^{t} s_{i p(k)(j)}\right]\left(\sin \varphi+\frac{{ }^{t} v_{(j)}-{ }^{t} v_{(k)}}{{ }^{0} \xi_{(k)(j)}}\right) \\
& { }_{0}^{t} \bar{t}_{(k)(j)}^{w}=\left(\frac{2 a_{i p} d_{i p}}{{ }^{0} \xi_{(k)(j)}}{ }_{0}^{t} \vartheta_{(k)}+2 b_{i p}{ }_{0}^{t} s_{i p(k)(j)}\right) \frac{{ }^{t} w_{(j)}-{ }^{t} w_{(k)}}{{ }^{0} \xi_{(k)(j)}}+\frac{1}{2} C_{s}\left(\frac{{ }^{t} w_{(j)}-{ }^{t} w_{(k)}}{{ }^{0} \xi_{(k)(j)}}-\frac{{ }^{t} \hat{\theta}_{(k)}+{ }^{t} \hat{\theta}_{(j)}}{2}\right) \\
& { }_{0}^{t} \bar{t}_{(k)(j)}=\left[\frac{2 a_{b} d_{b}}{{ }^{0} \xi_{(k)(j)}}{ }_{0}^{t} \vartheta_{b(k)}+2 b_{b}{ }_{0}^{t} s_{b(k)(j)}+\frac{C_{s}{ }^{0} \xi_{(k)(j)}}{2}\left(\frac{{ }^{t} w_{(j)}-{ }^{t} w_{(k)}}{{ }^{0} \xi_{(k)(j)}}-\frac{{ }^{t} \hat{\theta}_{(k)}+{ }^{t} \hat{\theta}_{(j)}}{2}\right) \frac{\cos \left({ }^{t} \theta_{x(k)}\right)}{2}\right] \sin \varphi(49
\end{aligned}
$$


${ }_{0}^{t} \bar{t}_{(k)(j)}^{\theta_{y}}=-\left[\frac{2 a_{b} d_{b}}{{ }^{0} \xi_{(k)(j)}}{ }_{0}^{t} \vartheta_{b(k)}+2 b_{b 0}{ }^{t} s_{b(k)(j)}+\frac{C_{s}{ }^{0} \xi_{(k)(j)}}{2}\left(\frac{{ }^{t} w_{(j)}-{ }^{t} w_{(k)}}{{ }^{0} \xi_{(k)(j)}}-\frac{{ }^{t} \hat{\theta}_{(k)}+{ }^{t} \hat{\theta}_{(j)}}{2}\right) \frac{\cos \left({ }^{t} \theta_{y(k)}\right)}{2}\right] \cos \varphi$

Similarly, by considering material point $j$ and its family members within its horizon size, the strain energy per unit area of material point $j$ can be calculated using Eqs. (40-47). Therefore, the force density ${ }_{0}^{t} \bar{t}_{(j)(k)}^{q_{i}}$ that material point $k$ exerts on material point $j$ can be obtained using Eq. (39b) as

$$
\begin{aligned}
& { }_{0}^{t} \bar{t}_{(j)(k)}^{u}=-\left[\frac{2 a_{i p} d_{i p}}{{ }^{0} \xi_{(k)(j)}}{ }_{0}^{t} \vartheta_{(j)}+2 b_{i p 0}{ }_{0}^{t} s_{i p(k)(j)}\right]\left(\cos \varphi+\frac{{ }^{t} u_{(j)}-{ }^{t} u_{(k)}}{{ }^{0} \xi_{(k)(j)}}\right) \\
& { }_{0}^{t} \bar{t}_{(j)(k)}^{v}=-\left[\frac{2 a_{i p} d_{i p}}{{ }^{0} \xi_{(k)(j)}}{ }_{0}^{t} \vartheta_{(j)}+2 b_{i p ~}{ }_{0}^{t} s_{i p(k)(j)}\right]\left(\sin \varphi+\frac{{ }^{t} v_{(j)}-{ }^{t} v_{(k)}}{{ }^{0} \xi_{(k)(j)}}\right) \\
& { }_{0}^{t} \bar{t}_{(j)(k)}^{w}=-\left(\frac{2 a_{i p} d_{i p}}{{ }^{0} \xi_{(k)(j)}}{ }_{0}^{t} \vartheta_{(j)}+2 b_{i p 0}{ }_{0}^{t} s_{i p(k)(j)}\right) \frac{{ }^{t} w_{(j)}-{ }^{t} w_{(k)}}{{ }^{0} \xi_{(k)(j)}}-\frac{1}{2} C_{s}\left(\frac{{ }^{t} w_{(j)}-{ }^{t} w_{(k)}}{{ }^{0} \xi_{(k)(j)}}-\frac{{ }^{t} \hat{\theta}_{(k)}+{ }^{t} \hat{\theta}_{(j)}}{2}\right) \\
& { }_{0}^{t} \bar{t}_{(j)(k)} \theta_{x}=-\left[\begin{array}{l}
\frac{2 a_{b} d_{b}}{{ }^{0} \xi_{(k)(j)}}{ }_{0}^{t} \vartheta_{b(j)}+2 b_{b}{ }_{0}^{t} s_{b(k)(j)} \\
+\frac{C_{s}{ }^{0} \xi_{(k)(j)}}{2}\left(\frac{{ }^{t} w_{(j)}-{ }^{t} w_{(k)}}{{ }^{0} \xi_{(k)(j)}}-\frac{{ }^{t} \hat{\theta}_{(k)}+{ }^{t} \hat{\theta}_{(j)}}{2}\right) \frac{\cos \left({ }^{t} \theta_{x(j)}\right)}{2}
\end{array}\right] \sin \varphi \\
& { }_{0}^{t} \bar{t}_{(j)(k)}^{\theta_{y}}=\left[\begin{array}{l}
\frac{2 a_{b} d_{b}}{{ }^{0} \xi_{(k)(j)}}{ }_{0}^{t} \vartheta_{b(j)}+2 b_{b 0}{ }_{0}^{t} s_{b(k)(j)} \\
+\frac{C_{s}{ }^{0} \xi_{(k)(j)}}{2}\left(\frac{{ }^{t} w_{(j)}-{ }^{t} w_{(k)}}{{ }^{0} \xi}-\frac{{ }^{t} \hat{\theta}_{(k)}+{ }^{t} \hat{\theta}_{(j)}}{2}\right) \frac{\cos \left({ }^{t} \theta_{y(j)}\right)}{2}
\end{array}\right] \cos \varphi
\end{aligned}
$$

Therefore, by substituting the force densities given in Eqs. (49-50) into Eq. (37), the nonlinear PD equations of motion for a plate can be rewritten as

$$
\begin{aligned}
& \rho h\left({ }^{t} \ddot{u}_{(k)}\right)=\sum_{j=1}^{N}\left[\frac{2 a_{i p} d_{i p}}{{ }^{0} \xi_{(k)(j)}}\left({ }_{0}^{t} \vartheta_{(k)}+{ }_{0}^{t} \vartheta_{(j)}\right)+4 b_{i p 0}{ }^{t} s_{i p(k)(j)}\right]\left(\cos \varphi+\frac{{ }^{t} u_{(j)}-{ }^{t} u_{(k)}}{{ }^{0} \xi_{(k)(j)}}\right){ }^{0} V_{(j)}+{ }^{t} \bar{b}_{x(k)} \\
& \rho h\left(\ddot{v}_{(k)}\right)=\sum_{j=1}^{N}\left[\frac{2 a_{i p} d_{i p}}{{ }^{0} \xi_{(k)(j)}}\left({ }_{0}^{t} \vartheta_{(k)}+{ }_{0}^{t} \vartheta_{(j)}\right)+4 b_{i p 0}{ }^{t} s_{i p(k)(j)}\right]\left(\sin \varphi+\frac{{ }^{t} v_{(j)}-{ }^{t} v_{(k)}}{{ }^{0} \xi_{(k)(j)}}\right){ }^{0} V_{(j)}+{ }^{t} \bar{b}_{y(k)}
\end{aligned}
$$

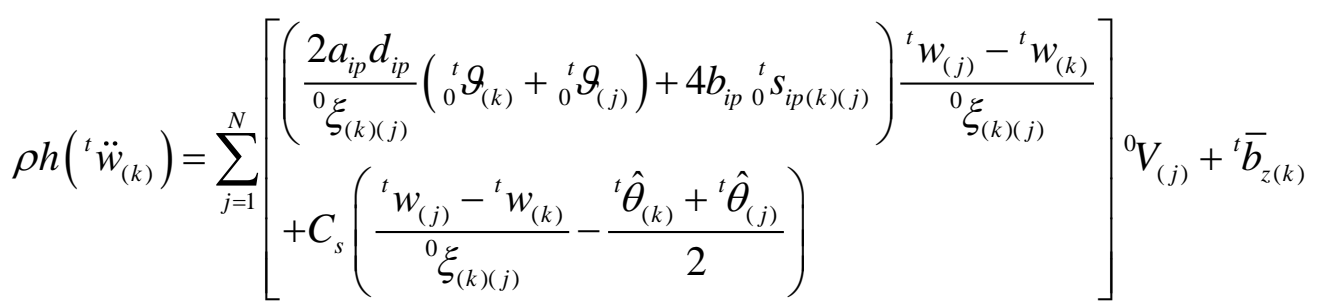




$$
\begin{aligned}
& \frac{\rho h^{3}}{12}\left({ }^{t} \ddot{\theta}_{x(k)}\right)=\sum_{j=1}^{N}\left[\begin{array}{l}
\frac{2 a_{b} d_{b}}{{ }^{0} \xi_{(k)(j)}}\left({ }_{0}^{t} \vartheta_{b(k)}+{ }_{0}^{t} \vartheta_{b(j)}\right)+4 b_{b 0}{ }_{0}^{t} s_{b(k)(j)} \\
+\frac{C_{s}{ }^{0} \xi_{(k)(j)}}{2}\left(\frac{{ }^{t} w_{(j)}-{ }^{t} w_{(k)}}{{ }^{0} \xi_{(k)(j)}}-\frac{{ }^{t} \hat{\theta}_{(k)}+{ }^{t} \hat{\theta}_{(j)}}{2}\right) \frac{\cos \left({ }^{t} \theta_{x(k)}\right)+\cos \left({ }^{t} \theta_{x(j)}\right)}{2}
\end{array}\right] \sin \varphi^{0} V_{(j)} \\
& +{ }^{t} \bar{m}_{x(k)} \\
& \frac{\rho h^{3}}{12}\left({ }^{t} \ddot{\theta}_{y(k)}\right)=-\sum_{j=1}^{N}\left[\begin{array}{l}
\frac{2 a_{b} d_{b}}{{ }^{0} \xi_{(k)(j)}}\left({ }_{0}^{t} \vartheta_{b(k)}+{ }_{0}^{t} \vartheta_{b(j)}\right)+4 b_{b}{ }_{0}^{t} s_{b(k)(j)} \\
+\frac{C_{s}{ }^{0} \xi_{(k)(j)}}{2}\left(\frac{{ }^{t} w_{(j)}-{ }^{t} w_{(k)}}{{ }^{0} \xi_{(k)(j)}}-\frac{{ }^{t} \hat{\theta}_{(k)}+{ }^{t} \hat{\theta}_{(j)}}{2}\right) \frac{\cos \left({ }^{t} \theta_{y(k)}\right)+\cos \left({ }^{t} \theta_{y(j)}\right)}{2}
\end{array}\right] \cos \varphi^{0} V_{(j)} \\
& +{ }^{t} \bar{m}_{y(k)}
\end{aligned}
$$

where ${ }^{t} \bar{b}_{x(k)},{ }^{t} \bar{b}_{y(k)},{ }^{t} \bar{b}_{z(k)}$ represent external forces per unit area applied on material point $k$ at time $t,{ }^{t} \bar{m}_{x(k)}$ and ${ }^{t} \bar{m}_{y(k)}$ represent external moments per unit area applied on material point $k$ at time $t$. Note that, for small strain problems, the thickness of the plate and volume of material points are assumed to be unchanged. Therefore, the external loading at time $t$ can be represented as

$$
\begin{aligned}
& { }^{t} \bar{b}_{x(k)}=\frac{{ }^{t} F_{x(k)}}{{ }^{0} A_{(k)}} ; \quad{ }^{t} \bar{b}_{y(k)}=\frac{{ }^{t} F_{y(k)}}{{ }^{0} A_{(k)}} ; \quad{ }^{t} \bar{b}_{z(k)}=\frac{{ }^{t} F_{z(k)}}{{ }^{0} A_{(k)}} \\
& { }^{t} \bar{m}_{x(k)}=\frac{{ }^{t} M_{x(k)}}{{ }^{0} A_{(k)}} ; \quad{ }^{t} \bar{m}_{y(k)}=\frac{{ }^{t} M_{y(k)}}{{ }^{0} A_{(k)}}
\end{aligned}
$$

with

${ }^{0} A_{(k)}=\frac{{ }^{0} V_{(k)}}{h}$

where ${ }^{0} A_{(k)}$ represents the area of material point $k$ in the undeformed configuration. The terms ${ }^{t} F_{x(k)},{ }^{t} F_{y(k)}$ and ${ }^{t} F_{z(k)}$ represent the external forces applied on material point $k$ at time $t$. Meanwhile, ${ }^{t} M_{x(k)}$ and ${ }^{t} M_{y(k)}$ represent the external bending moments applied on material point $k$ at time $t$.

\section{Damage criteria}

Progressive damages can be involved in PD simulations by updating the state of each interaction. According to Silling and Askari [7], the state of the interaction, intact or broken, between material points $k$ and $j$ is represented by the damage parameter $\mu_{(k)(j)}$ which can be defined as

$$
\mu_{(k)(j)}=\left\{\begin{array}{lll}
1 & \Leftrightarrow & \text { interaction exists } \\
0 & \Leftrightarrow & \text { interaction is broken }
\end{array}\right.
$$


Therefore, by introducing a damage parameter, $\mu_{(k)(j)}$, the PD equations of motion given in Eq. (51) can be rewritten as [7]

$$
\overline{\mathbf{m}}_{(k)}\left({ }^{t} \ddot{\mathbf{u}}_{(k)}\right)=\sum_{j=1}^{N} \mu_{(k)(j)}{ }_{0}^{t} \overline{\mathbf{f}}_{(k)(j)}{ }^{0} V_{(j)}+{ }^{t} \overline{\mathbf{b}}_{(k)}
$$

or

$$
\overline{\mathbf{m}}_{(k)}\left({ }^{t} \ddot{\mathbf{u}}_{(k)}\right)={ }_{0}^{t} \overline{\mathbf{F}}_{(k)}+{ }^{t} \overline{\mathbf{b}}_{(k)}
$$

with

$$
\begin{aligned}
& \overline{\mathbf{m}}_{(k)}=\left[\begin{array}{ccccc}
\rho h & 0 & 0 & 0 & 0 \\
0 & \rho h & 0 & 0 & 0 \\
0 & 0 & \rho h & 0 & 0 \\
0 & 0 & 0 & \frac{\rho h^{3}}{12} & 0 \\
0 & 0 & 0 & 0 & \frac{\rho h^{3}}{12}
\end{array}\right] ;{ }^{t} \overline{\mathbf{b}}_{(k)}=\left[\begin{array}{c}
{ }^{t} \bar{b}_{x} \\
{ }^{t} \bar{b}_{y} \\
{ }^{t} \bar{b}_{z} \\
{ }^{t} \bar{m}_{x} \\
{ }^{t} \bar{m}_{y}
\end{array}\right] ;{ }_{0}^{t} \overline{\mathbf{f}}_{(k)(j)}=\left[\begin{array}{c}
t \\
0 \\
0 \\
{ }_{0}^{t} \bar{f}_{(k)(j)}^{v} \\
{ }^{t} \bar{f}_{(k)(j)}^{w} \\
{ }_{0}^{t} \bar{f}_{(k)(j)}^{\theta_{x}} \\
{ }_{0}^{t} \bar{f}_{(k)(j)}^{\theta_{y}}
\end{array}\right] \\
& { }_{0}^{t} \overline{\mathbf{F}}_{(k)}=\sum_{j=1}^{N} \mu_{(k)(j)}{ }_{0}^{t} \overline{\mathbf{f}}_{(k)(j)}{ }^{0} V_{(j)} \\
& { }_{0}^{t} \overline{\mathbf{f}}_{(k)(j)}={ }_{0}^{t} \overline{\mathbf{t}}_{(k)(j)}-{ }_{0}^{t} \overline{\mathbf{t}}_{(k)(j)}
\end{aligned}
$$

and

$$
\begin{aligned}
& { }_{0}^{t} \bar{f}_{(k)(j)}^{u}={ }_{0}^{t} \bar{t}_{(k)(j)}^{u}-{ }_{0}^{t} \bar{t}_{(j)(k)}^{u}=\left[\frac{2 a_{i p} d}{{ }^{0} \xi_{(k)(j)}}\left({ }_{0}^{t} \vartheta_{(k)}+{ }_{0}^{t} \vartheta_{(j)}\right)+4 b_{i p 0}{ }_{0}^{t} S_{i p(k)(j)}\right]\left(\cos \varphi+\frac{{ }^{t} u_{(j)}-{ }^{t} u_{(k)}}{{ }^{0} \xi_{(k)(j)}}\right) \\
& { }_{0}^{t} \bar{f}_{(k)(j)}^{v}={ }_{0}^{t} \bar{t}_{(k)(j)}^{v}-{ }_{0}^{t} \bar{t}_{(j)(k)}^{v}=\left[\frac{2 a_{i p} d_{i p}}{{ }^{0} \xi_{(k)(j)}}\left({ }_{0}^{t} \vartheta_{(k)}+{ }_{0}^{t} \vartheta_{(j)}\right)+4 b_{i p 0}{ }^{t} s_{i p(k)(j)}\right]\left(\sin \varphi+\frac{{ }^{t} v_{(j)}-{ }^{t} v_{(k)}}{{ }^{0} \xi_{(k)(j)}}\right) \\
& { }_{0}^{t} \bar{f}_{(k)(j)}^{w}={ }_{0}^{t} \bar{t}_{(k)(j)}^{w}-{ }_{0}^{t} \bar{t}_{(j)(k)}^{w}=\left(\frac{2 a_{i p} d}{{ }^{0} \xi_{(k)(j)}}\left({ }_{0}^{t} \vartheta_{(k)}+{ }_{0}^{t} \vartheta_{(j)}\right)+4 b_{i p 0}{ }_{0}^{t} S_{i p(k)(j)}\right) \frac{{ }^{t} w_{(j)}-{ }^{t} w_{(k)}}{{ }^{0} \xi_{(k)(j)}} \\
& +C_{s}\left(\frac{{ }^{t} w_{(j)}-{ }^{t} w_{(k)}}{{ }^{0} \xi_{(k)(j)}}-\frac{{ }^{t} \hat{\theta}_{(k)}+{ }^{t} \hat{\theta}_{(j)}}{2}\right)
\end{aligned}
$$

$$
\begin{aligned}
& { }_{0}^{t} \bar{f}_{(k)(j)}^{\theta_{x}}={ }_{0}^{t} \bar{t}_{(k)(j)}^{\theta_{x}}-{ }_{0}^{t} \bar{t}_{(j)(k)}^{\theta_{x}} \\
& =\left[\begin{array}{l}
\frac{2 a_{b} d_{b}}{{ }^{0} \xi_{(k)(j)}}\left({ }_{0}^{t} \vartheta_{b(k)}+{ }_{0}^{t} \vartheta_{b(j)}\right)+4 b_{b 0}{ }_{0}^{t} s_{b(k)(j)} \\
+\frac{C_{s}{ }^{0} \xi_{(k)(j)}}{2}\left(\frac{{ }^{t} w_{(j)}-{ }^{t} w_{(k)}}{{ }^{0} \xi_{(k)(j)}}-\frac{{ }^{t} \hat{\theta}_{(k)}+{ }^{t} \hat{\theta}_{(j)}}{2}\right) \frac{\cos \left({ }^{t} \theta_{x(k)}\right)+\cos \left({ }^{t} \theta_{x(j)}\right)}{2}
\end{array}\right] \sin \varphi
\end{aligned}
$$




$$
\begin{aligned}
& { }_{0}^{t} \bar{f}_{(k)(j)}^{\theta_{y}}={ }_{0}^{t} \bar{t}_{(k)(j)}^{\theta_{y}}-{ }_{0}^{t} \bar{t}_{(j)(k)}^{\theta_{y}} \\
& =-\left[\begin{array}{l}
\frac{2 a_{b} d_{b}}{{ }^{0} \xi_{(k)(j)}}\left({ }_{0}^{t} \vartheta_{b(k)}+{ }_{0}^{t} \vartheta_{b(j)}\right)+4 b_{b 0}{ }_{0}^{t} s_{b(k)(j)} \\
+\frac{C_{s}{ }^{0} \xi_{(k)(j)}}{2}\left(\frac{{ }^{t} w_{(j)}-{ }^{t} w_{(k)}}{{ }^{0} \xi_{(k)(j)}}-\frac{{ }^{t} \hat{\theta}_{(k)}+{ }^{t} \hat{\theta}_{(j)}}{2}\right) \frac{\cos \left({ }^{t} \theta_{y(k)}\right)+\cos \left({ }^{t} \theta_{y(j)}\right)}{2}
\end{array}\right] \cos \varphi
\end{aligned}
$$

To decide the state of interaction as described in Eq. (53), two common criteria based on the critical bond stretch $[7,8]$ or the critical energy release rate $[16,18,65]$ are used. In this study, the damage criterion based on the critical energy release rate is used. This criterion can be described as [14, 16, 18, 27]

${ }_{0}^{t} \bar{g}_{(k)(j)}<g_{c} \rightarrow$ interaction exists: $\mu_{(k)(j)}=1$

${ }_{0}^{t} \bar{g}_{(k)(j)} \geq g_{c} \rightarrow$ interaction is broken: $\mu_{(k)(j)}=0$

where ${ }_{0}^{t} \bar{g}_{(k)(j)}$ represents the average energy release rate for the interaction between material points $k$ and $j$ at time $t$. The parameter $g_{c}$ represents the average critical energy release rate for one interaction $[16,18]$.

The average energy release rate, ${ }_{0}^{t} \bar{g}_{(k)(j)}$ of the interaction between material points $k$ and $j$ can be calculated as

${ }_{0}^{t} \bar{g}_{(k)(j)}=\frac{1}{2}\left({ }_{0}^{t} g_{(k)(j)}+{ }_{0}^{t} g_{(j)(k)}\right)$

with

$$
\begin{aligned}
& { }_{0}^{t} g_{(k)(j)}=\frac{1}{(\Delta x) h}{ }_{0}^{t} \omega_{(k)(j)}{ }^{0} V_{(k)}{ }^{0} V_{(j)} \\
& { }_{0}^{t} g_{(j)(k)}=\frac{1}{(\Delta x) h}{ }_{0}^{t} \omega_{(j)(k)}{ }^{0} V_{(j)}{ }^{0} V_{(k)}
\end{aligned}
$$

where $\Delta x$ represents the mesh size in the PD discretized model. The terms ${ }_{0}^{t} \omega_{(k)(j)}$ and ${ }_{0}^{t} \omega_{(j)(k)}$ represent micropotentials of the interaction between material point $k$ and $j$. These micropotentials can be calculated as $[27,66]$

$$
\begin{aligned}
& { }_{0}^{t} \omega_{(k)(j)}={ }_{0}^{t} \omega_{(k)(j)}^{u}+{ }_{0}^{t} \omega_{(k)(j)}^{v}+{ }_{0}^{t} \omega_{(k)(j)}^{w}+{ }_{0}^{t} \omega_{(k)(j)}^{\theta_{x}}+{ }_{0}^{t} \omega_{(k)(j)}^{\theta_{y}} \\
& { }_{0}^{t} \omega_{(j)(k)}={ }_{0}^{t} \omega_{(j)(k)}^{u}+{ }_{0}^{t} \omega_{(j)(k)}^{v}+{ }_{0}^{t} \omega_{(j)(k)}^{w}+{ }_{0}^{t} \omega_{(j)(k)}^{\theta_{x}}+{ }_{0}^{t} \omega_{(j)(k)}^{\theta_{y}}
\end{aligned}
$$

where ${ }_{0}^{t} \omega_{(k)(j)}^{u}, \quad{ }_{0}^{t} \omega_{(k)(j)}^{v},{ }_{0}^{t} \omega_{(k)(j)}^{w}, \quad \omega_{(k)(j)}^{\theta_{x}},{ }_{0}^{t} \omega_{(k)(j)}^{\theta_{y}}$ represent the micropotentials at time $t$ corresponding to the force densities ${ }_{0}^{t} \bar{t}_{(k)(j)}^{u},{ }_{0}^{t} \bar{t}_{(k)(j)}^{v}, \quad{ }_{0}^{t} \bar{t}_{(k)(j)}^{w},{ }_{0}^{t} \bar{t}_{(k)(j)}^{\theta_{x}}, \quad{ }_{0}^{t} \bar{t}_{(k)(j)}^{\theta_{y}}$, respectively. Similarly, ${ }_{0}^{t} \omega_{(j)(k)}^{u},{ }_{0}^{t} \omega_{(j)(k)}^{v},{ }_{0}^{t} \omega_{(j)(k)}^{w},{ }_{0}^{t} \omega_{(j)(k)}^{\theta_{x}},{ }_{0}^{t} \omega_{(j)(k)}^{\theta_{y}}$ represent the micropotentials at time $t$ corresponding to the force densities ${ }_{0}^{t} \bar{t}_{(j)(k)}^{u},{ }_{0}^{t} \bar{t}_{(j)(k)}^{v},{ }_{0}^{t} \bar{t}_{(j)(k)}^{w},{ }_{0}^{t} \bar{t}_{(j)(k)}^{\theta_{x}},{ }_{0}^{t} \bar{t}_{(j)(k)}^{\theta_{y}}$, respectively. These micropotentials can be calculated as 


$$
\begin{aligned}
& { }_{0}^{t} \omega_{(k)(j)}^{u}=\frac{1}{h} \int_{0}^{\left.\left.{ }^{t} u_{(j)}\right)^{t} u_{(k)}\right)}{ }_{0}^{t} \bar{t}_{(k)(j)}^{u} d\left(u_{(j)}-u_{(k)}\right) \\
& { }_{0}^{t} \omega_{(k)(j)}^{v}=\frac{1}{h} \int_{0}^{\left.\left({ }^{t} v_{(j)}\right)^{t} v_{(k)}\right)}{ }_{0}^{t} \bar{t}_{(k)(j)}^{v} d\left(v_{(j)}-v_{(k)}\right) \\
& { }_{0}^{t} \omega_{(k)(j)}^{w}=\frac{1}{h} \int_{0}^{\left({ }^{t} w_{(j)}{ }^{-} w_{(k)}\right)}{ }_{0}^{t} \bar{t}_{(k)(j)}^{w} d\left(w_{(j)}-w_{(k)}\right) \\
& { }_{0}^{t} \omega_{(k)(j)}^{\theta_{x}}=\frac{1}{h} \int_{0}^{\left({ }^{t} \theta_{x(j)}{ }^{t} \theta_{x(k)}\right)}{ }_{0}^{t} \bar{t}_{(k)(j)}^{\theta_{x}} d\left(\theta_{x(j)}-\theta_{x(k)}\right) \\
& { }_{0}^{t} \omega_{(k)(j)}^{\theta_{y}}=\frac{1}{h} \int_{0}^{\left.{ }^{t} \theta_{y(j)}{ }^{t} \theta_{y(k)}\right)}{ }_{0}^{t} \bar{t}_{(k)(j)}^{\theta_{y}} d\left(\theta_{y(j)}-\theta_{y(k)}\right)
\end{aligned}
$$

and

$$
\begin{aligned}
& { }_{0}^{t} \omega_{(j)(k)}^{u}=\frac{1}{h} \int_{0}^{\left({ }^{t} u_{(k)}{ }^{t} u_{(j)}\right)}{ }_{0}^{t} \bar{t}_{(j)(k)}^{u} d\left(u_{(k)}-u_{(j)}\right) \\
& { }_{0}^{t} \omega_{(j)(k)}^{v}=\frac{1}{h} \int_{0}^{\left({ }^{t} v_{(k)}{ }^{t} v_{(j)}\right)}{ }_{0}^{t} \bar{t}_{(j)(k)}^{v} d\left(v_{(k)}-v_{(j)}\right) \\
& { }_{0}^{t} \omega_{(j)(k)}^{w}=\frac{1}{h} \int_{0}^{\left.{ }^{t} w_{(k)}{ }^{t} w_{(j)}\right)}{ }_{0}^{t} \bar{t}_{(j)(k)}^{w} d\left(w_{(k)}-w_{(j)}\right) \\
& { }_{0}^{t} \omega_{(j)(k)}^{\theta_{x}}=\frac{1}{h} \int_{0}^{\left({ }^{t} \theta_{x(k)}{ }^{t} \theta_{x(j)}\right)}{ }_{0}^{t} \bar{t}_{(j)(k)} d\left(\theta_{x(k)}-\theta_{x(j)}\right) \\
& { }_{0}^{t} \omega_{(j)(k)}^{\theta_{y}}=\frac{1}{h} \int_{0}^{\left.{ }^{t} \theta_{y(k)}{ }^{t} \theta_{y(j)}\right)}{ }_{0}^{t} \bar{t}_{(j)(k)} \theta_{y} d\left(\theta_{y(k)}-\theta_{y(j)}\right)
\end{aligned}
$$

where the force densities ${ }_{0}^{t} \bar{t}_{(k)(j)}^{u},{ }_{0}^{t} \bar{t}_{(k)(j)}^{v},{ }_{0}^{t} \bar{t}_{(k)(j)}^{w},{ }_{0}^{t} \bar{t}_{(k)(j)} \theta_{0}{ }_{0}^{t} \bar{t}_{(k)(j)}^{\theta_{y}}$ and ${ }_{0}^{t} \bar{t}_{(j)(k)}^{u},{ }_{0}^{t} \bar{t}_{(j)(k)}^{v},{ }_{0}^{t} \bar{t}_{(j)(k)}^{w},{ }_{0}^{t} \bar{t}_{(j)(k)}^{\theta_{x}}$ , ${ }_{0} \bar{t}_{(j)(k)}^{\theta_{y}}$ are given in Eq. (49) and Eq. (50), respectively.

The critical energy release rate for one interaction, $g_{c}$, given in Eq. (55) can be approximated as [16, 18]

$$
g_{c}=\frac{G_{c}}{N_{c}}
$$

where $G_{c}$ represents the critical energy release rate of the material and $N_{c}$ represents the total number of interactions passing through a unit crack area of $A_{0}=h \Delta x$. For the PD discretized 
model of plates and shells with a horizon size of $\delta=3.015 \Delta x$, the number of interactions is $N_{c}=36[14,27]$.

Note that, the damage parameter $\mu_{(k)(j)}$ is also included in the calculation of the dilatations, ${ }_{0}^{t} \vartheta_{(k)},{ }_{0} \underline{\vartheta}_{(k)}$ and the term, ${ }_{0}^{t} \vartheta_{b(k)},{ }_{0} \underline{\vartheta}_{b(k)}$ by modifying Eqs. (41d-e) and Eqs. (47b-c) as

$$
\begin{aligned}
& { }_{0}^{t} \vartheta_{(k)}=d_{i p} \sum_{j=1}^{N} \mu_{(k)(j)}\left({ }_{0}^{t} s_{i p(k)(j)}\right)^{0} V_{(j)} \\
& { }_{0} \underline{\vartheta}_{(k)}=d_{i p} \sum_{j=1}^{N} \mu_{(k)(j)}\left({ }_{0} \underline{S}_{i p(k)(j)}\right){ }^{0} V_{(j)} \\
& { }_{0}^{t} \vartheta_{b(k)}=d_{b} \sum_{j=1}^{N} \mu_{(k)(j)}\left({ }_{0}^{t} s_{b(k)(j)}\right)^{0} V_{(j)} \\
& { }_{0} \underline{\vartheta}_{b(k)}=d_{b} \sum_{j=1}^{N} \mu_{(k)(j)}\left({ }_{0} \underline{S}_{b(k)(j)}\right)^{0} V_{(j)}
\end{aligned}
$$

where the parameters ${ }_{0}^{t} \vartheta_{(k)},{ }_{0}^{t} \vartheta_{b(k)},{ }_{0}^{t} s_{i p(k)(j)},{ }_{0}^{t} s_{b(k)(j)}$ are represented at time $t$. Meanwhile, ${ }_{0} \underline{\vartheta}_{(k)},{ }_{0} \underline{\vartheta}_{b(k)},{ }_{0} \underline{S}_{i p(k)(j)},{ }_{0} \underline{S}_{b(k)(j)}$ are incremental parameters.

In peridynamics, the damage in a structure can be represented by the damage index, $\phi$, which can be defined as [7]

$$
\phi\left(\mathbf{x}_{(k)}, t\right)=1-\frac{\sum_{j=1}^{N} \mu_{(k)(j)} V_{(j)}}{\sum_{j=1}^{N} V_{(j)}}
$$

\section{Numerical implementation}

In peridynamics, the numerical integration over the horizon of a material point is approximated by considering the entire volumes of its family members. However, as shown in Fig. 8(a), material points, shown in red, are not fully inside the horizon of material point $k$, shown in blue. Therefore, the volume correction factors can be applied for these material points to improve the accuracy of the PD calculations $[8,67]$. Moreover, as shown in Fig. 8(b), material points located far from boundaries of the structure, such as material point $k$, shown in blue, has a full circular horizon with all of its family members. However, material points located near boundaries of the structure, such as material point $i$ shown in red, may have a truncated horizon which leads to the missing of its family members. Therefore, the surface correction factors need to be applied for material points near the boundaries of the structure to improve the PD calculations $[8,16]$. 


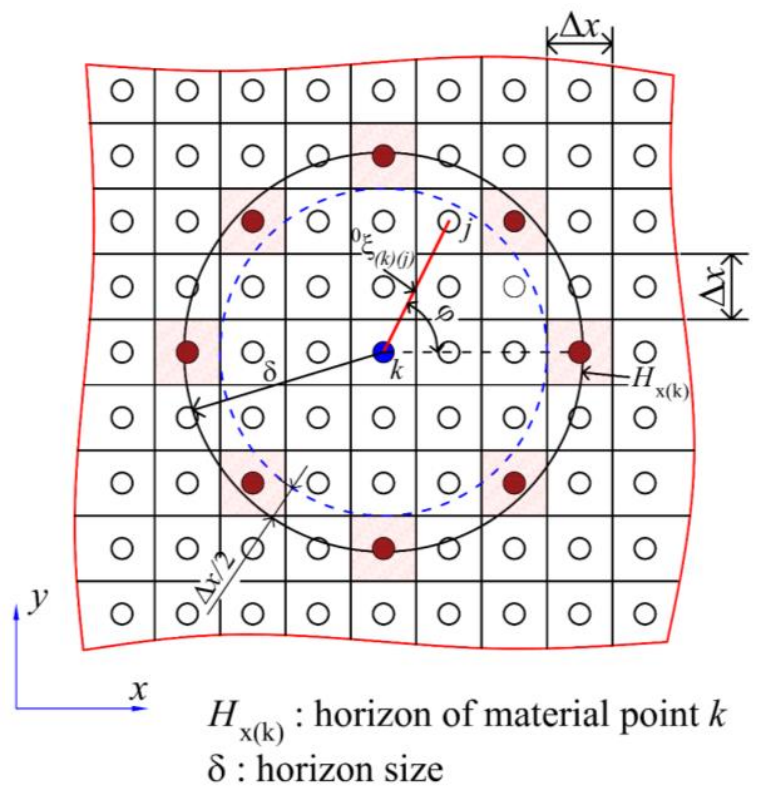

(a)

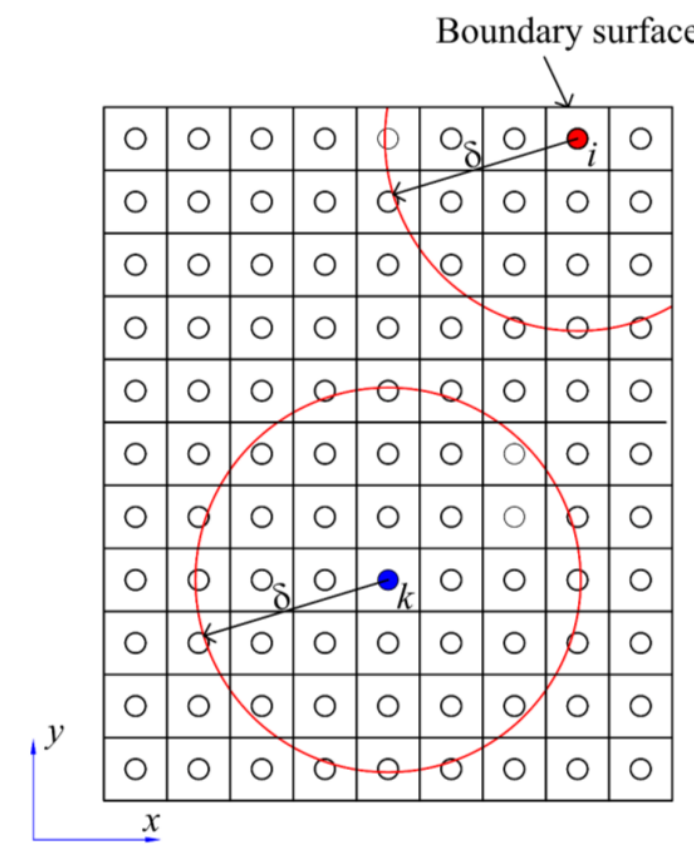

(b)

Fig. 8. Volume and surface corrections (a): volume correction for material points inside the horizon, (b): material points with the truncated horizon and full horizon

As described in Madenci and Oterkus [8], by introducing the volume and surface correction factors, the dilatation terms given in Eq. (41d) and Eq. (47b) can be numerically calculated as

$$
\begin{aligned}
& { }_{0}^{t} \vartheta_{(k)}=d_{i p} \sum_{j=1}^{N}{ }_{0}^{t} s_{i p(k)(j)} v_{(k)(j)} D_{(k)(j)}^{(i p)}{ }^{0} V_{(j)} \\
& { }_{0}^{t} \vartheta_{b(k)}=d_{b} \sum_{j=1}^{N}{ }_{0}^{t} s_{b(k)(j)} v_{(k)(j)} D_{(k)(j)}^{(b)}{ }^{0} V_{(j)}
\end{aligned}
$$

where $v_{(k)(j)}$ represents the volume correction factor for the interaction between material points $k$ and $j$. The term $D_{(k)(j)}^{(i p)}$ and $D_{(k)(j)}^{(b)}$ represent the correction factors for the interaction between material points $k$ and $j$ for dilatation terms of the in-plane and bending deformations, respectively. The calculations of these correction factors are presented in Appendix E.

Similarly, by introducing the volume and surface correction factors, the PD force densities given Eq. (49) and Eq. (50) can be calculated as

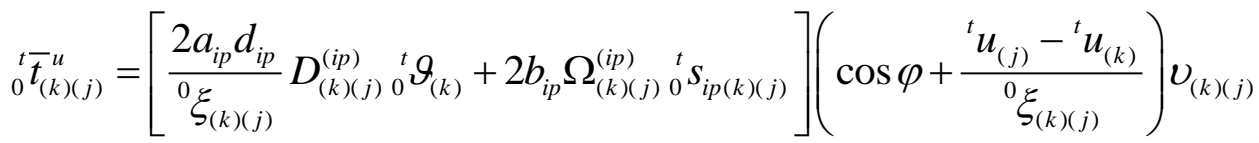

$$
\begin{aligned}
& { }_{0}^{t} \bar{t}_{(k)(j)}^{v}=\left[\frac{2 a_{i p} d_{i p}}{{ }^{0} \xi_{(k)(j)}} D_{(k)(j)}^{(i p)}{ }_{0}^{t} \vartheta_{(k)}+2 b_{i p} \Omega_{(k)(j)}^{(i p)}{ }_{0}^{t} s_{i p(k)(j)}\right]\left(\sin \varphi+\frac{{ }^{t} v_{(j)}-{ }^{t} v_{(k)}}{{ }^{0} \xi_{(k)(j)}}\right) v_{(k)(j)}
\end{aligned}
$$




$$
\begin{aligned}
& { }_{0}^{t} \bar{t}_{(k)(j)}^{w}=\left(\frac{2 a_{i p} d_{i p}}{{ }^{0} \xi_{(k)(j)}} D_{(k)(j) 0}^{(i p)}{ }^{t} \vartheta_{(k)}+2 b_{i p} \Omega_{(k)(j) 0}^{(i p)}{ }^{t} s_{i p(k)(j)}\right) \frac{{ }^{t} w_{(j)}-{ }^{t} w_{(k)}}{{ }^{0} \xi_{(k)(j)}} v_{(k)(j)} \\
& +\frac{1}{2} C_{s}\left(\frac{{ }^{t} w_{(j)}-{ }^{t} w_{(k)}}{{ }^{0} \xi_{(k)(j)}}-\frac{{ }^{t} \hat{\theta}_{(k)}+{ }^{t} \hat{\theta}_{(j)}}{2}\right) \Omega_{(k)(j)}^{(s h)} v_{(k)(j)} \\
& { }_{0}^{t} \bar{t}_{(k)(j)} \theta_{x}=\left[\begin{array}{l}
\frac{2 a_{b} d_{b}}{{ }^{0} \xi_{(k)(j)}} D_{(k)(j)}^{(b)}{ }_{0}^{t} \vartheta_{b(k)}+2 b_{b 0}{ }^{t} s_{b(k)(j)} \Omega_{(k)(j)}^{(b)} \\
+\frac{C_{s}{ }^{0} \xi_{(k)(j)}}{2}\left(\frac{{ }^{t} w_{(j)}-{ }^{t} w_{(k)}}{{ }^{0} \xi_{(k)(j)}}-\frac{{ }^{t} \hat{\theta}_{(k)}+{ }^{t} \hat{\theta}_{(j)}}{2}\right) \frac{\cos \left({ }^{t} \theta_{x(k)}\right)}{2} \Omega_{(k)(j)}^{(s h)}
\end{array}\right] v_{(k)(j)} \sin \varphi \\
& { }_{0}^{t} \bar{t}_{(k)(j)}^{\theta_{y}}=-\left[\begin{array}{l}
\frac{2 a_{b} d_{b}}{{ }^{0} \xi_{(k)(j)}} D_{(k)(j)}^{(b)}{ }_{0}{ }^{t} \vartheta_{b(k)}+2 b_{b}{ }_{0}^{t} s_{b(k)(j)} \Omega_{(k)(j)}^{(b)} \\
+\frac{C_{s}{ }^{0} \xi_{(k)(j)}}{2}\left(\frac{{ }^{t} w_{(j)}-{ }^{t} w_{(k)}}{{ }^{0} \xi_{(k)(j)}}-\frac{\left.{ }^{t} \hat{\theta}_{(k)}+{ }^{t} \hat{\theta}_{(j)}\right)}{2}\right) \frac{\cos \left({ }^{t} \theta_{y(k)}\right)}{2} \Omega_{(k)(j)}^{(s h)}
\end{array}\right] v_{(k)(j)} \cos \varphi
\end{aligned}
$$

and

$$
\begin{aligned}
& { }_{0}^{t} \bar{t}_{(j)(k)}^{u}=-\left[\frac{2 a_{i p} d_{i p}}{{ }^{0} \xi_{(k)(j)}} D_{(k)(j) 0}^{(i p)}{ }_{0}^{t} \vartheta_{(j)}+2 b_{i p} \Omega_{(k)(j)}^{(i p)}{ }_{0}^{t} s_{i p(k)(j)}\right]\left(\cos \varphi+\frac{{ }^{t} u_{(j)}-{ }^{t} u_{(k)}}{{ }^{0} \xi_{(k)(j)}}\right) v_{(k)(j)} \\
& { }_{0}^{t} \bar{t}_{(j)(k)}^{v}=-\left[\frac{2 a_{i p} d_{i p}}{{ }^{0} \xi_{(k)(j)}} D_{(k)(j) 0}^{(i p)}{ }_{0}^{t} \vartheta_{(j)}+2 b_{i p} \Omega_{(k)(j)}^{(i p)}{ }_{0}^{t} s_{i p(k)(j)}\right]\left(\sin \varphi+\frac{{ }^{t} v_{(j)}-{ }^{t} v_{(k)}}{{ }^{0} \xi_{(k)(j)}}\right) v_{(k)(j)} \\
& { }_{0}^{t} \bar{t}_{(j)(k)}^{w}=-\left(\frac{2 a_{i p} d_{i p}}{{ }^{0} \xi_{(k)(j)}} D_{(k)(j)}^{(i p)}{ }_{0}^{t} \vartheta_{(j)}+2 b_{i p} \Omega_{(k)(j)}^{(i p)}{ }_{0}^{t} s_{i p(k)(j)}\right) \frac{{ }^{t} w_{(j)}-{ }^{t} w_{(k)}}{{ }^{0} \xi_{(k)(j)}} v_{(k)(j)} \\
& -\frac{1}{2} C_{s}\left(\frac{{ }^{t} w_{(j)}-{ }^{t} w_{(k)}}{{ }^{0} \xi_{(k)(j)}}-\frac{{ }^{t} \hat{\theta}_{(k)}+{ }^{t} \hat{\theta}_{(j)}}{2}\right) \Omega_{(k)(j)}^{(s h)} v_{(k)(j)} \\
& { }_{0}^{t} \bar{t}_{(j)(k)}^{\theta_{x}}=-\left[\begin{array}{l}
\frac{2 a_{b} d_{b}}{{ }^{0} \xi_{(k)(j)}} D_{(k)(j) 0}^{(b)}{ }_{0}^{t} \vartheta_{b(j)}+2 b_{b} \Omega_{(k)(j) 0}^{(b)}{ }^{t} S_{b(k)(j)} \\
+\frac{C_{s}{ }^{0} \xi_{(k)(j)}}{2}\left(\frac{{ }^{t} w_{(j)}-{ }^{t} w_{(k)}}{{ }^{0} \xi_{(k)(j)}}-\frac{{ }^{t} \hat{\theta}_{(k)}+{ }^{t} \hat{\theta}_{(j)}}{2}\right) \frac{\cos \left({ }^{t} \theta_{x(j)}\right)}{2} \Omega_{(k)(j)}^{(s h)}
\end{array}\right] v_{(k)(j)} \sin \varphi \\
& { }_{0}^{t} \bar{t}_{(j)(k)}^{\theta_{y}}=\left[\begin{array}{l}
\frac{2 a_{b} d_{b}}{{ }^{0} \xi_{(k)(j)}} D_{(k)(j)}^{(b)}{ }_{0}^{t} \vartheta_{b(j)}+2 b_{b} \Omega_{(k)(j) 0}^{(b)}{ }^{t} S_{b(k)(j)} \\
+\frac{C_{s}{ }^{0} \xi_{(k)(j)}}{2}\left(\frac{{ }^{t} w_{(j)}-{ }^{t} w_{(k)}}{{ }^{0} \xi}-\frac{{ }^{t} \hat{\theta}_{(k)}+{ }^{t} \hat{\theta}_{(j)}}{2}\right) \frac{\cos \left({ }^{t} \theta_{y(j)}\right)}{2} \Omega_{(k)(j)}^{(s h)}
\end{array}\right] v_{(k)(j)} \cos \varphi
\end{aligned}
$$

where $\Omega_{(k)(j)}^{(i p)}, \Omega_{(k)(j)}^{(s h)}$, and $\Omega_{(k)(j)}^{(b)}$ represent the surface correction factors of the interaction between material points $k$ and $j$ for the in-plane, shear, and bending deformations, respectively. The calculations of these correction factors are presented in Appendix E. 
In peridynamics, the equations of motion given in Eqs. (54a-b) can be solved by using the meshless scheme [7]. The PD discretized model is represented by material points. For dynamic problems, the equations of motion given in Eqs. (54a-b) can be solved by using the time explicit integration scheme [8]. For static and quasi-static problems, the adaptive dynamic relaxation (ADR) method can be used [27, 68, 69]. Details of the adaptive dynamic relaxation method for nonlinear PD analysis of plate are presented in Appendix D. The numerical procedure for PD nonlinear analysis and damage prediction of plates is shown in Fig. 9.

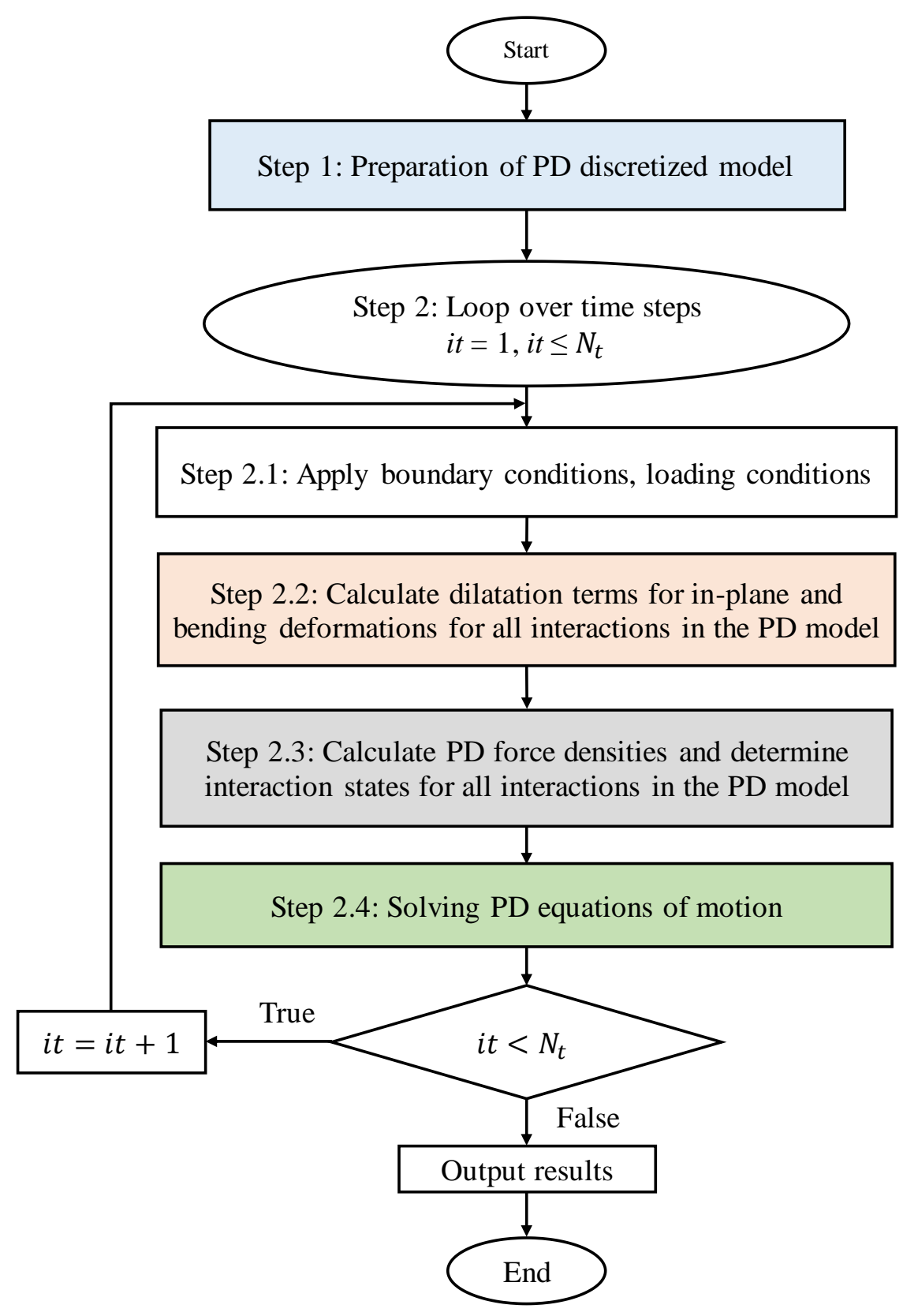

Fig. 9. Numerical procedure for PD nonlinear analysis of plates

As shown in Fig. 9, the numerical procedure for PD nonlinear analysis of plates includes two main steps. In step 1, the PD discretized model is prepared as shown in Fig. 10. In step 2, at each time step, first, boundary and loading conditions are applied in step 2.1. Next, the dilatation terms for in-plane and bending deformations of material points are calculated in step 2.2. The PD force densities are calculated, and the states of interactions are determined in Step 2.3. Finally, the PD equations of motions for all material points in the discretized model are 
solved in step 2.4. Details of the procedures in step 2.2, 2.3, and 2.4 are presented in Fig. 11, Fig. 12, and Fig. 13, respectively.

Step 1: Preparation of PD discretized model

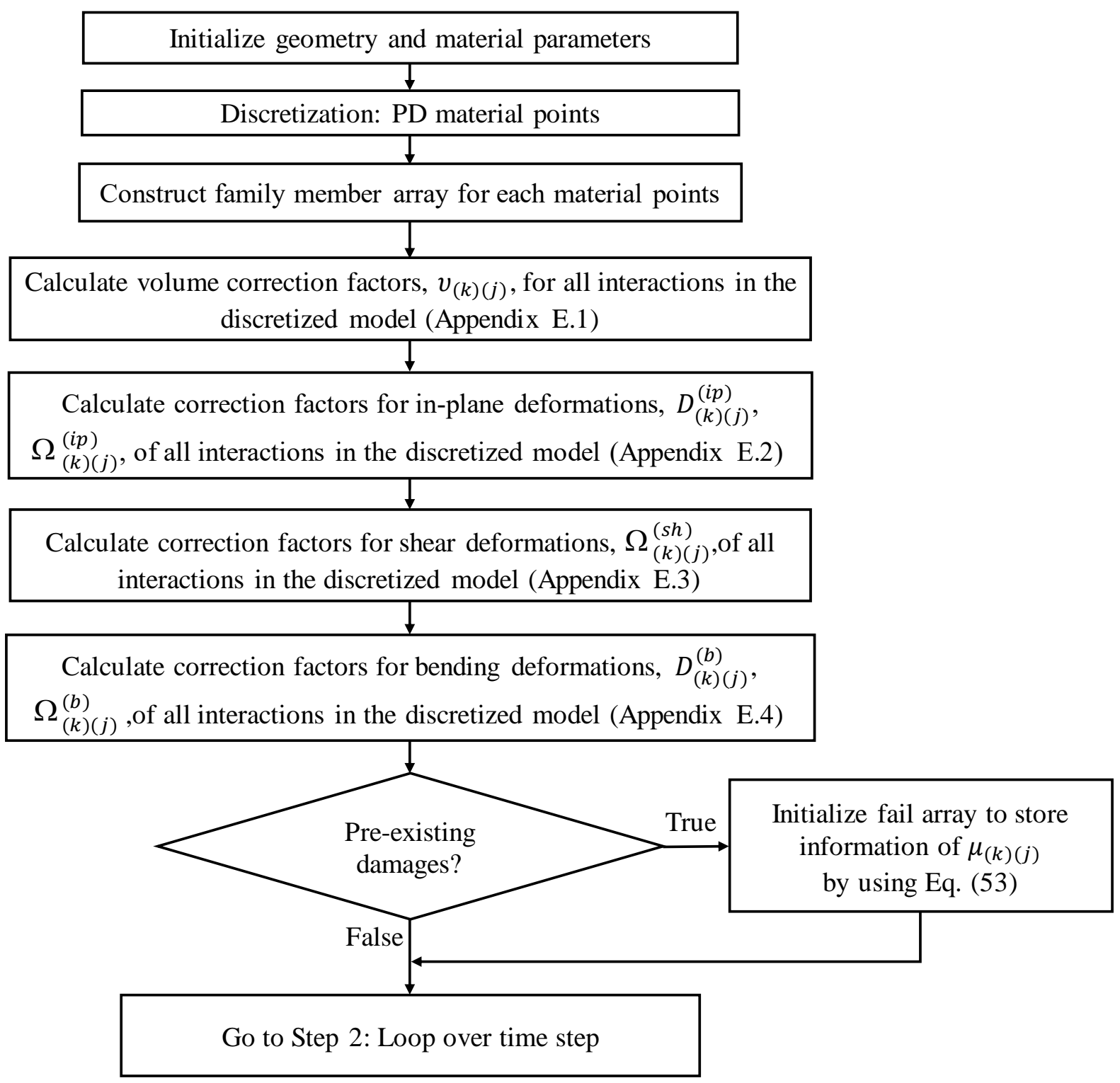

Fig. 10. Step 1: preparation of the PD discretized model 
Step 2.2: Calculate dilatation terms for in-plane and bending deformations for all interactions in the PD model

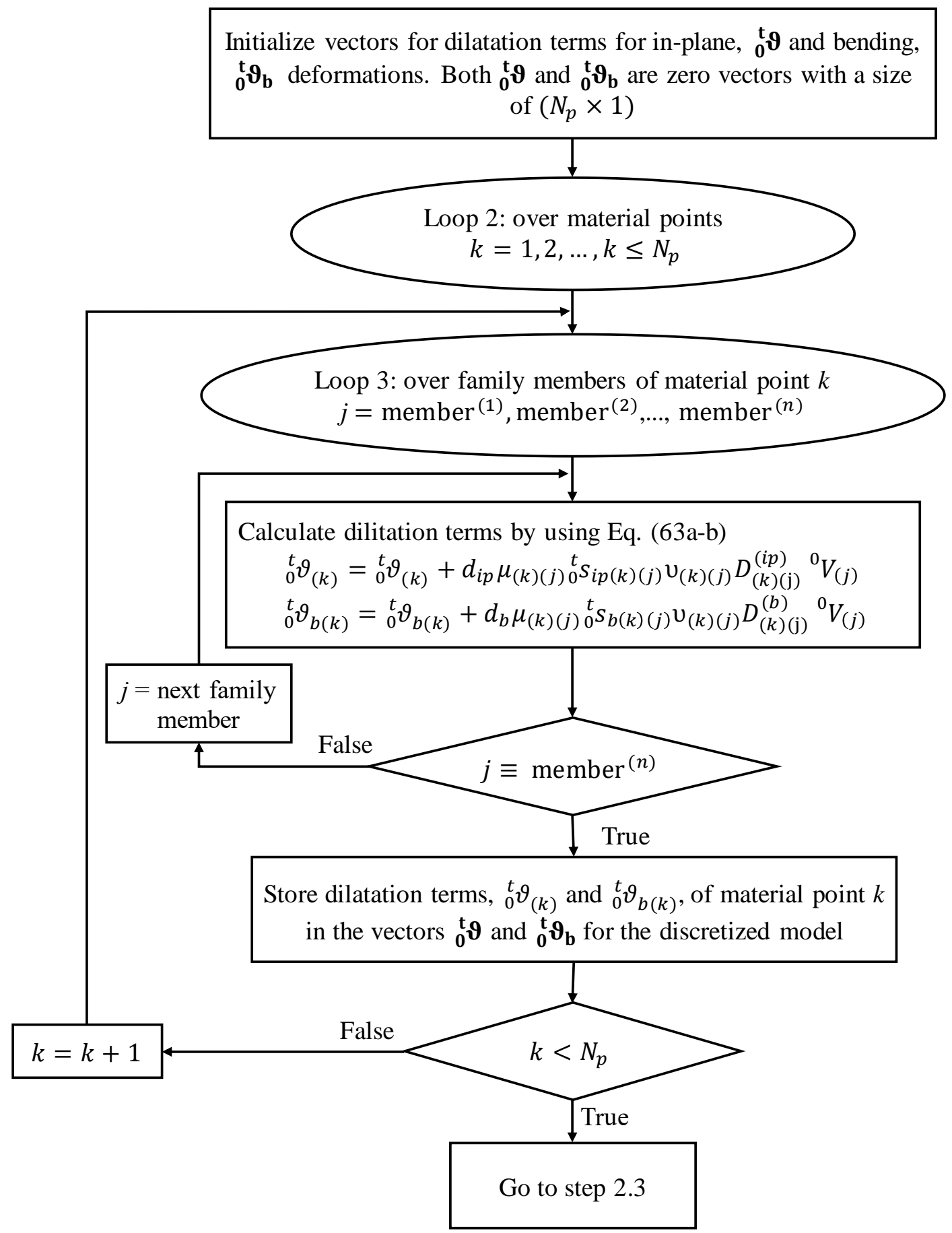

Fig. 11. Step 2.2: Calculate dilatation terms for in-plane and bending deformations of all material points in the PD discretized model ( $N_{P}$ represents number of material points in the discretized model) 


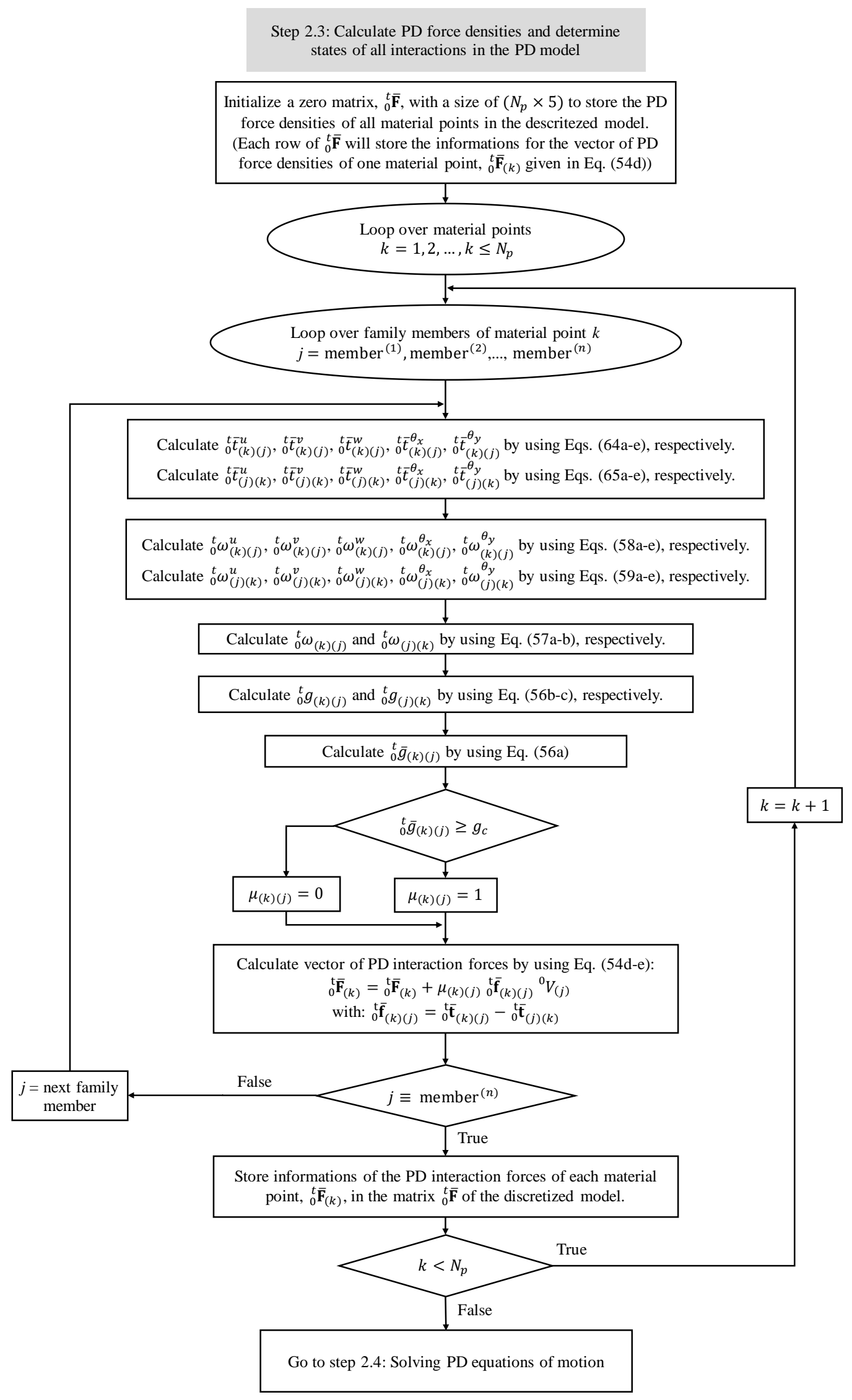

Fig. 12. Step 2.3: Calculate PD force densities and determine states of interactions in the PD discretized model 


\section{Step 2.4: Solving PD equations of motion}
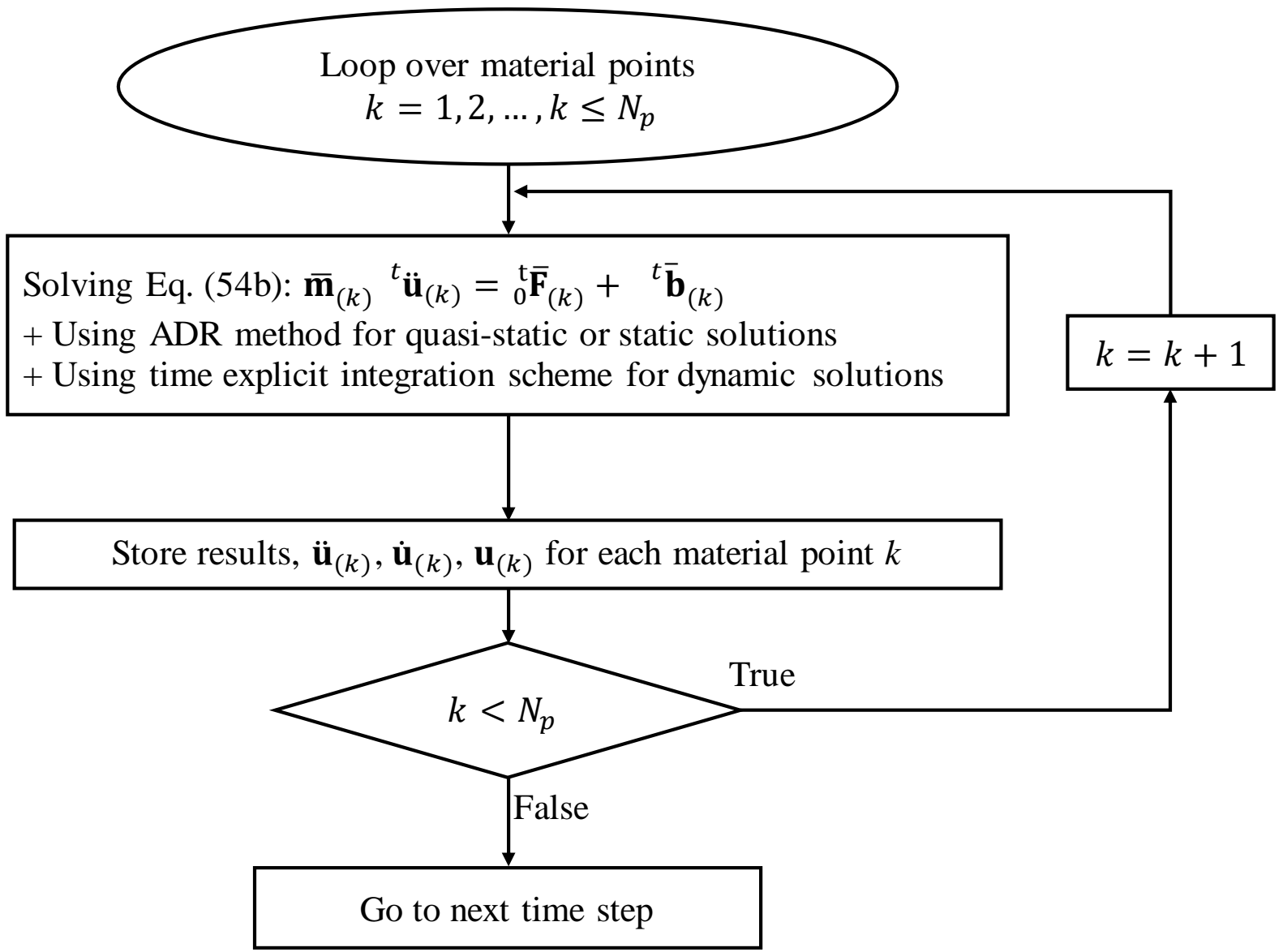

Fig. 13. Step 2.4: solving PD equations of motion

\section{Numerical results}

In this section, large deformations of plates are predicted by using the proposed nonlinear PD model. First, the verifications for the nonlinear model are presented in Sections 7.1 and 7.2. The nonlinear PD results are compared with the nonlinear FEA results. Next, in Section 7.3, 7.4, and 7.5, the nonlinear PD model is used to predict progressive damages on plates subjected to different loading conditions. For static and quasi-static loading conditions, the adaptive dynamic relaxation method $[68,69]$ is used. Details of the adaptive dynamic relaxation method for nonlinear PD analysis of plate are presented in Appendix D. All the nonlinear PD simulations are implemented in MATLAB 2018b.

\subsection{A plate subjected to bending}

In this section, a square plate subjected to bending as shown in Fig. 14 is investigated. The plate has dimensions of $L=W=1 \mathrm{~m}$ and the thickness of $h=L / 10$. The plate is fixed on the left edge and it is subjected to bending, $m_{y}=3 \times 10^{7} \mathrm{Nm} / \mathrm{m}$ on the right edge. The plate is made of steel with the elastic modulus of $E=2 \times 10^{11} \mathrm{~N} / \mathrm{m}^{2}$ and Poisson's ratio of $v=0.3$. 
In the PD model, the plate is uniformly discretized with a mesh size of $\Delta x=L / 100$. To apply boundary conditions, three fictitious layers of material points are added on the left side of the plate as shown in Fig. 14(b). All DOFs of the fictitious points as well as the DOFs of the material points located at $x=0$ are set equal to zero. To apply the loading condition, the bending per unit area, $\bar{m}_{y}=m_{y} / \Delta x$ is applied to the material points located at $x=L$. In the FEA by using ANSYS, the SHELL181 element with the same mesh size $\Delta x=L / 100$ is used.

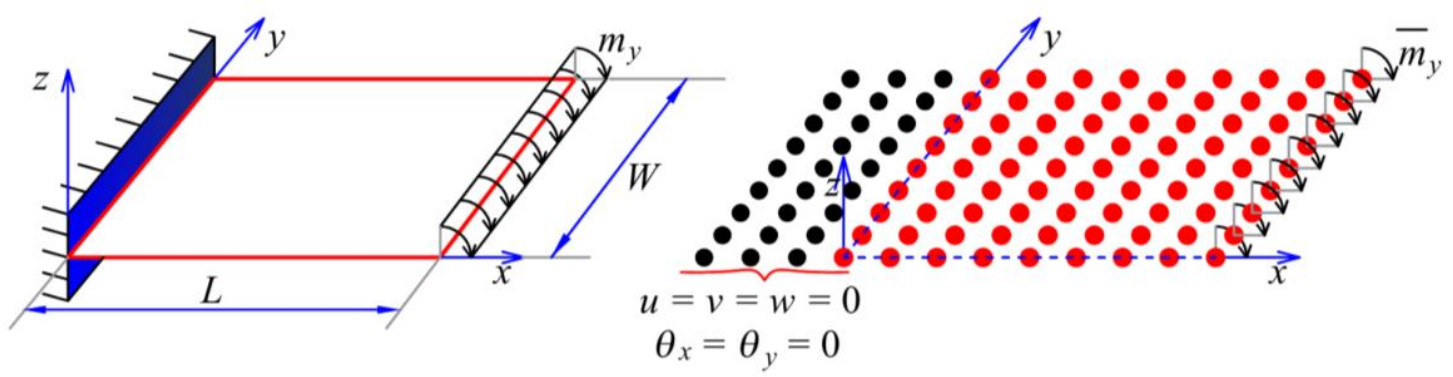

(a)

(b)

Fig. 14. A square plate subjected to bending (a): geometry, (b): PD discretized model

To decide a suitable horizon size for the PD nonlinear model, the deformation of the plate is predicted by using the nonlinear PD model with different horizon sizes. The nonlinear PD results for significant DOFs, $\left(u, w\right.$, and $\left.\theta_{y}\right)$, of the material points located at $(x=L, y=W / 2)$ and $(x=3 L / 4, y=W / 4)$ are compared with the nonlinear FEA results of the nodes located at the same locations as shown in Fig. 15. The relative errors between the nonlinear PD and nonlinear FEA results are calculated as

$\operatorname{Error}(q)=\frac{\left|q^{P D}-q^{F E A}\right|}{\left|q^{F E A}\right|} \times 100(\%)$

where $q$ represents the degree of freedom, $q^{P D}$ and $q^{F E A}$ represent the nonlinear PD and nonlinear FEA results for the degree of freedom $q$, respectively.

As can be observed from Fig. 15, the nonlinear PD results start to converge to the nonlinear FEA results when the horizon size $\delta \geq 3 \Delta x$. The relative errors between the nonlinear PD and nonlinear FEA results for the material point located at $(x=3 L / 4, y=W / 4)$ are smaller than $2 \%$. Meanwhile, these relative errors for the material point located at $(x=L, y=W / 2)$ are smaller than $5 \%$. Therefore, to minimize the computational cost in the PD simulation, the horizon size $\delta \geq 3.015 \Delta x$ is chosen. 


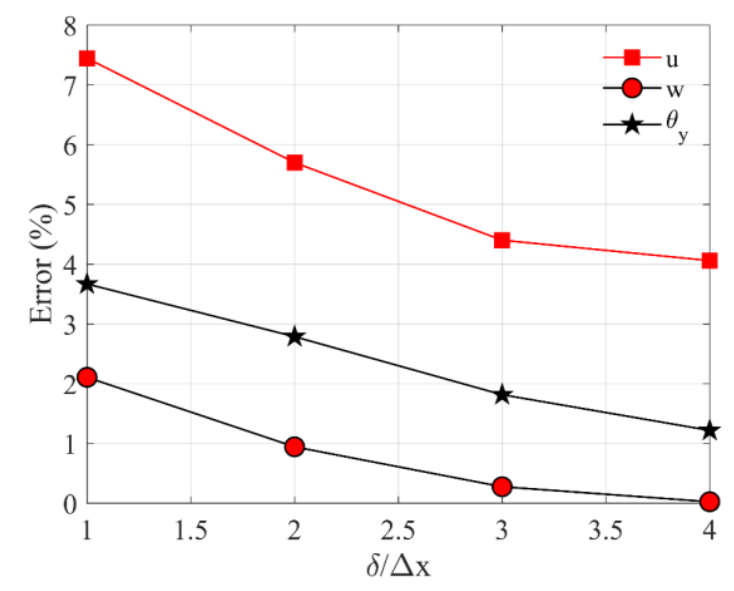

(a)

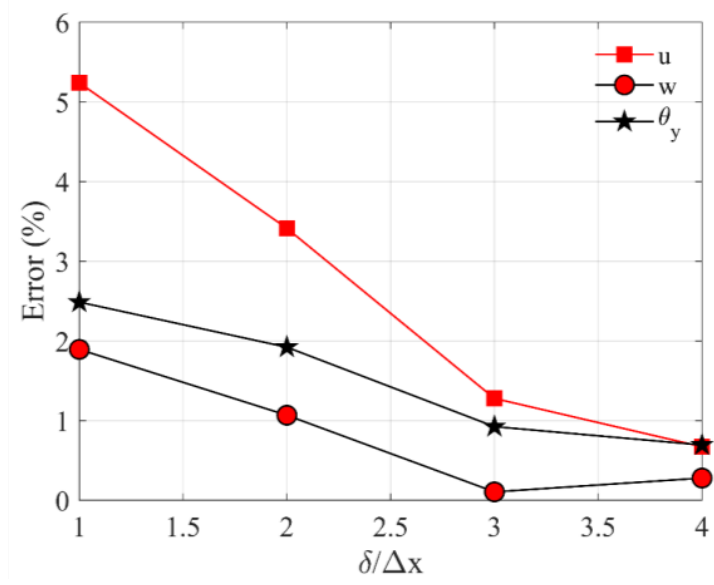

(b)

Fig. 15. Relative errors between the nonlinear FEA and nonlinear PD results with different horizon sizes for significant DOFs: $u, w$, and $\theta_{y}$ of material points located at (a):

$$
(x=L, y=W / 2),(\mathrm{b}):(x=3 L / 4, y=W / 4)
$$

Fig. 16-Fig. 20 show the comparison of nonlinear results for all DOFs in the deformed configuration of the plate. As can be seen from the figures, the nonlinear PD results and nonlinear FEA results have good agreements.

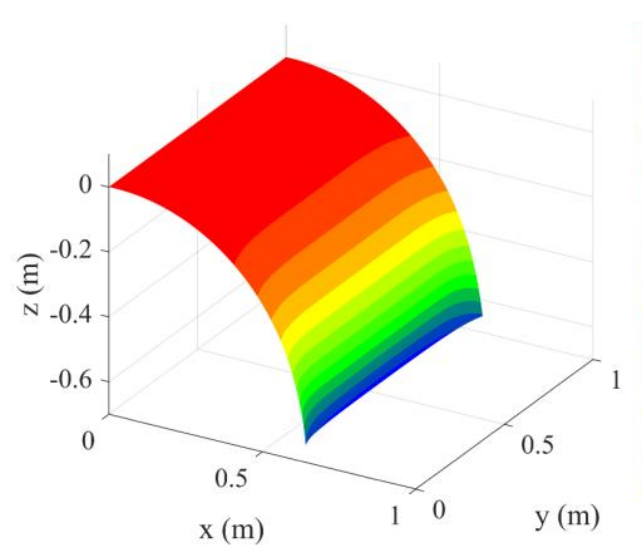

(a)
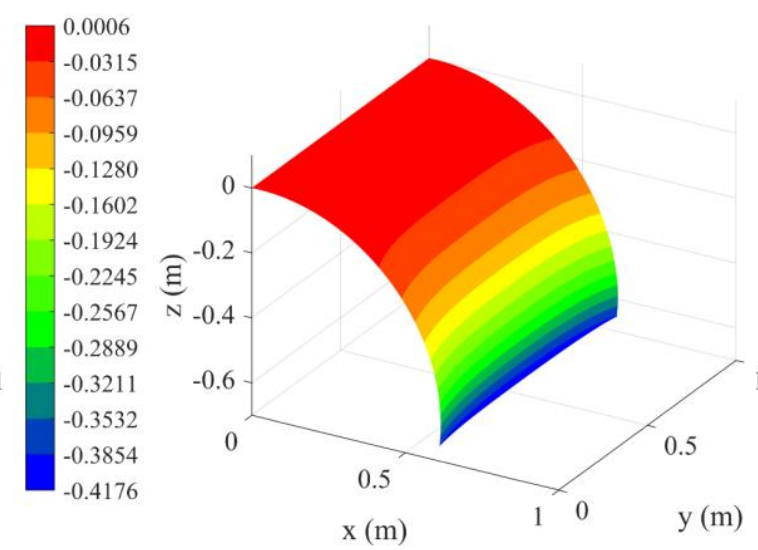

(b)

Fig. 16. Variation of displacement $u(\mathrm{~m})$ in (a): nonlinear PD, (b): nonlinear FEA 


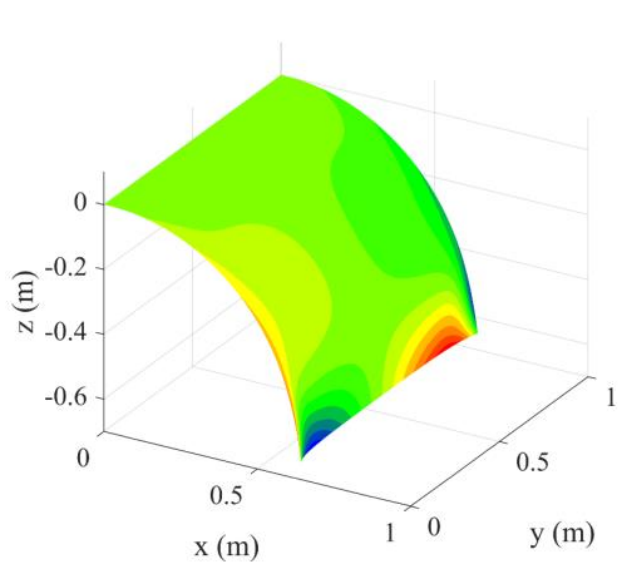

(a)

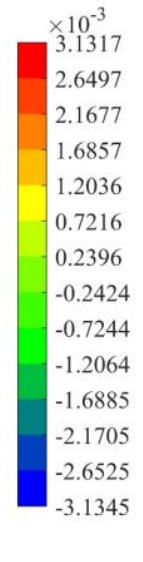

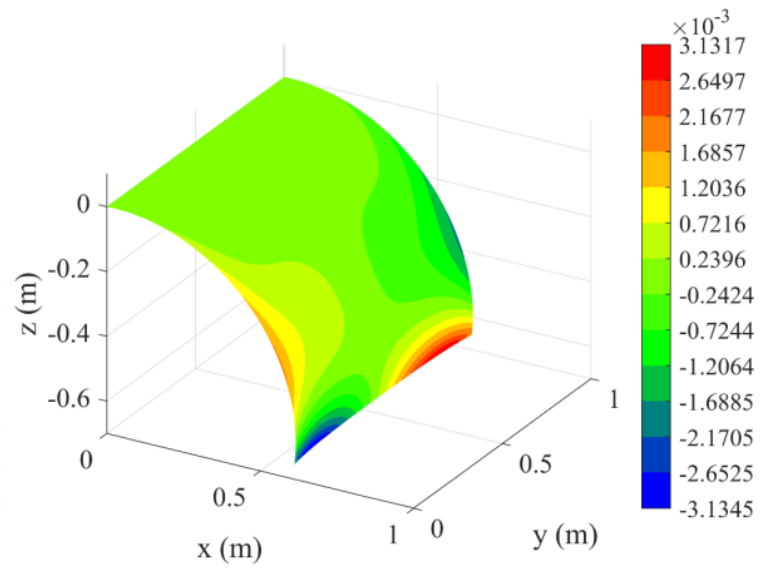

(b)

Fig. 17. Variation of displacement $v(\mathrm{~m})$ in (a): nonlinear PD, (b): nonlinear FEA

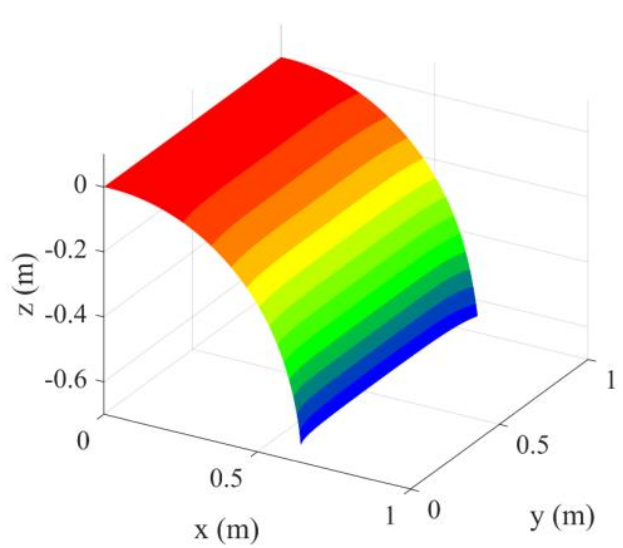

(a)

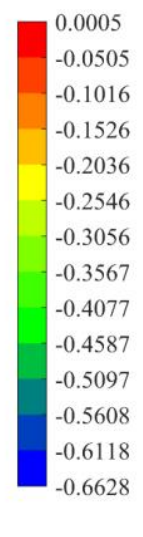

$-0.6118$

$$
\left(\frac{100}{10}\right.
$$

$w(m)$

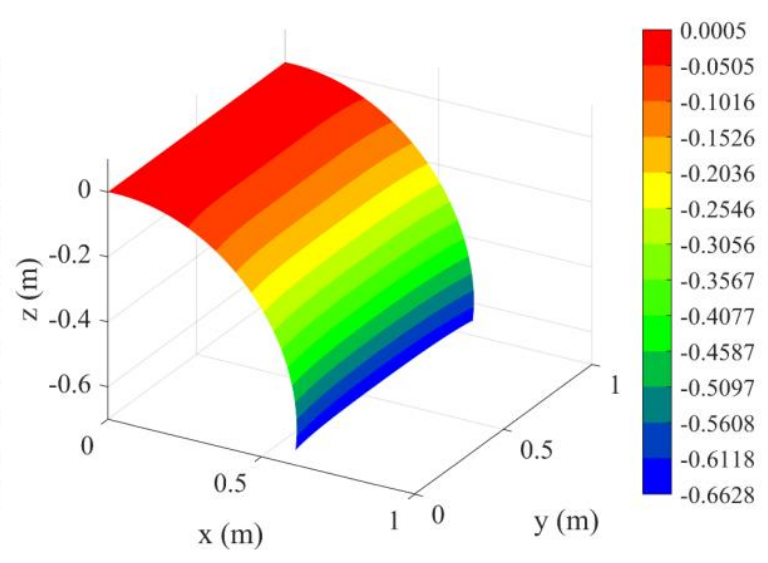

(b)

Fig. 18. Variation of displacement $w(\mathrm{~m})$ in (a): nonlinear PD, (b): nonlinear FEA

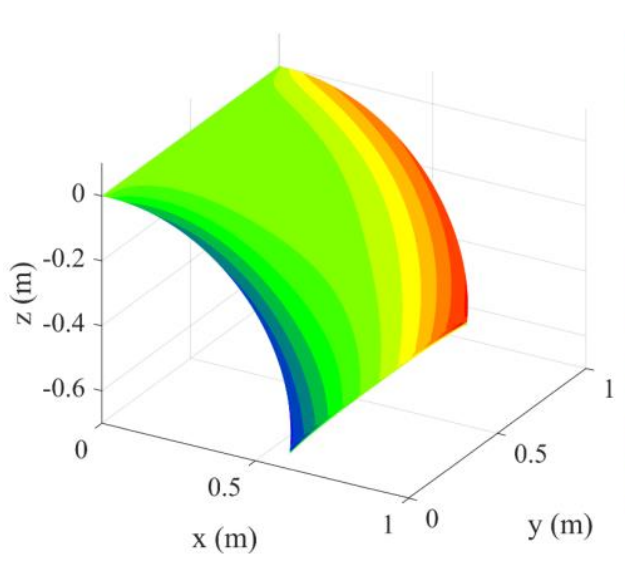

(a)

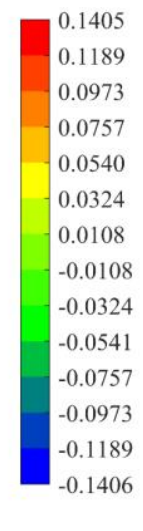

1406

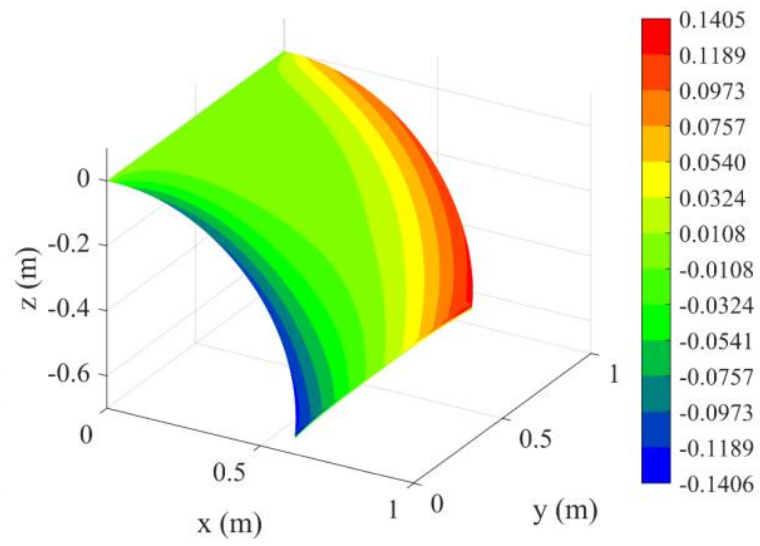

(b)

Fig. 19. Variation of rotation $\theta_{x}(\mathrm{rad})$ in (a): nonlinear PD, (b): nonlinear FEA 


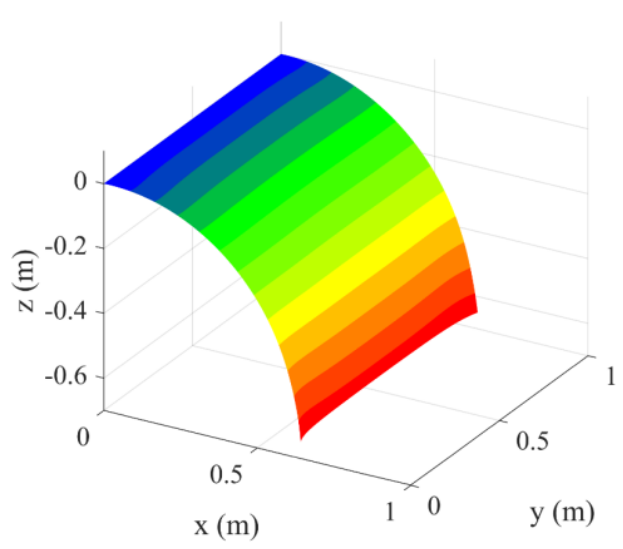

(a)

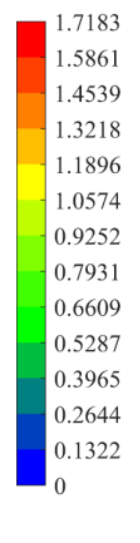

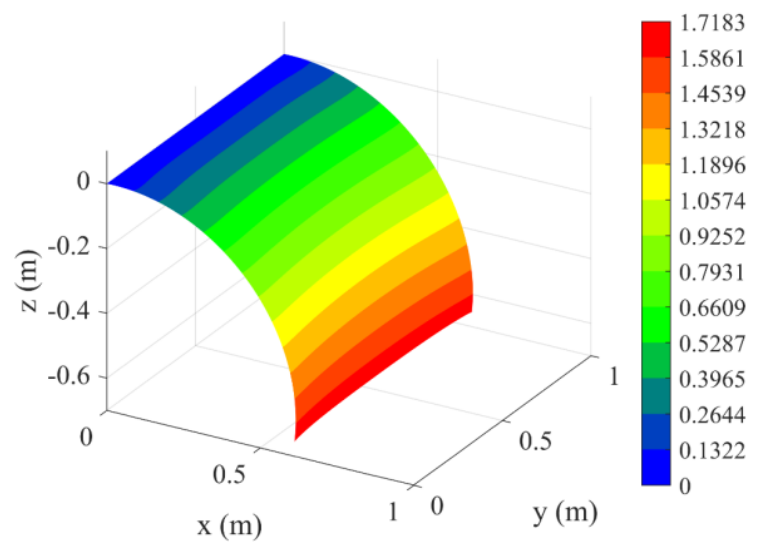

(b)

Fig. 20. Variation of rotation $\theta_{y}(\mathrm{rad})$ in (a): nonlinear PD, (b): nonlinear FEA

Fig. 21 shows the comparison of the deformed configuration of the centreline at $y=W / 2$ predicted by nonlinear PD analysis and nonlinear FEA. As it can be observed from the figure, the deformed configurations captured by both methods have a very good match except a small difference at the right end of the plate.

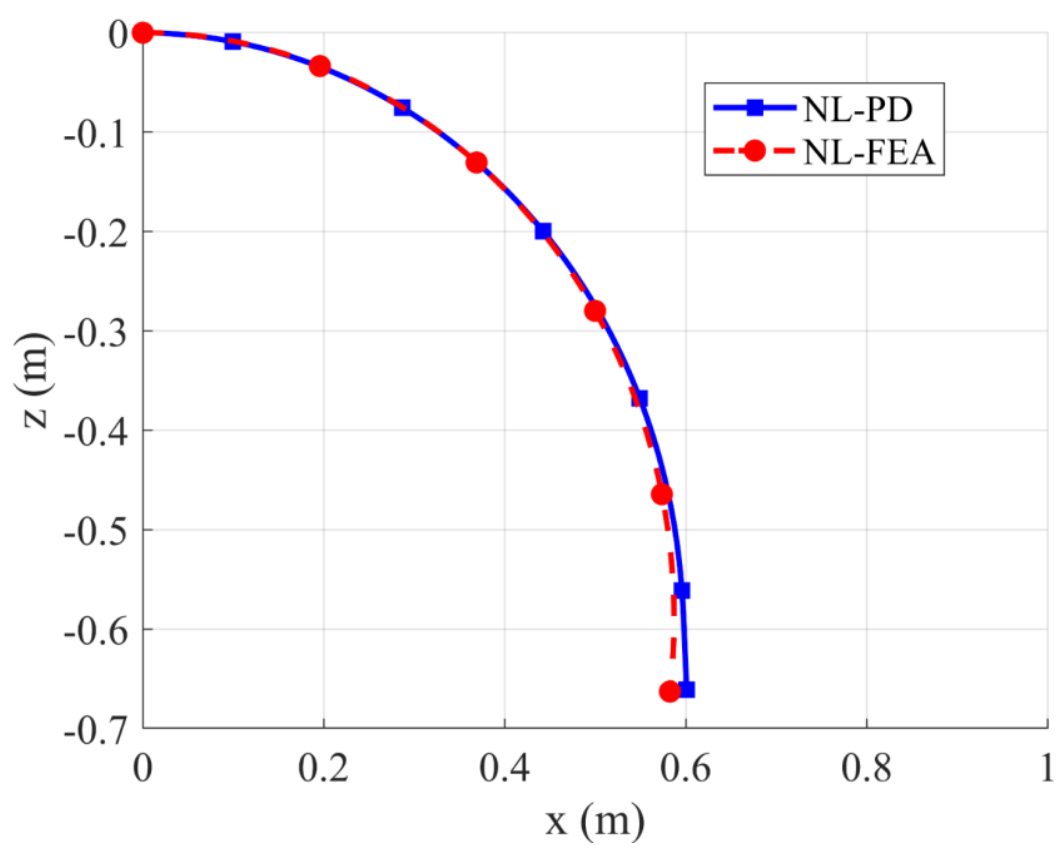

Fig. 21. Deformed configurations of the centreline $y=W / 2$

\subsection{A plate subjected to vertical shear forces}

In this section, a rectangular plate with the dimensions of $L=1 \mathrm{~m}, W=0.2 \mathrm{~m}$, and thickness of $h=L / 50$ is investigated as shown in Fig. 22. The plate is fixed on the left edge and it is subjected to vertical shear forces on the right edge. The values of the distributed forces are defined as $f_{z}=\mathrm{n} \times 10^{4} \mathrm{~N} / \mathrm{m}$ with $n=5,10,50,100$. The plate has the elastic modulus of $E=2 \times 10^{11} \mathrm{~N} / \mathrm{m}^{2}$ and Poisson's ratio of $v=0.4$. 
In the PD model, the plate is uniformly discretized with a mesh size of $\Delta x=L / 100$. The same method discussed in the previous section is used for applying boundary conditions. To apply the loading condition, the force per unit area $\bar{b}_{z}=f_{z} / \Delta x$ is applied to the material points located at $x=L$. In the nonlinear FEA, the SHELL181 element and the same mesh size are used.

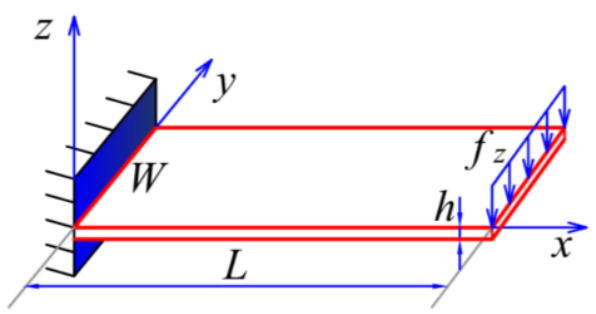

(a)

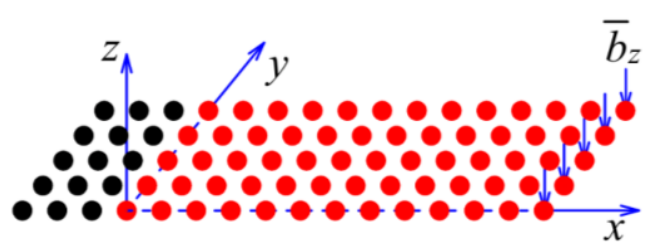

(b)

Fig. 22. A plate subjected to vertical shear forces (a): geometry, (b): PD discretized model

Fig. 23 shows the deformed configurations of the plate in different loading conditions captured by the nonlinear PD model. Fig. 24 shows the deformed configurations of the plate along the centreline $y=W / 2$. As can be seen from Fig. 24, the nonlinear PD and nonlinear FEA results show good agreements for all loading conditions. The difference between the two results for the large loading condition with $n=100$ is still small which can verify the accuracy of the nonlinear PD model.

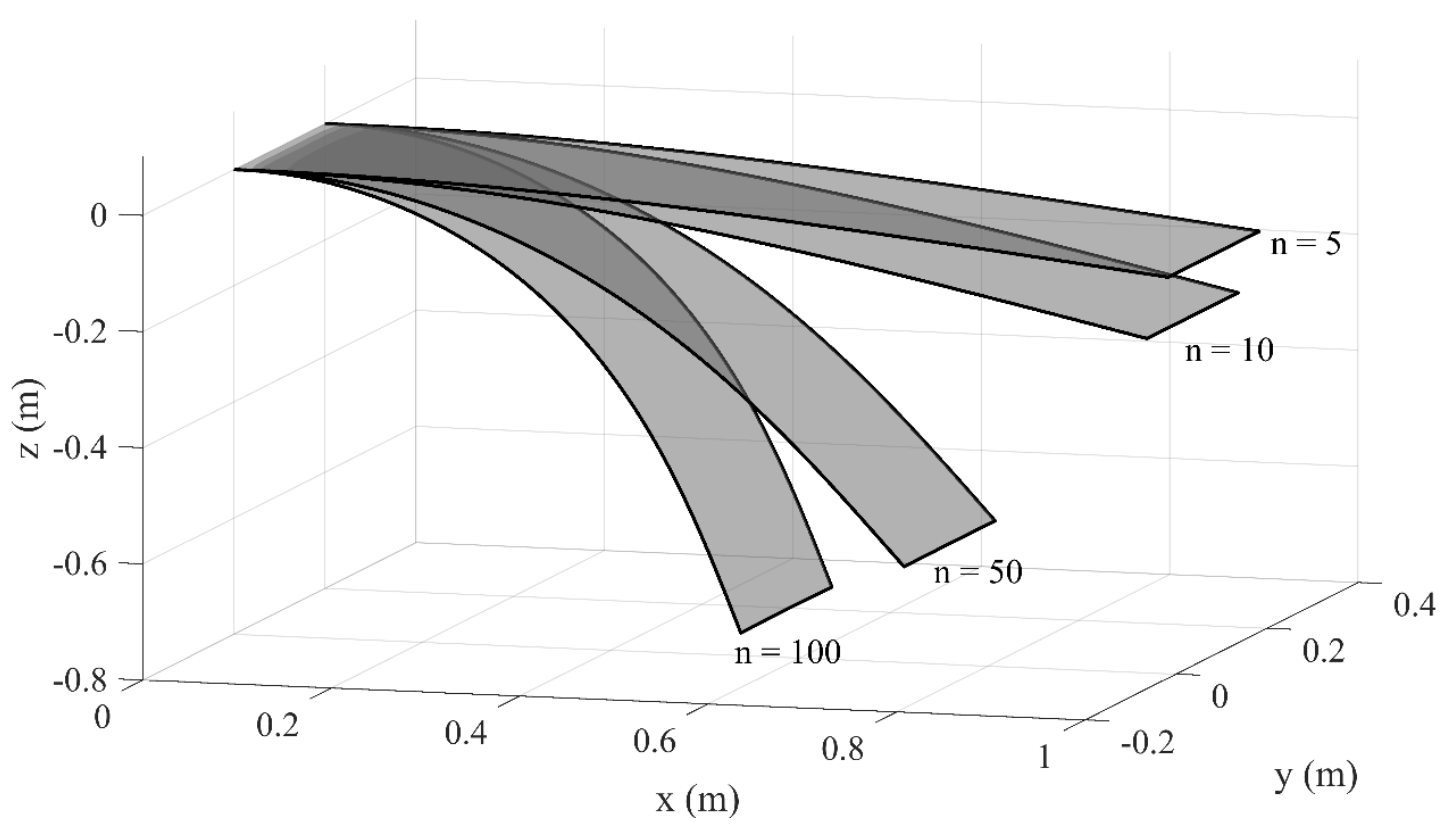

Fig. 23. Deformed configurations of the plate subjected to distributed force $f_{z}=\mathrm{n} \times 10^{4} \mathrm{~N} / \mathrm{m}$ with $n=5,10,50,100$ captured by the nonlinear PD model 


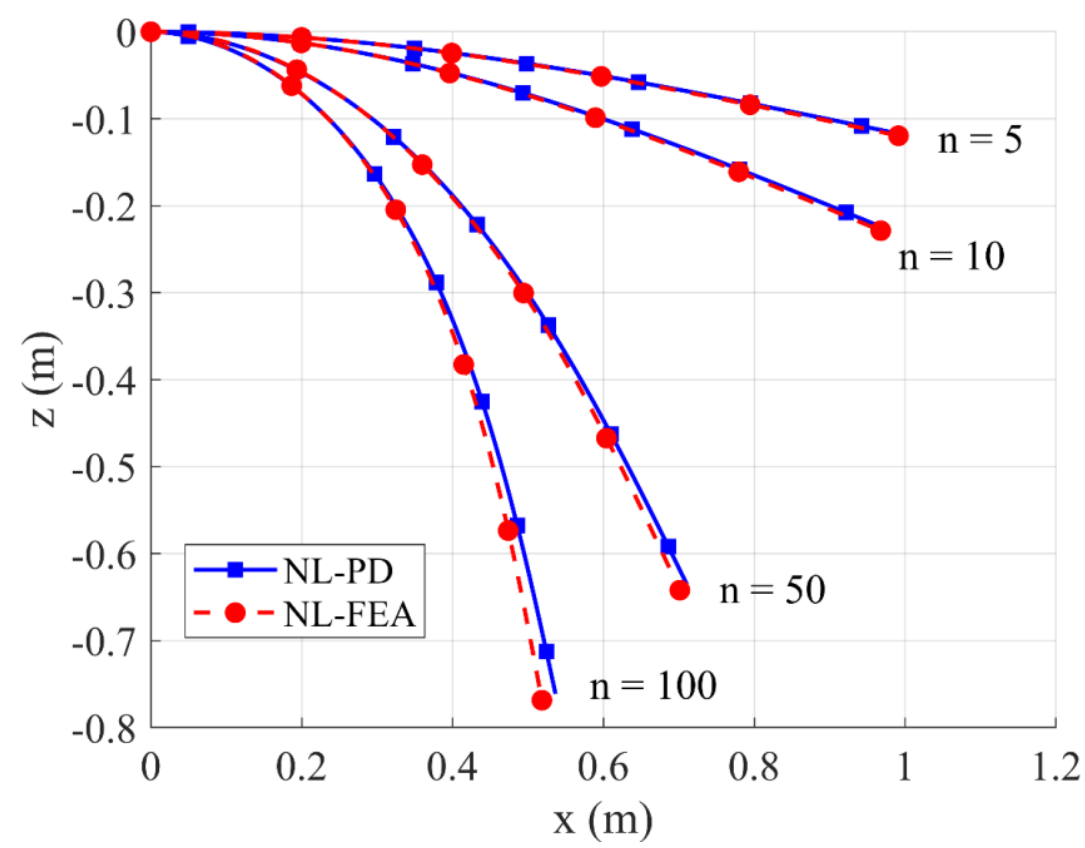

Fig. 24. Deformed configurations of the centreline at $y=W / 2$ of the plate subjected to distributed force $f_{z}=\mathrm{n} \times 10^{4} \mathrm{~N} / \mathrm{m}$ with $n=5,10,50,100$

\subsection{Out-of-plane stretching and tearing of a plate}

After verifying the accuracy of the nonlinear PD model, in this section, progressive damage on a square plate with pre-existing crack subjected to out-of-plane stretching and tearing is investigated. The experimental details for this problem can be found in [70]. The square plate has dimensions of $L=W=0.203 \mathrm{~m}$ and thickness of $h=8 \times 10^{-4} \mathrm{~m}$. The plate has an initial crack at $x=0$ with the crack length of $a_{0}=60 \times 10^{-3} \mathrm{~m}$. The plate has the elastic modulus of $E=2 \times 10^{11} \mathrm{~N} / \mathrm{m}^{2}$, Poisson's ratio of $v=0.3$, and the critical energy release rate of $G_{c}=255 \times 10^{3} \mathrm{~J} / \mathrm{m} 2$ [71]. The plate is fixed at two edges $x=-L / 2$ and $x=L / 2$, and it is subjected to incremental vertical displacements at two points located at $\left(x= \pm 3.3333 \times 10^{-4} \mathrm{~m}, y=6.6667 \times 10^{-4} \mathrm{~m}\right)$.

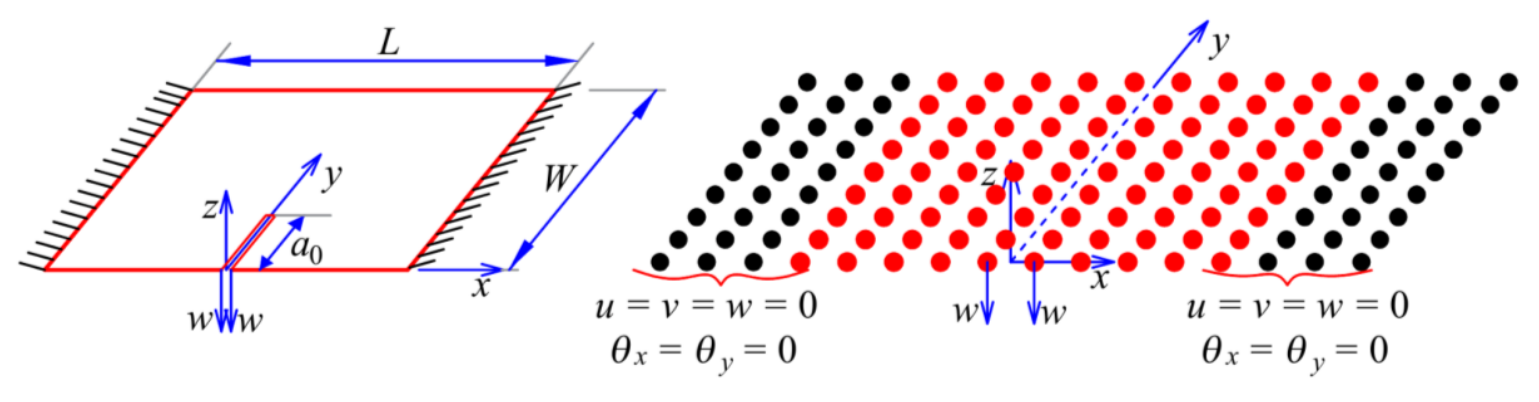

(a)

(b)

Fig. 25. Plate subjected to stretching and tearing (a): geometry, (b): PD discretized model

In the PD model, the plate is uniformly discretized with the mesh size of $\Delta x=6.6667 \times 10^{-4} \mathrm{~m}$ . To apply boundary conditions, three fictitious layers of material points are added on the left and the right sides of the plate. All DOFs of the fictitious material points as well as DOFs of 
the material points located at $x=-L / 2$ and $x=L / 2$ are set equal to zero. To apply the loading conditions, material points located at $\left(x=3.3333 \times 10^{-4} \mathrm{~m}, y=6.6667 \times 10^{-4} \mathrm{~m}\right)$ and $\left(x=-3.3333 \times 10^{-4} \mathrm{~m}, y=6.6667 \times 10^{-4} \mathrm{~m}\right)$ are applied incremental displacements as $w=0.002 \times i_{l}$, with $i_{l}=1, \ldots, 42$ represents the load step number. At each load step, the quasistatic solution is obtained for 18000 iterations by using the ADR method [68, 69]. The numerical procedure for this problem is shown in Fig. 26.

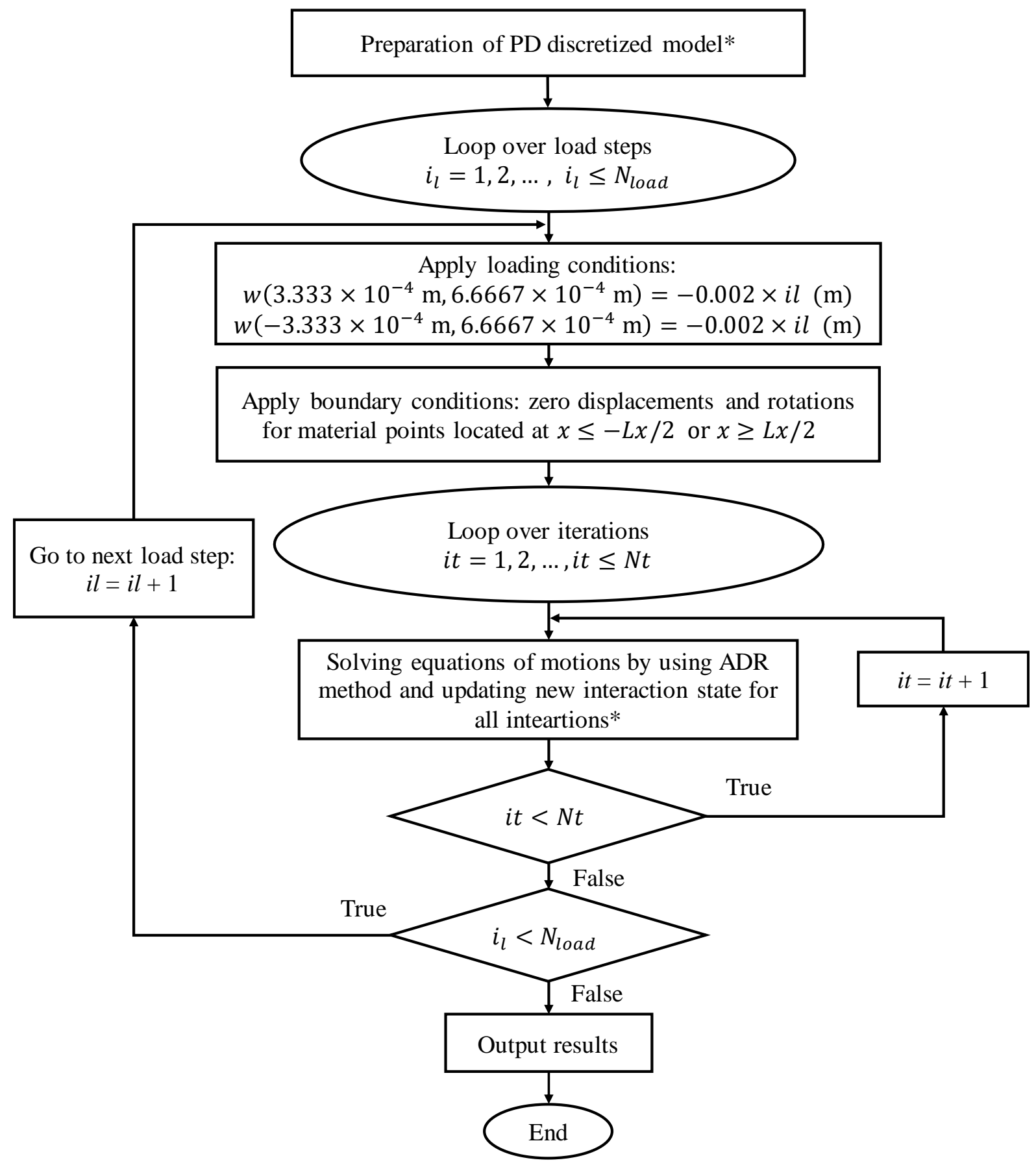

Fig. 26. Numerical procedure for the problem of a plate subjected to stretching and tearing (*see Fig. 10 for the preparation of the PD discretized model)

Fig. 27-Fig. 30 show the damage evolution on the plate. It can be observed from the figures that when the vertical displacements are continuously increased, the crack propagates along the 
positive $y$-direction. When the applied displacement is $w=0.084 \mathrm{~m}$, the crack propagates to the location of nearly $(x=0, y=0.2 \mathrm{~m}, z=0)$ as shown in Fig. 30. Moreover, larger crack openings are observed due to the larger deformations. This observation agrees well with the experimental results in [70] and the numerical results in [71, 72].

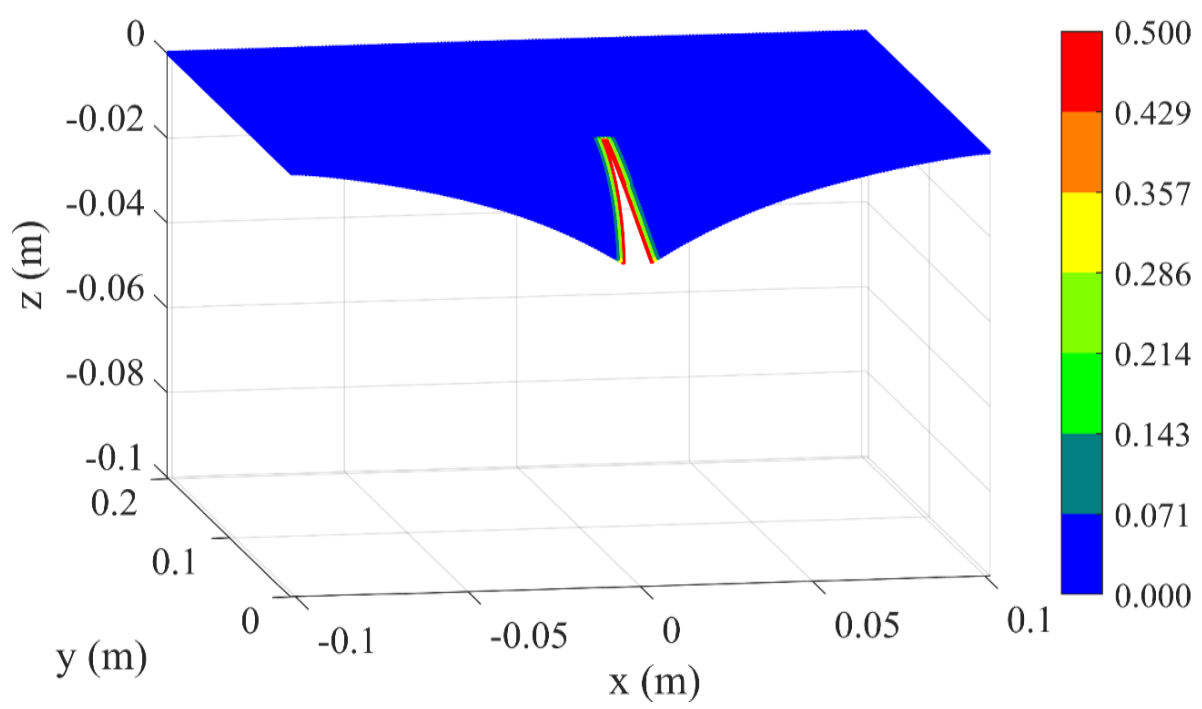

Fig. 27. Damage on the plate when the applied displacement $w=0.024 \mathrm{~m}$

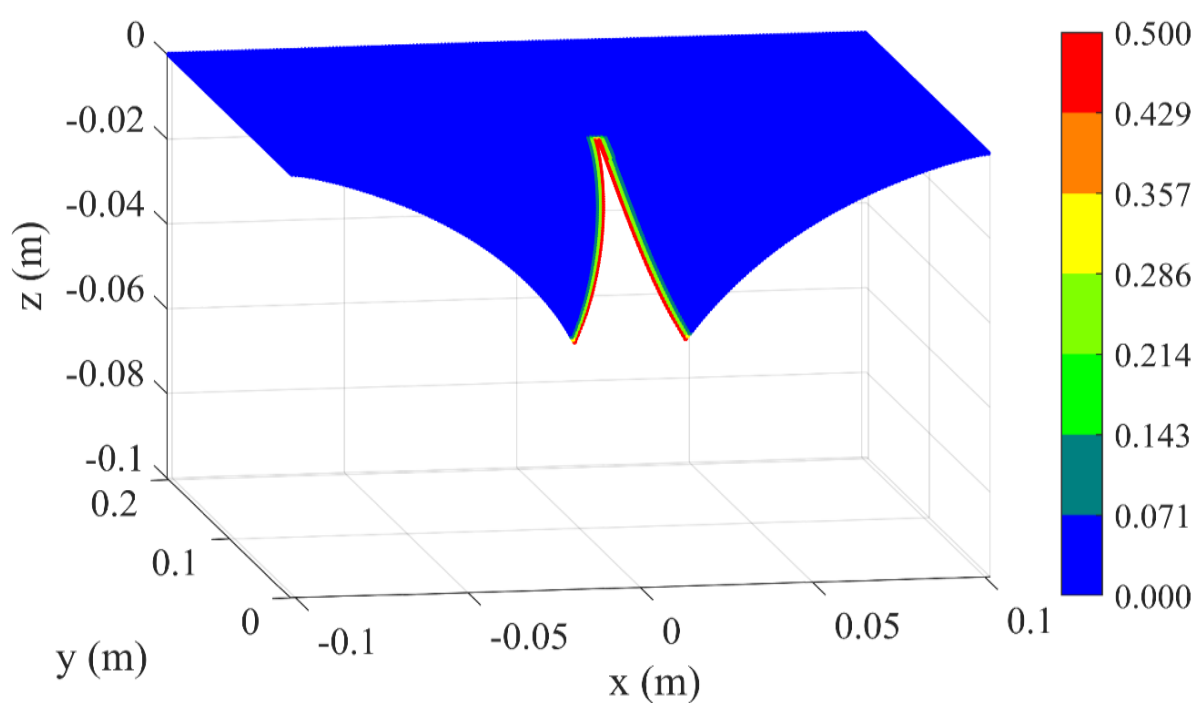

Fig. 28. Damage on the plate when the applied displacement $w=0.044 \mathrm{~m}$ 


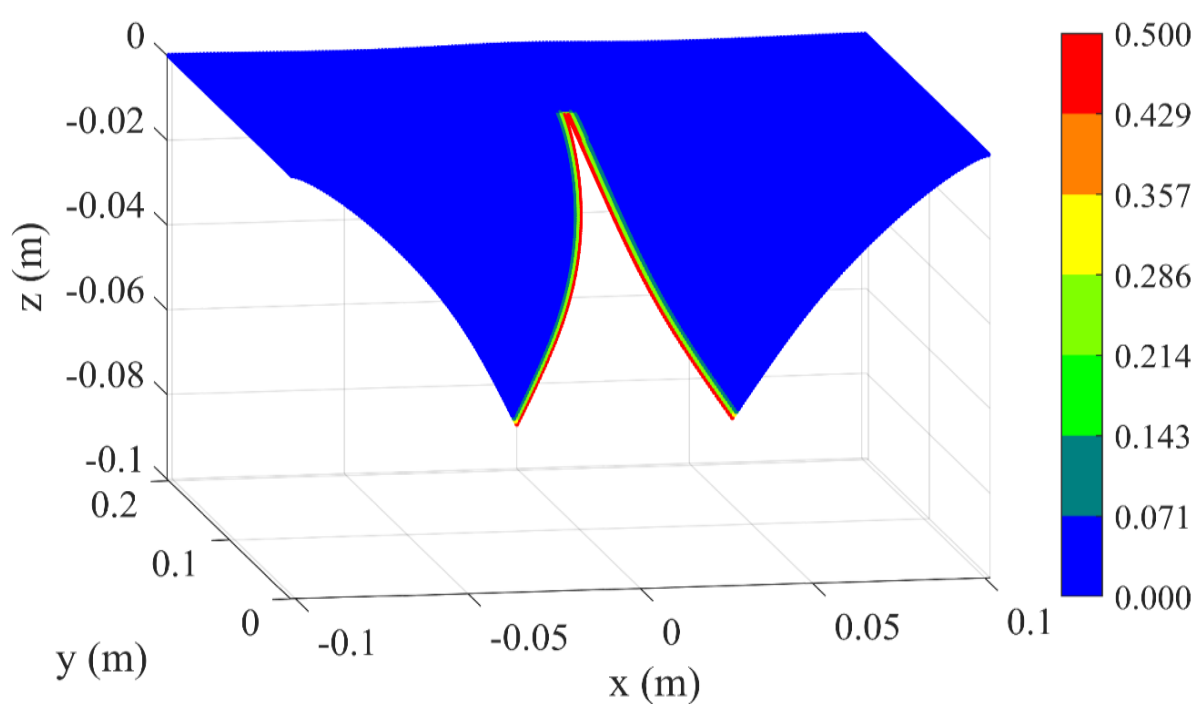

Fig. 29. Damage on the plate when the applied displacement $w=0.064 \mathrm{~m}$

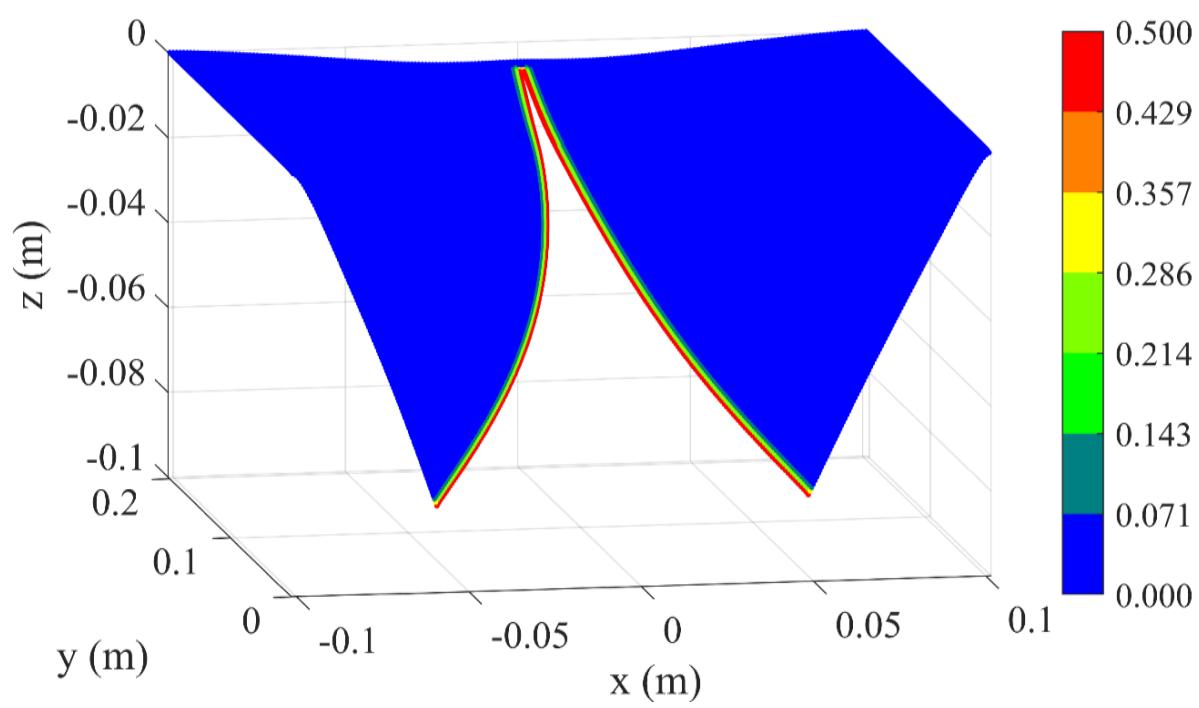

Fig. 30. Damage on the plate when the applied displacement $w=0.084 \mathrm{~m}$

Fig. 31 shows the history of the equivalent applied forces versus the applied vertical displacements. At each load step, after 18000 iterations for the ADR solution, the equivalent applied forces on two material points located at $\left(x=3.3333 \times 10^{-4} \mathrm{~m}, y=6.6667 \times 10^{-4} \mathrm{~m}\right)$ and $\left(x=-3.3333 \times 10^{-4} \mathrm{~m}, y=6.6667 \times 10^{-4} \mathrm{~m}\right)$ are calculated as

$$
\begin{aligned}
& F_{z(i)}=\frac{{ }^{0} V_{(i)}}{h} \sum_{j=1}^{N} \mu_{(i)(j)} \overline{\mathbf{f}}_{(i)(j)}{ }^{0} V_{(j)} \\
& F_{z(l)}=\frac{{ }^{0} V_{(l)}}{h} \sum_{j=1}^{N} \mu_{(l)(m)} \overline{\mathbf{f}}_{(l)(m)}{ }^{0} V_{(m)}
\end{aligned}
$$


where $i$ and $l$ are the material points located at $\left(x=3.3333 \times 10^{-4} \mathrm{~m}, y=6.6667 \times 10^{-4} \mathrm{~m}\right)$ and $\left(x=-3.3333 \times 10^{-4} \mathrm{~m}, y=6.6667 \times 10^{-4} \mathrm{~m}\right)$, respectively. Material point $j$ is one of the family members of material point $i$. Meanwhile, material point $m$ is one of the family members of material point $l$. The equivalent applied forces shown in Fig. 31 is the average values of the forces calculated by using Eq. (67).

As can be observed from Fig. 31, the equivalent applied forces versus the applied displacements captured by the nonlinear PD model has a good agreement with the experimental results given in [70]. When the applied displacements are smaller than $0.06 \mathrm{~m}$, the PD and the experimental results have a very good agreement. However, when the applied displacements are higher than $0.06 \mathrm{~m}$, there are small differences between the calculated forces in PD and the experimental values. These differences can be caused by plastic deformation effects, which are not considered in the PD simulation.

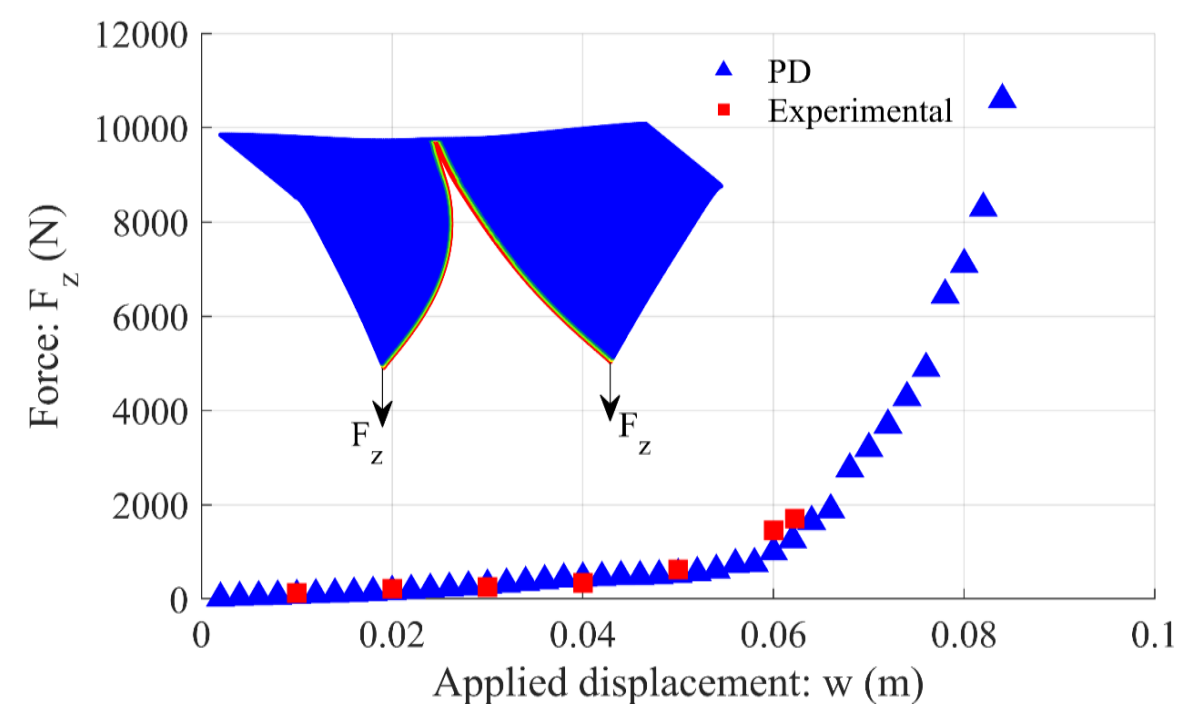

Fig. 31. Equivalent applied forces versus the applied displacements

\subsection{Tearing a plate}

In this section, another example of a plate subjected to tearing is investigated as shown in Fig. 32. The plate has the dimensions of $L=0.06 \mathrm{~m}, W=2 L$, and thickness of $h=5 \times 10^{-4} \mathrm{~m}$. The plate has two initial cracks located at $x=0.01 \mathrm{~m}$ and $x=-0.01 \mathrm{~m}$. The initial crack lengths are $a=0.03 \mathrm{~m}$. The material has an elastic modulus of $E=5.96 \mathrm{GPa}$, Poisson's ratio of $v=0.2$, and energy release rate of $G_{c}=8.8 \times 10^{3} \mathrm{~J} / \mathrm{m}^{2}[73]$.

Three edges of the plate are fixed and one edge is free as shown in Fig. 32. In PD, the model is uniformly discretized with the mesh size $\Delta x=L / 200$. To apply the boundary conditions, three fictitious layers of material points are added on each fixed edge. All DOFs of the fictitious material points as well as material points located along the fixed edges of the plate at $x= \pm L / 2$ and $y=W$ are set equal to zero.

The plate shown in Fig. 32 is subjected to incremental vertical displacements at the location of $(-0.01 \mathrm{~m} \leq x \leq 0.01 \mathrm{~m}, y=0, z=0)$. In the peridynamic model, the material points located at $(-0.01 \mathrm{~m} \leq x \leq 0.01 \mathrm{~m}, y=0, z=0)$ are subjected to incremental displacements as $w=0.001 \times i_{l}, i_{l}=1, \ldots, 60$, where $i_{l}$ represents the load step number. Similar to the previous example, at each load step, the quasi-static solution is obtained for 10000 iterations by using 
the ADR method. The same numerical procedure, except loading condition, shown in Fig. 26 is used for this problem for the given loading condition.

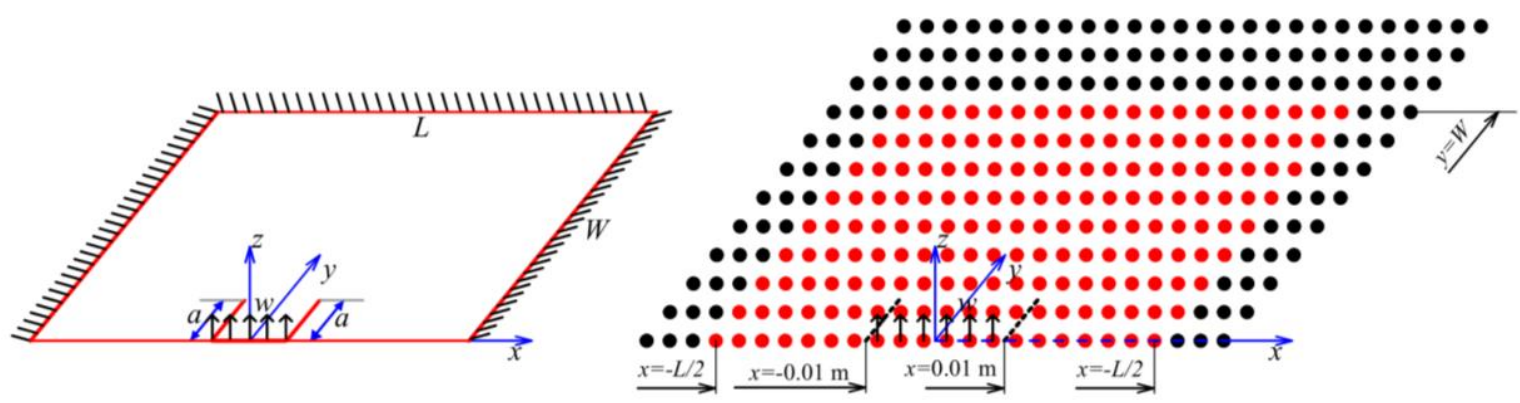

(a)

(b)

Fig. 32. Tearing a plate (a): geometry, (b): PD discretized model

Fig. 33-Fig. 35 show the damage evolution on the plate. As shown in Fig. 33, the cracks start propagating when the applied displacement is $w=0.025 \mathrm{~m}$. As the applied displacements are continuously increased, the cracks propagate diagonally as shown in Fig. 34. When the applied displacement is $w=0.033 \mathrm{~m}$, two cracks meet each other at $(x=0.039 \mathrm{~m}, y=0)$ as shown in Fig. 35. In Fig. 35, the final damage of the plate is shown in both deformed and undeformed configurations. It is observed that the damage pattern captured by the developed nonlinear PD model is similar to the numerical results obtained by Silling and Bobaru [55].

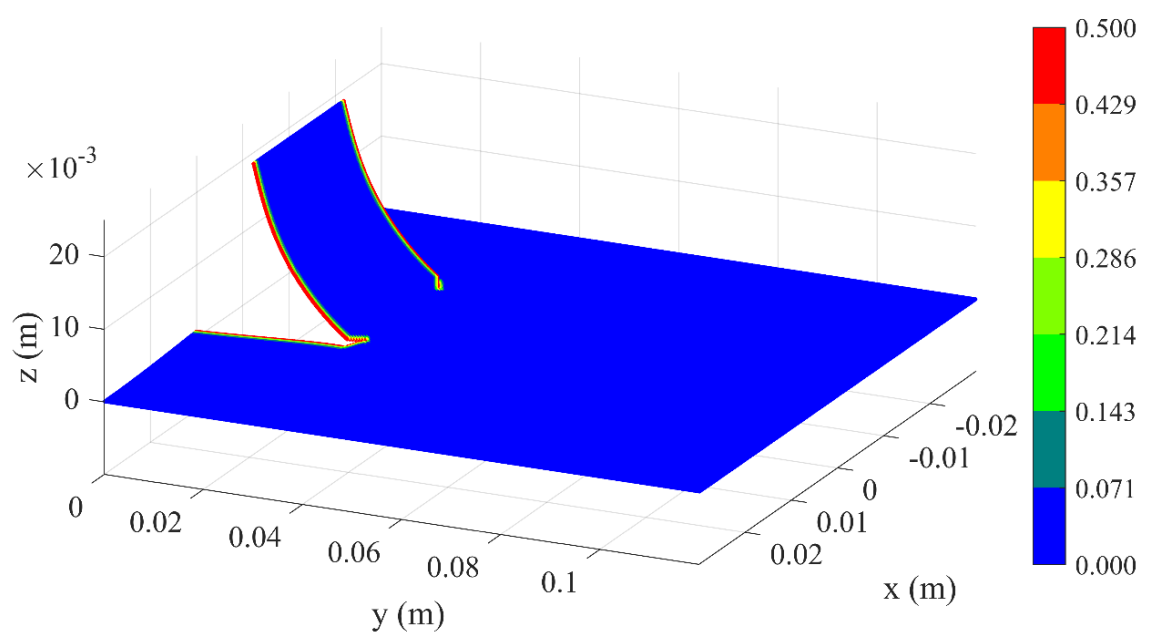

Fig. 33. Damage on the plate when the applied displacement $w=0.025 \mathrm{~m}$ 


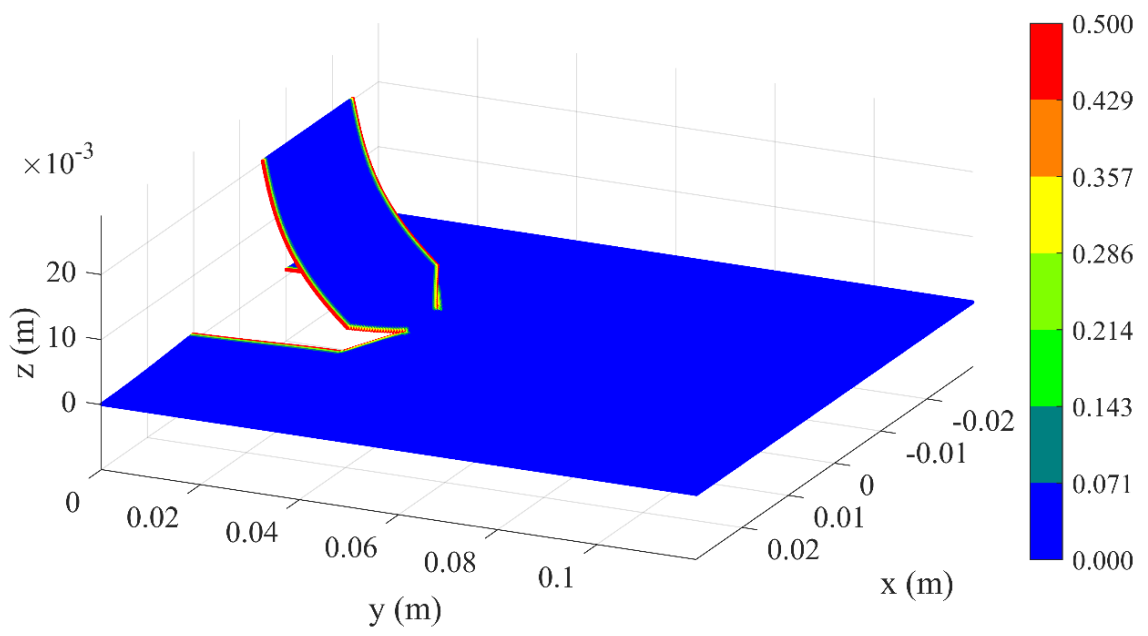

Fig. 34. Damage on the plate when the applied displacement $w=0.029 \mathrm{~m}$

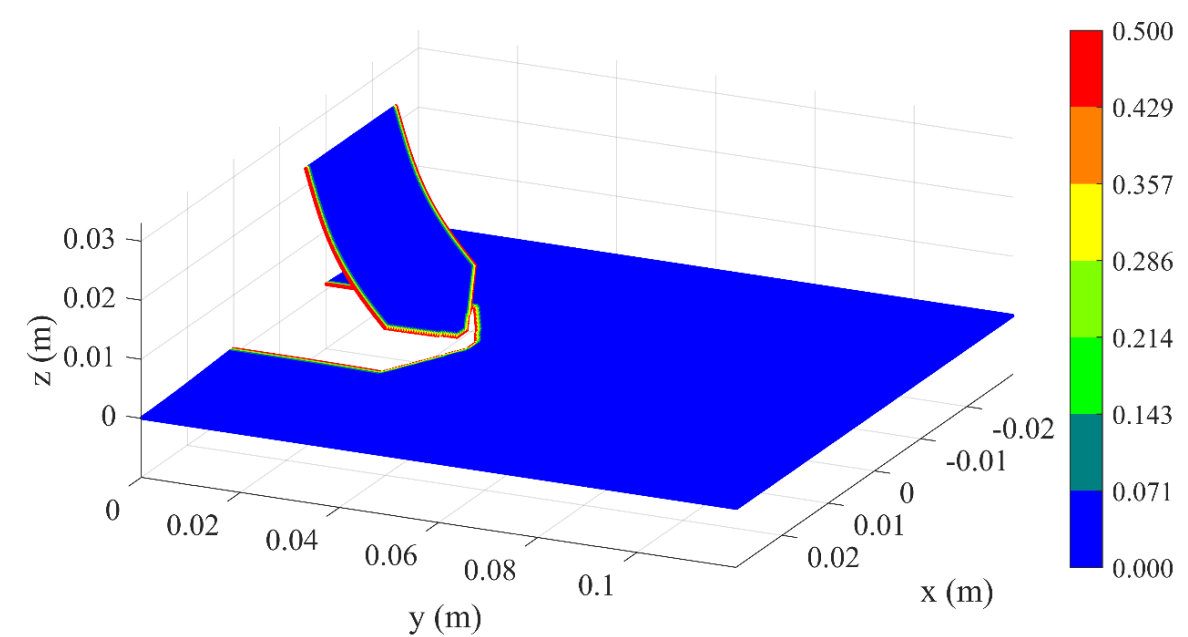

(a)

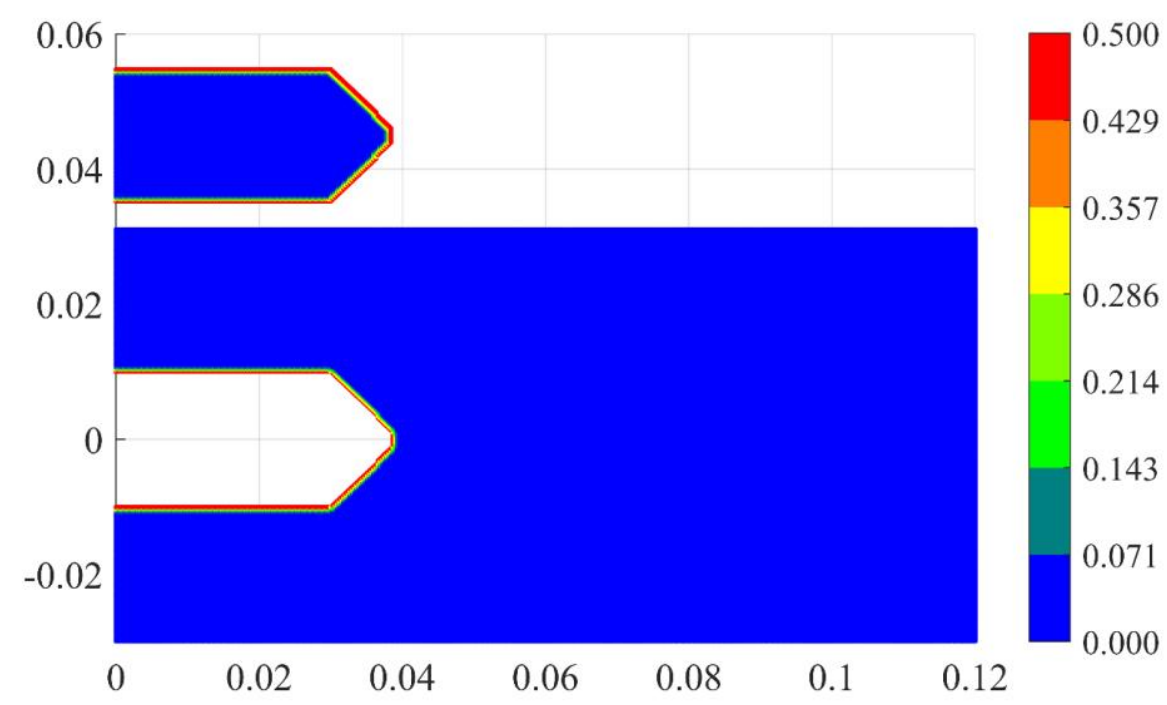

(b)

Fig. 35. Damage on the plate when the applied displacement $w=0.033 \mathrm{~m}$, (a): 3D view in deformed configuration, (b): 2D view in the undeformed configuration 


\subsection{Plate subjected to torsion}

In this section, damages on a plate subjected to torsional loading are investigated as shown in Fig. 36. The plate has dimensions of $L=0.3 \mathrm{~m}, W=0.127 \mathrm{~m}$, and thickness of $h=6 \times 10^{-3} \mathrm{~m}$ . The plate has an initial crack with a length of $a_{0}=26 \times 10^{-3} \mathrm{~m}$ as shown in Fig. 36. The material has the elastic modulus of $E=2 \times 10^{11} \mathrm{~N} / \mathrm{m}^{2}$ and Poisson's ratio of $v=0.3$. The critical energy release rate is $G_{c}=22295 \mathrm{~J} / \mathrm{m}^{2}$.

Two edges of the plate are attached with rigid parts, shown in black in Fig. 36(a), and they are applied incremental rotations as

$$
\begin{aligned}
& \theta_{x(\text { left })}=-10^{-7} \times i t(\mathrm{rad}), \text { it }=1, \ldots, 10^{7} \\
& \theta_{x(\text { right })}=10^{-7} \times i t(\mathrm{rad}), \text { it }=1, \ldots, 10^{7}
\end{aligned}
$$

In PD, the model is uniformly discretized with a mesh size of $\Delta x=L / 100$ and the quasi-static solution is obtained by using the ADR method. To apply loading conditions, three fictitious layers of material points are added on the left and right sides of the plate as shown in Fig. 36(b). The rotation boundary conditions given in Eq. (68) are implemented by using displacement boundary conditions as

$\left[\begin{array}{lll}u & v & w\end{array}\right]^{T}=\mathbf{R x}-\mathbf{x}$

where $\mathbf{R}$ is the rotation matrix. Therefore, the displacements of the fictitious material points on the left side are implemented as

$\left[\begin{array}{c}u_{(k)} \\ v_{(k)} \\ w_{(k)}\end{array}\right]=\left[\begin{array}{ccc}1 & 0 & 0 \\ 0 & \cos \theta_{x(\mathrm{left})} & -\sin \theta_{x(\mathrm{left})} \\ 0 & \sin \theta_{x(\mathrm{left})} & \cos \theta_{x(\mathrm{left})}\end{array}\right]\left[\begin{array}{c}x_{(k)} \\ y_{(k)} \\ z_{(k)}\end{array}\right]-\left[\begin{array}{c}x_{(k)} \\ y_{(k)} \\ z_{(k)}\end{array}\right]$

Meanwhile, the displacements of the fictitious material points on the right side are implemented as

$\left[\begin{array}{c}u_{(j)} \\ v_{(j)} \\ w_{(j)}\end{array}\right]=\left[\begin{array}{ccc}1 & 0 & 0 \\ 0 & \cos \theta_{x(\text { right })} & -\sin \theta_{x(\text { right })} \\ 0 & \sin \theta_{x(\text { right })} & \cos \theta_{x(\text { right })}\end{array}\right]\left[\begin{array}{c}x_{(j)} \\ y_{(j)} \\ z_{(j)}\end{array}\right]-\left[\begin{array}{c}x_{(j)} \\ y_{(j)} \\ z_{(j)}\end{array}\right]$

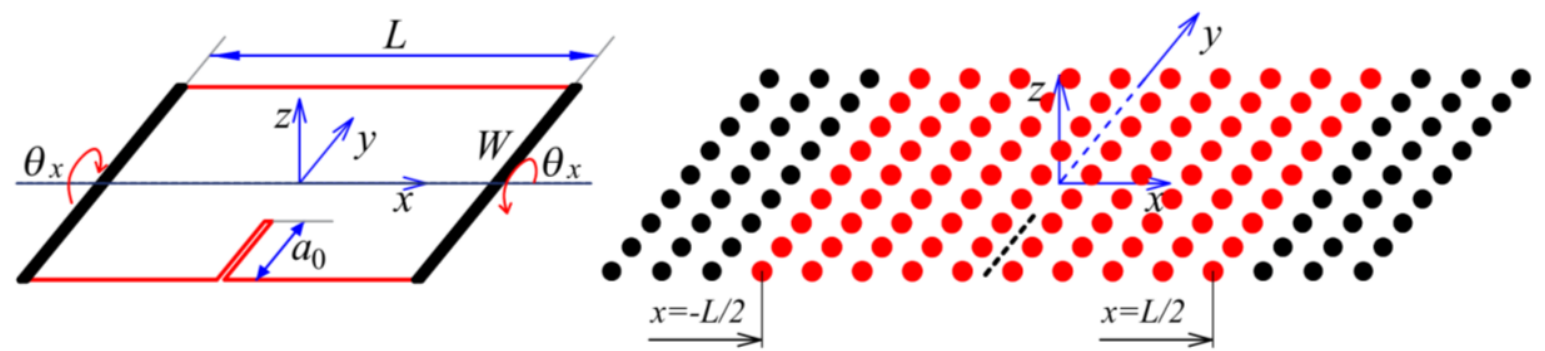

(a)

(b)

Fig. 36. Plate subjected to torsion 
Fig. 37-Fig. 39 show the damage evolution on the plate subjected to torsion. As shown in Fig. 37 , the crack starts propagating when the applied rotational angle is $\left|\theta_{x}\right|=0.082$ (rad). As the rotation $\left|\theta_{x}\right|$ is increased, the crack propagates along the positive $y$ direction and reaches the final damage location at $(x=0, y=0.125 \mathrm{~m})$ when the applied rotational angle reaches $\left|\theta_{x}\right|=0.094$ (rad) as shown in Fig. 39. It can also be observed that the damage pattern captured by the nonlinear PD agrees well with the results captured by Zavattieri [74].

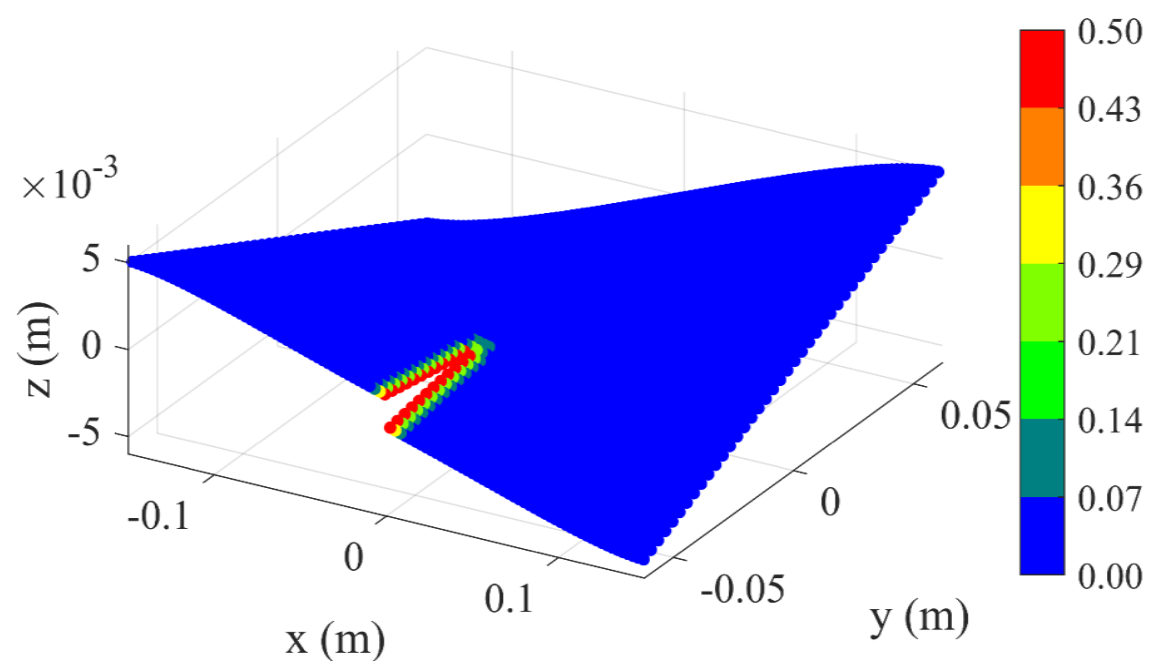

Fig. 37. Damage on the plate when $\left|\theta_{x}\right|=0.082(\mathrm{rad})$

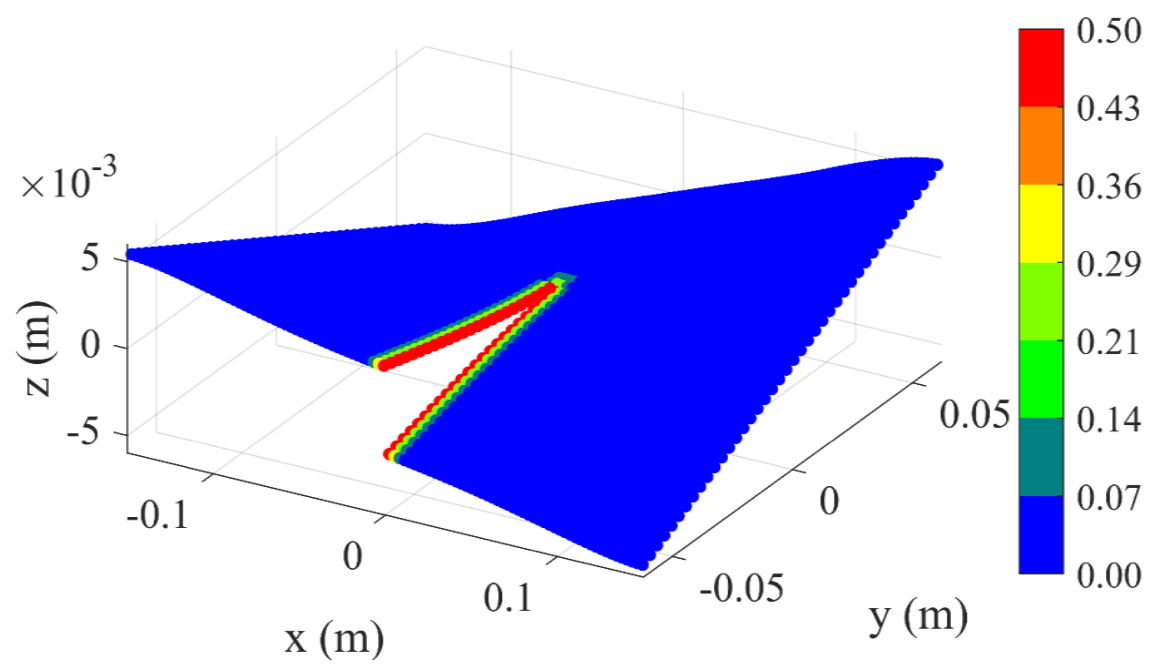

Fig. 38. Damage on the plate when $\left|\theta_{x}\right|=0.088(\mathrm{rad})$ 


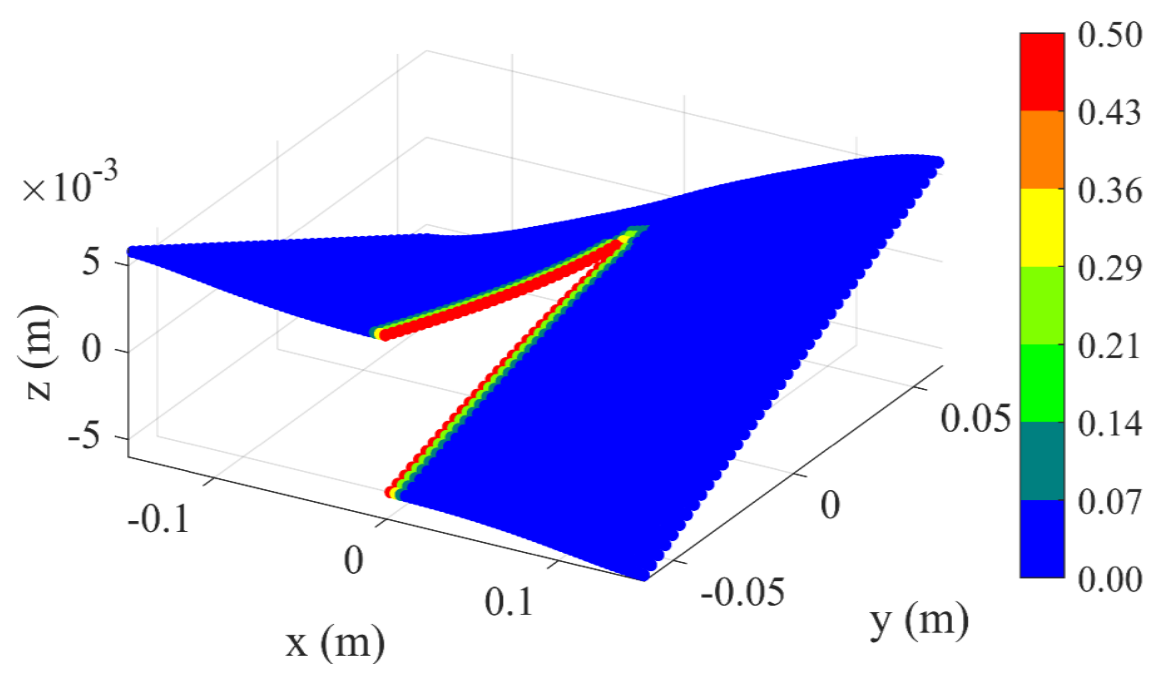

Fig. 39. Damage on the plate when $\left|\theta_{x}\right|=0.094(\mathrm{rad})$

Fig. 40 shows the variations of the crack length versus the rotational angles. As it can be observed from the figure, when the rotational angle is smaller than 0.08 (rad), the crack growth is quite slow. Beyond this limit, the crack starts growing much faster and reaches the final length of $0.125 \mathrm{~m}$ when the applied rotational angle is $\left|\theta_{x}\right|=0.094(\mathrm{rad})$.

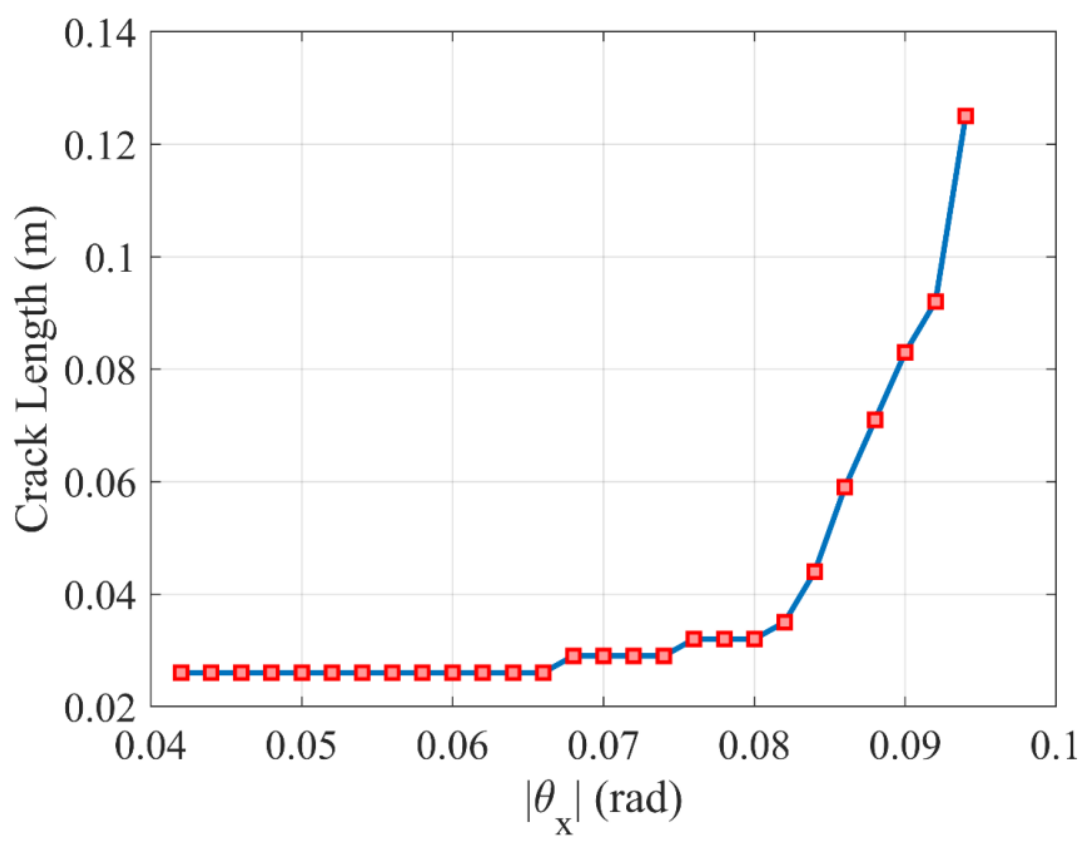

Fig. 40. Crack length $(\mathrm{m})$ versus rotational angle (rad)

\section{Conclusion}

In this study, a novel nonlinear peridynamic model for predicting large deformations of plates is introduced. The energy-based damage criterion and the numerical procedure for damage prediction by using the proposed nonlinear PD model are also provided.

The accuracy of the nonlinear PD model is verified by comparing the PD results with the nonlinear FEA results for the problem of a plate subjected bending as well as a plate subjected 
to vertical shear forces. After the verification, the nonlinear PD model is used to predict progressive damages on a plate subjected to out-of-plane stretching and tearing, a plate with two parallel cracks subjected to tearing, and a plate with a pre-existing crack subjected to torsional loading. The predicted results in terms of damage patterns agree very well with the observations from the experiments and previous numerical studies. The proposed nonlinear PD can be further applied to predict possible damages on plates and shell structures during their operation process.

\section{Acknowledgment}

The authors gratefully acknowledge the support from the Ministry of Education and Training in Vietnam and the University of Strathclyde. Results were obtained using the ARCHIE-WeSt High Performance Computer (www.archie-west.ac.uk) based at the University of Strathclyde. 


\section{Appendix A. PD constants for in-plane deformations}

In this section, the derivations to obtain the PD constants for in-plane deformations are presented. First, let $k$ and $j$ be two material points in a two-dimensional structure. By using Taylor's series expansion, a variable $f\left({ }^{0} x_{(j)},{ }^{0} y_{(j)}\right)$ at material point $j$ can be expressed in term of the variable $f\left({ }^{0} x_{(k)},{ }^{0} y_{(k)}\right)$ at material point $k$ as

$$
\begin{aligned}
f\left({ }^{0} x_{(j)},{ }^{0} y_{(j)}\right)= & f\left({ }^{0} x_{(k)},{ }^{0} y_{(k)}\right)+\left[{ }^{0} x_{(j)}-{ }^{0} x_{(k)}\right] \frac{\partial}{\partial^{0} x}\left(f\left({ }^{0} x_{(k)},{ }^{0} y_{(k)}\right)\right) \\
& +\left[{ }^{0} y_{(j)}-{ }^{0} y_{(k)}\right] \frac{\partial}{\partial^{0} y}\left(f\left({ }^{0} x_{(k)},{ }^{0} y_{(k)}\right)\right)+\cdots
\end{aligned}
$$

or

$$
f\left({ }^{0} x_{(j)},{ }^{0} y_{(j)}\right)=f\left({ }^{0} x_{(k)},{ }^{0} y_{(k)}\right)+{ }_{0} f_{, x(k)}\left[{ }^{0} x_{(j)}-{ }^{0} x_{(k)}\right]+{ }_{0} f_{, y(k)}\left[{ }^{0} y_{(j)}-{ }^{0} y_{(k)}\right]+\cdots
$$

with

$$
{ }_{0} f_{, x(k)}=\frac{\partial}{\partial^{0} x}\left(f\left({ }^{0} x_{(k)},{ }^{0} y_{(k)}\right)\right)
$$

and

$$
{ }_{0} f_{, y(k)}=\frac{\partial}{\partial^{0} y}\left(f\left({ }^{0} x_{(k)},{ }^{0} y_{(k)}\right)\right)
$$

In Eq. (A1), the parameter $f$ can be any variable. For instance, $f$ can be the displacements and rotations of material points.

If $k$ and $j$ are two different material points $(k \neq j)$, the distance between them in the undeformed configuration is nonzero $\left({ }^{0} \xi_{(k)(j)} \neq 0\right)$. Therefore, Eq. (A1b) can be rewritten as

$$
\frac{f\left({ }^{0} x_{(j)},{ }^{0} y_{(j)}\right)-f\left({ }^{0} x_{(k)},{ }^{0} y_{(k)}\right)}{{ }^{0} \xi_{(k)(j)}}={ }_{0} f_{, x(k)} \frac{{ }^{0} x_{(j)}-{ }^{0} x_{(k)}}{{ }^{0} \xi_{(k)(j)}}+{ }_{0} f_{, y(k)} \frac{{ }^{0} y_{(j)}-{ }^{0} y_{(k)}}{{ }^{0} \xi_{(k)(j)}}+\cdots
$$

By using the relations given in Eq. (43), Eq. (A2) can be rewritten as

$$
\frac{f\left({ }^{0} x_{(j)},{ }^{0} y_{(j)}\right)-f\left({ }^{0} x_{(k)},{ }^{0} y_{(k)}\right)}{{ }^{0} \xi_{(k)(j)}}={ }_{0} f_{, x(k)} \cos \varphi+{ }_{0} f_{, y(k)} \sin \varphi+\cdots
$$

where the angle $\varphi$ is shown in Fig. 6.

Replacing $f$ variable in Eq. (A3) by displacements and rotations at time $t$ of material points $k$ and $j$ results in the following relations

$$
\frac{{ }^{t} u_{(j)}-{ }^{t} u_{(k)}}{{ }^{0} \xi_{(k)(j)}}={ }_{0}^{t} u_{, x(k)} \cos \varphi+{ }_{0}^{t} u_{, y(k)} \sin \varphi+\cdots
$$




$$
\begin{aligned}
& \frac{{ }^{t} v_{(j)}-{ }^{t} v_{(k)}}{{ }^{0} \xi_{(k)(j)}}={ }_{0}^{t} v_{, x(k)} \cos \varphi+{ }_{0}^{t} v_{, y(k)} \sin \varphi+\cdots \\
& \frac{{ }^{t} w_{(j)}-{ }^{t} w_{(k)}}{{ }^{0} \xi_{(k)(j)}}={ }_{0}^{t} w_{, x(k)} \cos \varphi+{ }_{0}^{t} w_{, y(k)} \sin \varphi+\cdots \\
& \frac{{ }^{t} \theta_{x(j)}-{ }^{t} \theta_{x(k)}}{{ }^{0} \xi_{(k)(j)}}={ }_{0}^{t} \theta_{x, x(k)} \cos \varphi+{ }_{0}^{t} \theta_{x, y(k)} \sin \varphi+\cdots \\
& \frac{{ }^{t} \theta_{y(j)}-{ }^{t} \theta_{y(k)}}{{ }^{0} \xi_{(k)(j)}}={ }_{0}^{t} \theta_{y, x(k)} \cos \varphi+{ }_{0}^{t} \theta_{y, y(k)} \sin \varphi+\cdots
\end{aligned}
$$

Similarly, replacing $f$ in Eq. (A3) by incremental displacements and incremental rotations (from time $t$ to time $t+\Delta t$ ) of material points $k$ and $j$ results in the following relations

$$
\begin{aligned}
& \frac{\underline{u}_{(j)}-\underline{u}_{(k)}}{{ }^{0} \xi_{(k)(j)}}={ }_{0} \underline{u}_{, x(k)} \cos \varphi+{ }_{0} \underline{u}_{, y(k)} \sin \varphi+\cdots \\
& \frac{\underline{v}_{(j)}-\underline{v}_{(k)}}{{ }^{0} \xi_{(k)(j)}}={ }_{0} \underline{v}_{, x(k)} \cos \varphi+{ }_{0} \underline{v}_{, y(k)} \sin \varphi+\cdots \\
& \frac{\underline{w}_{(j)}-\underline{w}_{(k)}}{{ }^{0} \xi_{(k)(j)}}={ }_{0} \underline{w}_{, x(k)} \cos \varphi+{ }_{0} \underline{w}_{y(k)} \sin \varphi+\cdots \\
& \frac{\underline{\theta}_{x(j)}-\underline{\theta}_{x(k)}}{{ }^{0} \xi_{(k)(j)}}={ }_{0} \underline{\theta}_{x, x(k)} \cos \varphi+{ }_{0} \underline{\theta}_{x, y(k)} \sin \varphi+\cdots \\
& \frac{\underline{\theta}_{y(j)}-\underline{\theta}_{y(k)}}{{ }^{0} \xi_{(k)(j)}}={ }_{0} \underline{\theta}_{y, x(k)} \cos \varphi+{ }_{0} \underline{\theta}_{y, y(k)} \sin \varphi+\cdots
\end{aligned}
$$

Therefore, by using the relations given in Eqs. (A4-5), the bond stretches given in Eqs. (41bc) can be rewritten as

$$
\begin{aligned}
{ }_{0}^{t} s_{i p(k)(j)}= & { }_{0}^{t} u_{, x(k)} \cos ^{2} \varphi+{ }_{0}^{t} u_{, y(k)} \sin \varphi \cos \varphi+{ }_{0}^{t} v_{, x(k)} \sin \varphi \cos \varphi+{ }_{0}^{t} v_{, y(k)} \sin ^{2} \varphi \\
+ & \frac{1}{2}\left[\begin{array}{l}
\left({ }_{0}^{t} u_{, x(k)} \cos \varphi+{ }_{0}^{t} u_{, y(k)} \sin \varphi\right)^{2}+\left({ }_{0}^{t} v_{, x(k)} \cos \varphi+{ }_{0}^{t} v_{, y(k)} \sin \varphi\right)^{2} \\
+\left({ }_{0}^{t} w_{, x(k)} \cos \varphi+{ }_{0}^{t} w_{, y(k)} \sin \varphi\right)^{2}
\end{array}\right] \\
0 \underline{s}_{i p(k)(j)}= & { }_{0} \underline{u}_{, x(k)} \cos ^{2} \varphi+{ }_{0} \underline{u}_{, y(k)} \sin \varphi \cos \varphi+{ }_{0} \underline{v}_{, x(k)} \sin \varphi \cos \varphi+{ }_{0} \underline{v}, y(k) \sin ^{2} \varphi \\
& {\left[\begin{array}{l}
\left({ }_{0}^{t} u_{, x(k)} \cos \varphi+{ }_{0}^{t} u_{, y(k)} \sin \varphi\right)\left({ }_{0} \underline{u}_{, x(k)} \cos \varphi+{ }_{0} \underline{u}_{, y(k)} \sin \varphi\right) \\
+\left({ }_{0}^{t} v_{, x(k)} \cos \varphi+{ }_{0}^{t} v_{, y(k)} \sin \varphi\right)\left({ }_{0} \underline{v}_{, x(k)} \cos \varphi+{ }_{0} \underline{v}_{, y(k)} \sin \varphi\right) \\
+\left({ }_{0}^{t} w_{, x(k)} \cos \varphi+{ }_{0}^{t} w_{, y(k)} \sin \varphi\right)\left({ }_{0} \underline{w}_{, x(k)} \cos \varphi+{ }_{0} \underline{w}_{, y(k)} \sin \varphi\right)
\end{array}\right] }
\end{aligned}
$$


Therefore, by using the bond stretches given in Eq. (A6) and by disregarding the interactions beyond the horizon size of a material point, the dilatations given in Eqs. (41d-e) can be rewritten as

$$
\begin{aligned}
& { }_{0}^{t} \vartheta_{(k)}=d_{i p} h \int_{0}^{2 \pi} \int_{0}^{\delta}\left[\begin{array}{c}
{ }_{0}^{t} u_{, x(k)} \cos ^{2} \varphi+{ }_{0}^{t} u_{, y(k)} \sin \varphi \cos \varphi \\
+{ }_{0}^{t} v_{, x(k)} \sin \varphi \cos \varphi+{ }_{0}^{t} v_{, y(k)} \sin ^{2} \varphi \\
+\frac{1}{2}\left({ }_{0}^{t} u_{, x(k)} \cos \varphi+{ }_{0}^{t} u_{, y(k)} \sin \varphi\right)^{2} \\
+\frac{1}{2}\left({ }_{0}^{t} v_{, x(k)} \cos \varphi+{ }_{0}^{t} v_{, y(k)} \sin \varphi\right)^{2} \\
+\frac{1}{2}\left({ }_{0}^{t} w_{, x(k)} \cos \varphi+{ }_{0}^{t} w_{, y(k)} \sin \varphi\right)^{2}
\end{array}\right]{ }_{0}^{0} \xi_{(k)(j)} d \varphi \\
& \underline{\vartheta}_{0} \underline{\underline{v}}_{(k)}=d_{i p} h \int_{0}^{2 \pi} \int_{0}^{\delta}\left[\begin{array}{l}
{ }_{0} \underline{u}_{, x(k)} \cos ^{2} \varphi+{ }_{0} \underline{u}_{, y(k)} \sin \varphi \cos \varphi \\
+{ }_{0} \underline{\underline{v}}_{, x(k)} \sin \varphi \cos \varphi+{ }_{0} \underline{v}_{, y(k)} \sin ^{2} \varphi \\
+\left({ }_{0}^{t} u_{, x(k)} \cos \varphi+{ }_{0}^{t} u_{, y(k)} \sin \varphi\right)\left({ }_{0} \underline{u}_{, x(k)} \cos \varphi+{ }_{0} \underline{u}_{, y(k)} \sin \varphi\right) \\
+\left({ }_{0}^{t} v_{, x(k)} \cos \varphi+{ }_{0}^{t} v_{, y(k)} \sin \varphi\right)\left({ }_{0} \underline{\underline{v}}_{, x(k)} \cos \varphi+{ }_{0} \underline{v}_{, y(k)} \sin \varphi\right) \\
+\left({ }_{0}^{t} w_{, x(k)} \cos \varphi+{ }_{0}^{t} w_{, y(k)} \sin \varphi\right)\left({ }_{0} \underline{w}_{, x(k)} \cos \varphi+{ }_{0} \underline{w}_{, y(k)} \sin \varphi\right)
\end{array}\right]{ }^{0} \xi_{(k)(j)} d^{0} \xi_{(k)(j)} d \varphi
\end{aligned}
$$

By performing the integrations in Eq. (A7), the dilatations can be calculated as

$$
\begin{aligned}
& { }_{0}^{t} \vartheta_{(k)}=d_{i p} \frac{\pi h \delta^{2}}{2}\left[\left({ }_{0}^{t} u_{, x(k)}+{ }_{0}^{t} v_{, y(k)}\right)+\frac{1}{2}\left(\begin{array}{l}
{ }_{0}^{t} u_{, x(k)}^{2}+{ }_{0}^{t} u_{, y(k)}^{2}+{ }_{0}^{t} v_{, x(k)}^{2} \\
+{ }_{0}^{t} v_{, y(k)}^{2}+{ }_{0}^{t} w_{, x(k)}^{2}+{ }_{0}^{t} w_{, y(k)}^{2}
\end{array}\right)\right] \\
& { }_{0} \underline{\vartheta}_{(k)}=d_{i p} \frac{\pi h \delta^{2}}{2}\left[\begin{array}{l}
0 \underline{u}_{, x(k)}+{ }_{0} \underline{v}_{, y(k)}+{ }_{0} \underline{u}_{, x(k)}{ }_{0}^{t} u_{, x(k)}+{ }_{0} \underline{u}_{, y(k) 0}{ }_{0}^{t} u_{, y(k)} \\
+{ }_{0} \underline{v}_{, x(k)}{ }_{0}^{t} v_{, x(k)}+{ }_{0} \underline{v}_{, y(k)}{ }_{0}^{t} v_{, y(k)}+{ }_{0} \underline{w}_{, x(k)}{ }_{0}^{t} w_{, x(k)}+{ }_{0} \underline{w}_{, y(k)}{ }_{0}^{t} w_{, y(k)}
\end{array}\right]
\end{aligned}
$$

By comparing Eq. (A8) with Eqs. (34d-e), the PD constant for dilatations can be obtained as

$$
d_{i p}=\frac{2}{\pi h \delta^{2}}
$$

Similarly, by disregarding the interactions beyond the horizon size of material point $k$, the nonlinear strain energy per unit area for the in-plane deformations given in Eq. (41a) can be rewritten in the integral form as

$$
\bar{W}_{i p(k)}^{N L P D}=2 a_{i p}\left({ }_{0}^{t} \vartheta_{(k)}\right)\left({ }_{0} \underline{\vartheta}_{(k)}\right)+2 b_{i p} h \int_{0}^{2 \pi} \int_{0}^{\delta}\left({ }_{0}^{t} s_{i p(k)(j)}\right)\left({ }_{0} \underline{s}_{i p(k)(j)}\right){ }^{0} \xi_{(k)(j)}^{2} d^{0} \xi_{(k)(j)} d \varphi
$$

By utilizing the bond stretches in Eq. (A6) and by performing the integrations given in Eq. (A10), the nonlinear strain energy per unit area for the in-plane deformations can be calculated as 


$$
\begin{aligned}
& \bar{W}_{i p(k)}^{N L P D}=2 a_{i p 0}{ }^{t} \vartheta_{(k) 0} \underline{\vartheta}_{(k)}
\end{aligned}
$$

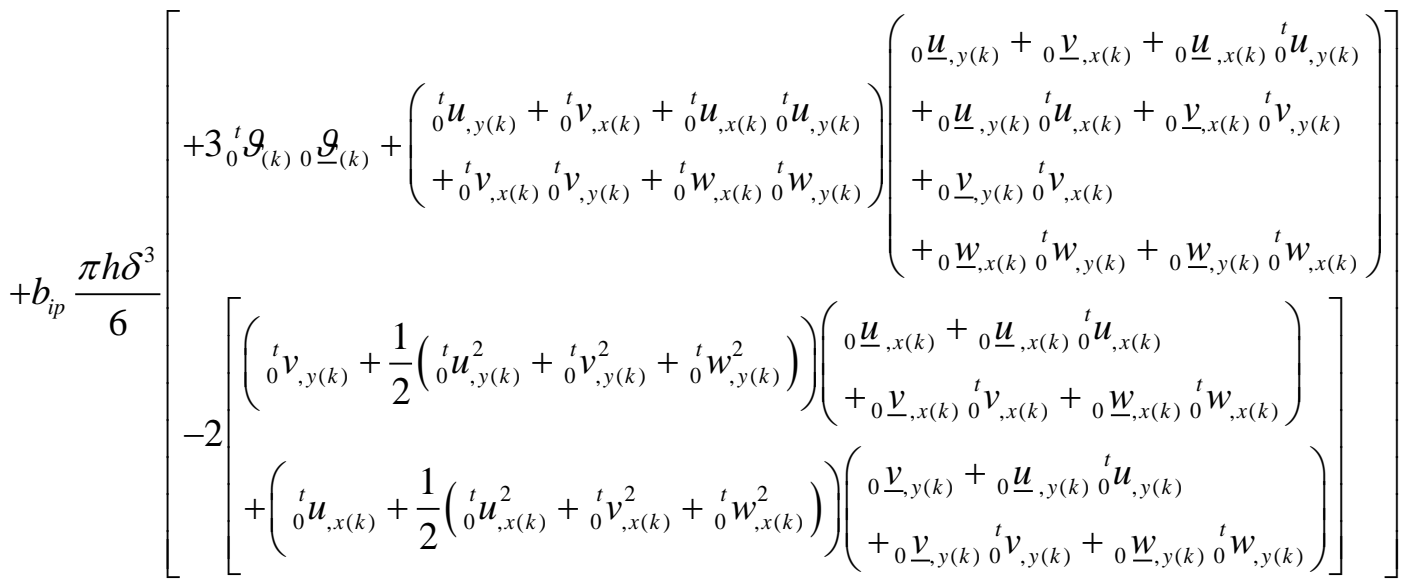

or

$$
\begin{aligned}
& \bar{W}_{i p(k)}^{N L P D}=\left(2 a_{i p}+b_{i p} \frac{\pi h \delta^{3}}{2}\right){ }_{0}^{t} \vartheta_{(k) 0} \underline{\vartheta}_{(k)}
\end{aligned}
$$

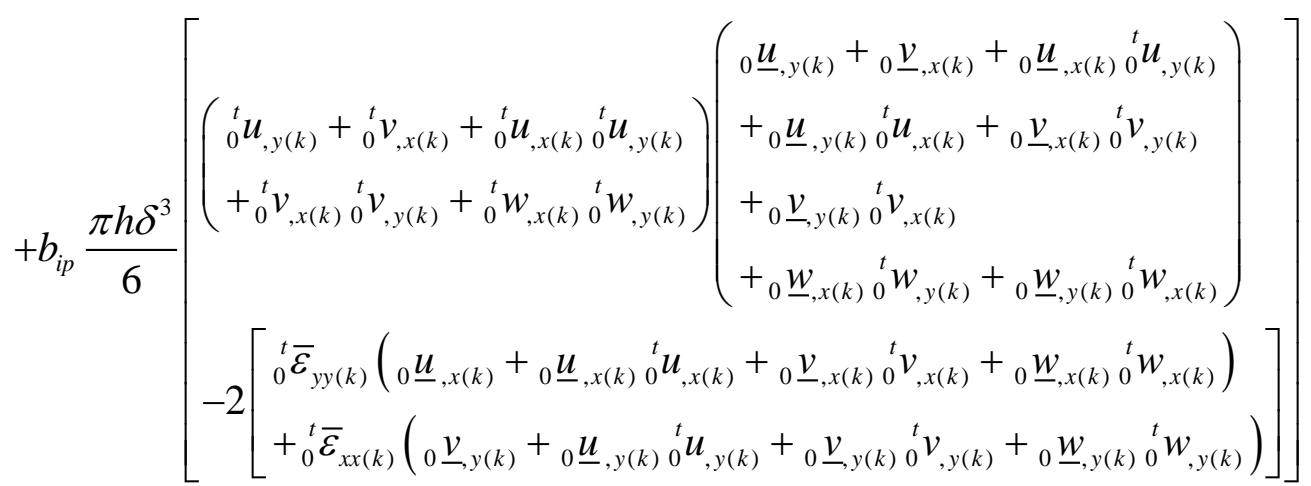

where ${ }_{0}^{t} \varepsilon_{x x}$ and ${ }_{0}^{t} \varepsilon_{y y}$ are defined in Eqs. (34b-c).

By comparing Eq. (A11b) with Eq. (35b), the following relations are obtained

$$
\begin{aligned}
& b_{i p} \frac{\pi h \delta^{3}}{6}=\frac{E h}{2(1+v)} \\
& a_{i p}+b_{i p} \frac{\pi h \delta^{3}}{2}=\frac{E h}{1-v^{2}}
\end{aligned}
$$

Therefore, by rearranging Eqs. (A12a-b), the PD constants for in-plane deformations can be obtained as

$$
\begin{aligned}
& b_{i p}=\frac{3 E}{(1+v) \pi \delta^{3}} \\
& a_{i p}=\frac{E h}{4\left(1-v^{2}\right)}(3 v-1)
\end{aligned}
$$




\section{Appendix B. PD constant for shear deformations}

In this section, the determination of the PD constant, $C_{s h}$, for shear deformations is presented. As shown in Fig. 7, ${ }^{t} \hat{\theta}_{(k)},{ }^{t} \hat{\theta}_{(j)}$ and $\underline{\hat{\theta}}_{(k)}, \underline{\hat{\theta}}_{(j)}$ are rotations and incremental rotations around the line of interaction between material points $k$ and $j$. The relative values of these rotations and incremental rotations represent the torsional angles (twisting angles) of the bond between material points $k$ and $j$. Therefore, by assuming the PD model is discretized with a fine mesh and the torsional deformations of the bond between material points $k$ and $j$ are insignificant, the approximation ${ }^{t} \hat{\theta}_{(k)} \approx{ }^{t} \hat{\theta}_{(j)}$ and $\underline{\hat{\theta}}_{(k)} \approx \underline{\hat{\theta}}_{(j)}$ can be assumed [27]. Thus, the nonlinear PD strain energy per unit area for the shear deformations given in Eq. (45a) can be rewritten as

$$
\bar{W}_{s h(k)}^{N L P D}=\frac{1}{2} C_{s h} \sum_{j=1}^{N}\left(\frac{{ }^{t} w_{(j)}-{ }^{t} w_{(k)}}{{ }^{0} \xi_{(k)(j)}}-{ }^{t} \hat{\theta}_{(k)}\right)\left(\frac{\underline{w}_{(j)}-\underline{w}_{(k)}}{{ }^{0} \xi_{(k)(j)}}-\underline{\hat{\theta}}_{(k)}\right){ }^{0} \xi_{(k)(j)}{ }^{0} V_{(j)}
$$

By using Eqs. (45b-c), the nonlinear PD strain energy per unit area for the shear deformations given in Eq. (B1) can be rewritten as

$$
\bar{W}_{s h(k)}^{N L P D}=\frac{1}{2} C_{s h} \sum_{j=1}^{N}\left(\begin{array}{l}
\frac{{ }^{t} w_{(j)}-{ }^{t} w_{(k)}}{{ }^{0} \xi_{(k)(j)}}+ \\
+\left(\sin ^{t} \theta_{y(k)}\right) \cos \varphi \\
-\left(\sin ^{t} \theta_{x(k)}\right) \sin \varphi
\end{array}\right)\left(\begin{array}{l}
\frac{\underline{w}_{(j)}-\underline{w}_{(k)}}{{ }^{0} \xi_{(k)(j)}}+ \\
+\theta_{y(k)} \cos \left({ }^{t} \theta_{y(k)}\right) \cos \varphi \\
-\theta_{x(k)} \cos \left({ }^{t} \theta_{x(k)}\right) \sin \varphi
\end{array}\right){ }^{0} \xi_{(k)(j)}{ }^{0} V_{(j)}
$$

By using the relation given in Eq. (A4c) and Eq. (A5c), and by disregarding the interactions beyond the horizon size of material point $k$, Eq. (B2) can be rewritten as

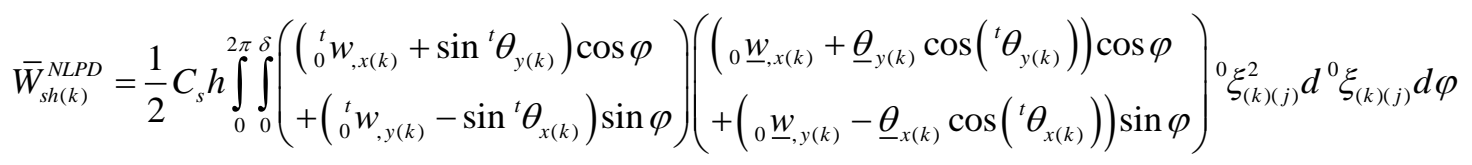

By performing the integrations in Eq. (B3), the nonlinear PD strain energy per unit area for the shear deformations can be obtained as

$$
\bar{W}_{s h(k)}^{N L P D}=\frac{1}{2} C_{s} \frac{\pi h \delta^{3}}{3}\left[\begin{array}{l}
\left({ }_{0}^{t} w_{, x(k)}+\sin ^{t} \theta_{y(k)}\right)\left({ }_{0} \underline{w}_{, x(k)}+\underline{\theta}_{y(k)} \cos \left({ }^{t} \theta_{y(k)}\right)\right) \\
+\left({ }_{0}^{t} w_{, y(k)}-\sin ^{t} \theta_{x(k)}\right)\left({ }_{0} \underline{w}_{y(k)}-\underline{\theta}_{x(k)} \cos \left({ }^{t} \theta_{x(k)}\right)\right)
\end{array}\right]
$$

By comparing Eq. (B4) with Eq. (35c), the PD constant for shear deformations can be obtained as

$$
C_{s}=\frac{3 k_{s} E}{(1+v) \pi \delta^{3}}
$$




\section{Appendix C. PD constants for bending deformations}

By using the relations given in Eqs. (A4d-e) and Eqs. (A5d-e), the bond stretches for bending deformations given in Eqs. (47d-e) can be rewritten as

$$
\begin{aligned}
& { }_{0}^{t} s_{b(k)(j)}=-{ }_{0}^{t} \theta_{y, x(k)} \cos ^{2} \varphi-{ }_{0}^{t} \theta_{y, y(k)} \sin \varphi \cos \varphi+{ }_{0}^{t} \theta_{x, x(k)} \sin \varphi \cos \varphi+{ }_{0}^{t} \theta_{x, y(k)} \sin ^{2} \varphi \\
& { }_{0} \underline{S}_{b(k)(j)}=-{ }_{0} \underline{\theta}_{y, x(k)} \cos ^{2} \varphi-{ }_{0} \underline{\theta}_{y, y(k)} \sin \varphi \cos \varphi+{ }_{0} \underline{\theta}_{x, x(k)} \sin \varphi \cos \varphi+{ }_{0} \underline{\theta}_{x, y(k)} \sin ^{2} \varphi
\end{aligned}
$$

By using Eq. (C1) and disregarding the interactions beyond the horizon of material point $k$, the terms given in Eqs. (47b-c) can be expressed as

$$
\begin{aligned}
& { }_{0}^{t} \vartheta_{b(k)}=d_{b} h \int_{0}^{2 \pi} \int_{0}^{\delta}\left(\begin{array}{l}
-{ }_{0}^{t} \theta_{y, x(k)} \cos ^{2} \varphi-{ }_{0}^{t} \theta_{y, y(k)} \sin \varphi \cos \varphi \\
+{ }_{0}^{t} \theta_{x, x(k)} \sin \varphi \cos \varphi+{ }_{0}^{t} \theta_{x, y(k)} \sin ^{2} \varphi
\end{array}\right){ }^{0} \xi_{(k)(j)} d^{0} \xi_{(k)(j)} d \varphi \\
& { }_{0} \underline{\vartheta}_{b(k)}=d_{b} h \int_{0}^{2 \pi} \int_{0}^{\delta}\left(\begin{array}{l}
-{ }_{0} \underline{\theta}_{y, x(k)} \cos ^{2} \varphi-{ }_{0} \underline{\theta}_{y, y(k)} \sin \varphi \cos \varphi \\
+{ }_{0} \underline{\theta}_{x, x(k)} \sin \varphi \cos \varphi+{ }_{0} \underline{\theta}_{x, y(k)} \sin ^{2} \varphi
\end{array}\right){ }_{0} \xi_{(k)(j)} d^{0} \xi_{(k)(j)} d \varphi
\end{aligned}
$$

Performing the integrations given in Eq. (C2) results in

$$
\begin{aligned}
& { }_{0}^{t} \vartheta_{b(k)}=d_{b} \frac{\pi h \delta^{2}}{2}\left(-{ }_{0}^{t} \theta_{y, x(k)}+{ }_{0}^{t} \theta_{x, y(k)}\right) \\
& { }_{0} \underline{\vartheta}_{b(k)}=d_{b} \frac{\pi h \delta^{2}}{2}\left(-{ }_{0} \underline{\theta}_{y, x(k)}+{ }_{0} \underline{\theta}_{x, y(k)}\right)
\end{aligned}
$$

By comparing Eq. (C3) with Eqs. (36c-d), the PD constant $d_{b}$ can be obtained as

$$
d_{b}=\frac{2}{\pi h \delta^{2}}
$$

Similarly, by using Eq. (C1) and by disregarding the interactions beyond the horizon of material point $k$, the nonlinear PD strain energy per unit area for bending formulations given in Eq. (47a) can be expressed as

$$
\begin{aligned}
& \bar{W}_{b(k)}^{N L P D}=2 a_{b}\left({ }_{0}^{t} \vartheta_{b(k)}\right)\left({ }_{0} \underline{\vartheta}_{b(k)}\right) \\
& +2 b_{b} h \int_{0}^{2 \pi} \int_{0}^{\delta}\left(\begin{array}{l}
-{ }_{0}^{t} \theta_{y, x(k)} \cos ^{2} \varphi-{ }_{0}^{t} \theta_{y, y(k)} \sin \varphi \cos \varphi \\
{ }_{0}^{t} \theta_{x, x(k)} \sin \varphi \cos \varphi+{ }_{0}^{t} \theta_{x, y(k)} \sin ^{2} \varphi
\end{array}\right)\left(\begin{array}{l}
-{ }_{0} \underline{\theta}_{y, x(k)} \cos ^{2} \varphi-{ }_{0} \underline{\theta}_{y, y(k)} \sin \varphi \cos \varphi \\
+{ }_{0} \underline{\theta}_{x, x(k)} \sin \varphi \cos \varphi+{ }_{0} \underline{\theta}_{x, y(k)} \sin ^{2} \varphi
\end{array}\right){ }^{0} \xi_{(k)(j)}^{2} d^{0} \xi_{(k)(j)} d \varphi
\end{aligned}
$$

By performing the integration in Eq. (C5), the nonlinear PD strain energy per unit area for bending formulations can be obtained as

$$
\bar{W}_{b(k)}^{N L P D}=2 a_{b}\left({ }_{0}^{t} \vartheta_{b(k)}\right)\left({ }_{0} \underline{\vartheta}_{b(k)}\right)+b_{b} \frac{\pi h \delta^{3}}{6}\left[\begin{array}{l}
3\left({ }_{0}^{t} \theta_{y, x(k)}-{ }_{0}^{t} \theta_{x, y(k)}\right)\left({ }_{0} \underline{\theta}_{y, x(k)}-{ }_{0} \underline{\theta}_{x, y(k)}\right) \\
+\left({ }_{0}^{t} \theta_{x, x(k)}-{ }_{0}^{t} \theta_{y, y(k)}\right)\left({ }_{0} \underline{\theta}_{x, x(k)}-{ }_{0} \underline{\theta}_{y, y(k)}\right) \\
+2{ }_{0}^{t} \theta_{x, y(k) 0} \underline{\theta}_{y, x(k)}+2{ }_{0}^{t} \theta_{y, x(k) 0} \underline{\theta}_{x, y(k)}
\end{array}\right]
$$

or 


$$
\bar{W}_{b(k)}^{N L P D}=2 a_{b}\left({ }_{0}^{t} \vartheta_{b(k)}\right)\left({ }_{0} \underline{\vartheta}_{b(k)}\right)+b_{b} \frac{\pi h \delta^{3}}{6}\left[\begin{array}{l}
3\left({ }_{0}^{t} \vartheta_{b(k)}\right)\left({ }_{0} \underline{\vartheta}_{b(k)}\right) \\
+\left({ }_{0}^{t} \theta_{x, x(k)}-{ }_{0}^{t} \theta_{y, y(k)}\right)\left({ }_{0} \underline{\theta}_{x, x(k)}-{ }_{0} \underline{\theta}_{y, y(k)}\right) \\
+2{ }_{0}^{t} \theta_{x, y(k) 0} \underline{\theta}_{y, x(k)}+2{ }_{0}^{t} \theta_{y, x(k) 0} \underline{\theta}_{x, y(k)}
\end{array}\right]
$$

Therefore, by comparing Eq. (C6b) with Eq. (36b), the following relations between PD constants for bending deformations and material constants can be obtained as

$b_{b} \frac{\pi h \delta^{3}}{6}=\frac{E h^{3}}{24(1+v)}$

$2 a_{b}+b_{b} \frac{\pi h \delta^{3}}{2}=\frac{E h^{3}}{12\left(1-v^{2}\right)}$

Therefore, the PD constants for bending deformations can be obtained as

$$
\begin{aligned}
& a_{b}=\frac{E h^{3}(3 v-1)}{48\left(1-v^{2}\right)} \\
& b_{b}=\frac{E h^{2}}{4(1+v) \pi \delta^{3}}
\end{aligned}
$$

\section{Appendix D. Adaptive relaxation method for nonlinear PD analyses of plate}

According to Underwood [68], Kilic and Madenci [69], the adaptive dynamic relaxation (ADR) can be used for static and quasi-static problems. The mass vector $\overline{\mathbf{m}}_{(k)}$ in Eq. (54a) can be replaced by a mass stable vector $\mathbf{M}_{(k)}$, and the damping force is added into the PD equations of motion as [69]

$$
\mathbf{M}_{(k)}\left({ }^{t+\Delta t} \ddot{\mathbf{u}}_{(k)}\right)+\mathbf{C}_{(k)}\left({ }^{t} \dot{\mathbf{u}}_{(k)}\right)=\sum_{j=1}^{N} \mu_{(k)(j)}{ }_{0}^{t} \overline{\mathbf{f}}_{(k)(j)}{ }^{0} V_{(j)}+{ }^{t} \overline{\mathbf{b}}_{(k)}
$$

where $\mathbf{C}_{(k)}$ represents the damping matrix.

As proposed by Nguyen and Oterkus [27], the mass stable matrix, $\mathbf{M}_{(k)}$ for plates and shells can be defined as

$$
\mathbf{M}_{(k)}=\left[\begin{array}{ccccc}
M_{1} & 0 & 0 & 0 & 0 \\
0 & M_{1} & 0 & 0 & 0 \\
0 & 0 & M_{1} & 0 & 0 \\
0 & 0 & 0 & M_{2} & 0 \\
0 & 0 & 0 & 0 & M_{2}
\end{array}\right]
$$

with 


$$
\begin{aligned}
& M_{1}=\max \left(M_{u_{(k)}}, M_{v_{(k)}}, M_{w_{(k)}}\right) \\
& M_{2}=\max \left(M_{\theta_{x(k)}}, M_{\theta_{y(k)}}\right)
\end{aligned}
$$

where $M_{u_{(k)}}, M_{v_{(k)}}, M_{w_{(k)}}, M_{\theta_{x(k)}}, M_{\theta_{y(k)}}$ represent the components of the mass stable vector corresponding to translational and rotational degrees of freedom which can be estimated as [27, 75]

$$
\begin{aligned}
& M_{u_{(k)}}=M_{v_{(k)}} \geq \frac{1}{4} d t^{2} \frac{4 b_{i p} \pi h \delta^{2}}{\Delta x} \\
& M_{w_{(k)}} \geq \frac{1}{4} d t^{2} \frac{C_{s h} \pi h \delta^{2}}{\Delta x} \\
& M_{\theta_{x(k)}}=M_{\theta_{y(k)}} \geq \frac{1}{4} d t^{2} \frac{4 b_{b} \pi h \delta^{2}}{\Delta x}
\end{aligned}
$$

where $d t=1$ represents the time step for a quasi-static solution [68], $\Delta x$ represents mesh size. The PD constants, $b_{i p}, C_{s}, b_{b}$ are given in Eq. (44c), Eq. (46), Eq. (48c), respectively.

\section{Appendix E. Volume correction and surface correction}

In described in Section 6, the volume and surface correction factors are used in the calculation of PD force densities. Therefore, in this section, the calculations of volume and surface correction factors are presented.

\section{E.1. Volume correction factor}

As proposed by Silling [67] and later described by Madenci and Oterkus [8], the volume correction factor, $v_{(k)(j)}$, for the interaction between material points $k$ and $j$ can be calculated as

$$
v_{(k)(j)}=\left\{\begin{array}{lll}
1 & \text { if } & { }^{0} \xi_{(k)(j)} \leq(\delta-\Delta x / 2) \\
\left(\delta+\Delta x / 2-{ }^{0} \xi_{(k)(j)}\right) / \Delta x & \text { if } & (\delta-\Delta x / 2)<{ }^{0} \xi_{(k)(j)} \leq \delta \\
0 & \text { if } & { }^{0} \xi_{(k)(j)}>\delta
\end{array}\right.
$$

\section{E.2. Surface correction factors for in-plane deformations}

As proposed by Madenci and Oterkus [8], Madenci and Oterkus [16], the correction factors for dilatation terms given in Eq. (63a) can be calculated as

$$
D_{(k)(j)}^{(i p)}=\left(\left(\frac{\cos \varphi}{D_{x(k)(j)}^{(i p)}}\right)^{2}+\left(\frac{\sin \varphi}{D_{y(k)(j)}^{(i p)}}\right)^{2}\right)^{-1 / 2}
$$

with 


$$
\begin{aligned}
& D_{x(k)(j)}^{(i p)}=\frac{1}{2}\left(D_{x(k)}^{(i p)}+D_{x(j)}^{(i p)}\right) \\
& D_{y(k)(j)}^{(i p)}=\frac{1}{2}\left(D_{y(k)}^{(i p)}+D_{y(j)}^{(i p)}\right)
\end{aligned}
$$

where $D_{x(k)}^{(i p)}$ and $D_{x(j)}^{(i p)}$ represent the correction factors in $x$-direction for dilatations of material point $k$ and $j$, respectively. Meanwhile, $D_{y(k)}^{(i p)}$ and $D_{y(j)}^{(i p)}$ represent the correction factors in $y$ -direction for dilatations of material point $k$ and $j$, respectively. The terms $D_{x(k)(j)}^{(i p)}$ and $D_{y(k)(j)}^{(i p)}$ represent the average values of the correction factors in $x$-and $y$-direction for dilatations of the interaction between material points $k$ and $j$, respectively.

Similarly, the surface correction factor for in-plane deformations given in Eqs. (64a-c) and Eqs. $(65 \mathrm{a}-\mathrm{c})$ can be calculated as $[8,16]$

$$
\Omega_{(k)(j)}^{(i p)}=\left(\left(\frac{\cos \varphi}{\Omega_{x(k)(j)}^{(i p)}}\right)^{2}+\left(\frac{\sin \varphi}{\Omega_{y(k)(j)}^{(i p)}}\right)^{2}\right)^{-1 / 2}
$$

with

$$
\begin{aligned}
& \Omega_{x(k)(j)}^{(i p)}=\frac{1}{2}\left(\Omega_{x(k)}^{(i p)}+\Omega_{x(j)}^{(i p)}\right) \\
& \Omega_{y(k)(j)}^{(i p)}=\frac{1}{2}\left(\Omega_{y(k)}^{(i p)}+\Omega_{y(j)}^{(i p)}\right)
\end{aligned}
$$

where $\Omega_{x(k)}^{(i p)}$ and $\Omega_{x(j)}^{(i p)}$ represent the surface correction factors in $x$-direction for in-plane deformations of material point $k$ and $j$, respectively. Meanwhile, $\Omega_{y(k)}^{(i p)}$ and $\Omega_{y(j)}^{(i p)}$ represent the surface correction factors in $y$-direction for in-plane deformations of material point $k$ and $j$, respectively. The terms $\Omega_{x(k)(j)}^{(i p)}$ and $\Omega_{y(k)(j)}^{(i p)}$ represent the average values of the surface correction factors in $x$ - and $y$-direction for in-plane deformations of the interaction between material points $k$ and $j$, respectively.

To calculate the correction factors in $x$-direction, $D_{x(k)}^{(i p)}, D_{x(j)}^{(i p)}, \Omega_{x(k)}^{(i p)}$ and $\Omega_{x(j)}^{(i p)}$ given in Eq. (E2b) and Eq. (E3b), the following loading conditions are assumed [8]

$$
\begin{aligned}
& { }_{0}^{t} u_{, x}=\ell \ll 1, \quad{ }_{0} \underline{u}_{, x}=\Delta \ell \ll \ell \\
& { }_{0}^{t} u_{, y}={ }_{0}^{t} v_{, x}={ }_{0}^{t} v_{, y}={ }_{0}^{t} w_{, x}={ }_{0}^{t} w_{, y}=0 \\
& { }_{0} \underline{u}, y_{, y}={ }_{0} \underline{v}_{, x}={ }_{0} \underline{v}_{, y}={ }_{0} \underline{w}_{, x}={ }_{0} \underline{w}_{, y}=0 \\
& \underline{\theta}_{x}=\underline{\theta}_{y}={ }^{t} \theta_{x}={ }^{t} \theta_{y}=0
\end{aligned}
$$

where $\ell$ and $\Delta \ell$ are very small values such as $\ell=0.001$ and $\Delta \ell=10^{-5}$.

By using the loading conditions given in Eq. (E4), the dilatations in classical continuum mechanics given in Eqs. (34d-e) can be calculated as 
${ }_{0}^{t} \vartheta=\ell+\frac{1}{2} \ell^{2}$

$0 \underline{\vartheta}=\Delta \ell+\ell(\Delta \ell)$

On the other hand, the dilatation in peridynamics, ${ }_{0}^{t} \vartheta_{(k)}^{(P D)}$, can be calculated using Eq. (41d). Therefore, the correction factor in $x$-direction for dilatation, $D_{x(k)}^{(i p)}$, can be evaluated as [8]

$$
D_{x(k)}^{(i p)}=\frac{{ }_{0}^{t} \vartheta}{{ }_{0}^{t} \vartheta_{(k)}^{(P D)}}=\frac{\ell+\frac{1}{2} \ell^{2}}{d_{i p} \sum_{j=1}^{N}{ }_{0}^{t} s_{i p(k)(j)}{ }^{0} V_{(j)}}
$$

Similarly, by using the loading conditions given in Eq. (E4), the strain energy per unit area for in-plane deformations in classical continuum mechanics given in Eq. (35b) can be calculated as

$$
\bar{W}_{i p}^{N L}=\frac{E h}{1-v^{2}}\left(\ell+\frac{1}{2} \ell^{2}\right)(\Delta \ell+\ell(\Delta \ell))
$$

Meanwhile, the strain energy per unit area for in-plane deformations in peridynamics given in Eq. (41a) can be calculated as

$$
\bar{W}_{i p(k)}^{N L P D}=2 a_{i p}\left(\ell+\frac{1}{2} \ell^{2}\right)(\Delta \ell+\ell(\Delta \ell))+2 b_{i p} \sum_{j=1}^{N}\left({ }_{0}^{t} s_{i p(k)(j)}\right)\left({ }_{0} \underline{s}_{i p(k)(j)}\right){ }^{0} \xi_{(k)(j)}{ }^{0} V_{(j)}
$$

or

$$
\bar{W}_{i p(k)}^{N L P D}=2 a_{i p}\left(\ell+\frac{1}{2} \ell^{2}\right)(\Delta \ell+\ell(\Delta \ell))+\bar{W}_{i p(k)}^{N L P D(\mu)}
$$

with

$$
\bar{W}_{i p(k)}^{N L P D(\mu)}=2 b_{i p} \sum_{j=1}^{N}\left({ }_{0}^{t} s_{i p(k)(j)}\right)\left({ }_{0} \underline{s}_{i p(k)(j)}\right)^{0} \xi_{(k)(j)}{ }^{0} V_{(j)}
$$

Therefore, by comparing Eq. (E7) and Eq. (E8b), the surface correction factors in $x$-direction for in-plane deformations, $\Omega_{x(k)}^{(i p)}$, can be estimated as

$$
\Omega_{x(k)}^{(i p)}=\frac{\bar{W}_{i p}^{N L}-2 a_{i p}\left(\ell+\frac{1}{2} \ell^{2}\right)(\Delta \ell+\ell(\Delta \ell))}{\bar{W}_{i p(k)}^{N L P D(\mu)}}=\frac{\left(\frac{E h}{1-v^{2}}-2 a_{i p}\right)\left(\ell+\frac{1}{2} \ell^{2}\right)(\Delta \ell+\ell(\Delta \ell))}{2 b_{i p} \sum_{j=1}^{N}\left({ }_{0}^{t} s_{i p(k)(j)}\right)\left({ }_{0} \underline{s}_{i p(k)(j)}\right)^{0} \xi_{(k)(j)}{ }^{0} V_{(j)}}
$$

To calculate the correction factors in $y$-direction, $D_{y(k)}^{(i p)}, D_{y(j)}^{(i p)}, \Omega_{y(k)}^{(i p)}$ and $\Omega_{y(j)}^{(i p)}$ given in Eq. (E2c) and Eq. (E3c), the following loading conditions are assumed [8]

$$
\begin{aligned}
& { }_{0}^{t} v_{, y}=\ell \ll 1, \quad \underline{v}_{, y}=\Delta \ell \ll \ell \\
& { }_{0}^{t} u_{, x}={ }_{0}^{t} u_{, y}={ }_{0}^{t} v_{, x}={ }_{0}^{t} w_{, x}={ }_{0}^{t} w_{, y}=0
\end{aligned}
$$


${ }_{0} \underline{u}_{, x}={ }_{0} \underline{u}_{y}={ }_{0} \underline{v}, x_{, x}={ }_{0} \underline{w}_{, x}={ }_{0} \underline{w}_{, y}=0$

$\underline{\theta}_{x}=\underline{\theta}_{y}={ }^{t} \theta_{x}={ }^{t} \theta_{y}=0$

where $\ell$ and $\Delta \ell$ are very small values such as $\ell=0.001$ and $\Delta \ell=10^{-5}$.

The loading conditions given in Eq. (E10) yield the same values for dilatations and strain energy per unit area in classical continuum mechanics as given in Eq. (E5) and Eq. (E7), respectively. Therefore, the correction factors in $y$-direction can also be estimated as

$$
\begin{aligned}
& D_{y(k)}^{(i p)}=\frac{{ }_{0}^{t} \vartheta}{{ }_{0}^{t} \vartheta_{(k)}^{(P D)}}=\frac{\ell+\frac{1}{2} \ell^{2}}{d_{i p} \sum_{j=1}^{N}{ }_{0}^{t} s_{i p(k)(j)}{ }^{0} V_{(j)}} \\
& \Omega_{y(k)}^{(i p)}=\frac{\bar{W}_{i p}^{N L}-2 a_{i p}\left(\ell+\frac{1}{2} \ell^{2}\right)(\Delta \ell+\ell(\Delta \ell))}{\bar{W}_{i p(k)}^{N L P D(\mu)}}=\frac{\left(\frac{E h}{1-v^{2}}-2 a_{i p}\right)\left(\ell+\frac{1}{2} \ell^{2}\right)(\Delta \ell+\ell(\Delta \ell))}{2 b_{i p} \sum_{j=1}^{N}\left({ }_{0}^{t} s_{i p(k)(j)}\right)\left({ }_{0} \underline{s}_{i p(k)(j)}\right)^{0} \xi_{(k)(j)}{ }^{0} V_{(j)}}
\end{aligned}
$$

\section{E.2. Surface correction for shear deformations}

Similar to the surface correction forms provided in $[8,16]$, the surface correction factors for shear deformations, $\Omega_{(k)}^{(s h)}$ given in Eqs. (64c-e) and Eqs. (E65c-e) can be calculated as

$\Omega_{(k)(j)}^{(s h)}=\left(\left(\frac{\cos \varphi}{\Omega_{x(k)(j)}^{(s h)}}\right)^{2}+\left(\frac{\sin \varphi}{\Omega_{y(k)(j)}^{(s h)}}\right)^{2}\right)^{-1 / 2}$

with

$$
\begin{aligned}
& \Omega_{x(k)(j)}^{(s h)}=\frac{1}{2}\left(\Omega_{x(k)}^{(s h)}+\Omega_{x(j)}^{(s h)}\right) \\
& \Omega_{y(k)(j)}^{(s h)}=\frac{1}{2}\left(\Omega_{y(k)}^{(s h)}+\Omega_{y(j)}^{(s h)}\right)
\end{aligned}
$$

where $\Omega_{x(k)}^{(s h)}$ and $\Omega_{x(j)}^{(s h)}$ represent the surface correction factors in $x$-direction for shear deformations of material point $k$ and $j$, respectively. Meanwhile, $\Omega_{y(k)}^{(s h)}$ and $\Omega_{y(j)}^{(s h)}$ represent the surface correction factors in $y$-direction for shear deformations of material point $k$ and $j$ , respectively. The terms $\Omega_{x(k)(j)}^{(s h)}$ and $\Omega_{y(k)(j)}^{(s h)}$ represent the average values of the surface correction factors in $x$-and $y$-direction for shear deformations of the interaction between material points $k$ and $j$, respectively.

To calculate the surface correction factors in $x$-direction, $\Omega_{x(k)}^{(s h)}$ given in Eq. (E12b), the following loading conditions are assumed [8]

$$
{ }_{0}^{t} w_{, x}=\ell \ll 1, \quad \underline{w}_{, x}=\Delta \ell \ll \ell
$$


${ }_{0}^{t} u_{, x}={ }_{0}^{t} u_{, y}={ }_{0}^{t} v_{, x}={ }_{0}^{t} v_{, y}={ }_{0}^{t} w_{, y}=0$

${ }_{0} \underline{u}_{, x}={ }_{0} \underline{u}_{, y}={ }_{0} \underline{v}_{, x}={ }_{0} \underline{v}_{, y}={ }_{0} \underline{w}_{, y}=0$

$\underline{\theta}_{x}=\underline{\theta}_{y}={ }^{t} \theta_{x}={ }^{t} \theta_{y}=0$

where $\ell$ and $\Delta \ell$ are very small values such as $\ell=0.001$ and $\Delta \ell=10^{-5}$.

By using the loading conditions given in Eq. (E13), the strain energy per unit area for shear deformations in classical continuum mechanics given in Eq. (35c) can be calculated as

$\bar{W}_{s h}^{N L}=\frac{k_{s} E h}{2(1+v)} \ell(\Delta \ell)$

Meanwhile, the strain energy per unit area for shear deformations in peridynamics can be calculated using Eq. (45a). Therefore, the surface correction factor in $x$-direction for shear deformations of the interaction between material points $k$ and $j$ can be calculated as $[8,53]$

$S F_{x(k)}^{(s h)}=\frac{\left(\bar{W}_{s h}^{N L}\right)}{\left(\bar{W}_{s h(k)}^{N L P D}\right)}=\frac{\frac{k_{s} E h}{2(1+v)} \ell(\Delta \ell)}{\left(\bar{W}_{s h(k)}^{N L P D}\right)}$

To calculate the surface correction factors in $y$-direction, $S F_{y(k)}^{(s h)}$ given in Eq. (E12c), the following loading conditions are assumed [8]

${ }_{0}^{t} w_{, y}=\ell \ll 1, \quad{ }_{0} \underline{w}_{, y}=\Delta \ell \ll \ell$

${ }_{0}^{t} u_{, x}={ }_{0}^{t} u_{, y}={ }_{0}^{t} v_{, x}={ }_{0}^{t} v_{, y}={ }_{0}^{t} w_{, x}=0$

${ }_{0} \underline{u}_{, x}={ }_{0} \underline{u}_{, y}={ }_{0} \underline{v}_{, x}={ }_{0} \underline{v}_{, y}={ }_{0} \underline{w}_{, x}=0$

$\underline{\theta}_{x}=\underline{\theta}_{y}={ }^{t} \theta_{x}={ }^{t} \theta_{y}=0$

where $\ell$ and $\Delta \ell$ are very small values such as $\ell=0.001$ and $\Delta \ell=10^{-5}$.

The loading conditions given in Eq. (E16) yield the same value for strain energy per unit area in classical continuum mechanics as given in Eq. (E14). Therefore, the surface correction factor in $y$-direction can also be estimated as

$$
S F_{y(k)}^{(s h)}=\frac{\frac{k_{s} E h}{2(1+v)} \ell(\Delta \ell)}{\left(\bar{W}_{s h(k)}^{N L P D}\right)}
$$

where the strain energy per unit area for shear deformations in peridynamics, $\bar{W}_{s h(k)}^{N L P D}$, can be calculated using Eq. (45a). 


\section{E.3. Surface correction for bending deformations}

Similar to Eq. (E2a), the correction factor for dilatation terms of bending deformations of the interaction between material points $k$ and $j$ given in Eq. (E63b) can be calculated as

$$
D_{(k)(j)}^{(b)}=\left(\left(\frac{\cos \varphi}{D_{x(k)(j)}^{(b)}}\right)^{2}+\left(\frac{\sin \varphi}{D_{y(k)(j)}^{(b)}}\right)^{2}\right)^{-1 / 2}
$$

with

$$
\begin{aligned}
& D_{x(k)(j)}^{(b)}=\frac{1}{2}\left(D_{x(k)}^{(b)}+D_{x(j)}^{(b)}\right) \\
& D_{y(k)(j)}^{(b)}=\frac{1}{2}\left(D_{y(k)}^{(b)}+D_{y(j)}^{(b)}\right)
\end{aligned}
$$

where $D_{x(k)}^{(b)}$ and $D_{x(j)}^{(b)}$ represent the correction factors in $x$-direction for dilatation terms for bending deformations of material point $k$ and $j$, respectively. Meanwhile, $D_{y(k)}^{(b)}$ and $D_{y(j)}^{(b)}$ represent the correction factors in $y$-direction for dilatation terms for bending deformations of material point $k$ and $j$, respectively. The terms $D_{x(k)(j)}^{(b)}$ and $D_{y(k)(j)}^{(b)}$ represent the average values of the correction factors in $x$ - and $y$-direction for dilatation terms for bending deformations of the interaction between material points $k$ and $j$, respectively.

Similar to Eq. (E12a), the surface correction factor for bending deformations of the interaction between material points $k$ and $j$ given in Eqs. (64d-e) and Eqs. (65d-e) can be calculated as

$\Omega_{(k)(j)}^{(b)}=\left(\left(\frac{\cos \varphi}{\Omega_{x(k)(j)}^{(b)}}\right)^{2}+\left(\frac{\sin \varphi}{\Omega_{y(k)(j)}^{(b)}}\right)^{2}\right)^{-1 / 2}$

with

$$
\begin{aligned}
& \Omega_{x(k)(j)}^{(b)}=\frac{1}{2}\left(\Omega_{x(k)}^{(b)}+\Omega_{x(j)}^{(b)}\right) \\
& \Omega_{y(k)(j)}^{(b)}=\frac{1}{2}\left(\Omega_{y(k)}^{(b)}+\Omega_{y(j)}^{(b)}\right)
\end{aligned}
$$

where $\Omega_{x(k)}^{(b)}$ and $\Omega_{x(j)}^{(b)}$ represent the surface correction factors in $x$-direction for bending deformations of material point $k$ and $j$, respectively. Meanwhile, $\Omega_{y(k)}^{(b)}$ and $\Omega_{y(j)}^{(b)}$ represent the surface correction factors in $y$-direction for bending deformations of material point $k$ and $j$, respectively. The terms $\Omega_{x(k)(j)}^{(b)}$ and $\Omega_{y(k)(j)}^{(b)}$ represent the average values of the surface correction factors in $x$-and $y$-direction for bending deformations of the interaction between material points $k$ and $j$, respectively.

To calculate the correction factors in $x$-direction, $D_{x(k)}^{(b)}, D_{x(j)}^{(b)}, \Omega_{x(k)}^{(b)}$ and $\Omega_{x(j)}^{(b)}$ given in Eq. (E18b) and Eq. (E19b), the following loading conditions are assumed [8] 
${ }_{0}^{t} \theta_{y, x}=\ell \ll 1,{ }_{0} \theta_{y, x}=\Delta \ell \ll \ell$

${ }_{0}^{t} u_{, x}={ }_{0}^{t} u_{, y}={ }_{0}^{t} v_{, x}={ }_{0}^{t} v_{, y}={ }_{0}^{t} w_{, x}={ }_{0}^{t} w_{, y}=0$

${ }_{0} \underline{u}, x_{,}={ }_{0} \underline{u}_{, y}={ }_{0} \underline{v}_{, x}={ }_{0} \underline{\underline{v}}_{, y}={ }_{0} \underline{w}_{, x}={ }_{0} \underline{w}_{, y}=0$

${ }_{0}^{t} \theta_{x, x}={ }_{0}^{t} \theta_{x, y}={ }_{0}^{t} \theta_{y, y}={ }_{0} \underline{\theta}_{x, y}={ }_{0} \underline{\theta}_{x, x}={ }_{0} \underline{\theta}_{y, y}=0$

where $\ell$ and $\Delta \ell$ are very small values such as $\ell=0.001$ and $\Delta \ell=10^{-5}$.

By using the loading conditions given in Eq. (E20), the dilatation term for bending deformations in classical continuum mechanics, given in Eq. (36c-d), can be calculated as

${ }_{0}^{t} \vartheta_{b}=-\ell$

${ }_{0} \underline{\vartheta}_{b}=-\Delta \ell$

Meanwhile, the dilatation for bending deformations in peridynamics, ${ }_{0}^{t} \vartheta_{b(k)}^{(P D)}$, can be calculated by using Eq. (47b). Therefore, the correction factor in $x$-direction for dilatation for bending deformations, $D_{x(k)}^{(b)}$, can be evaluated as [8]

$D_{x(k)}^{(b)}=\frac{{ }_{0}^{t} \vartheta_{b}}{{ }_{0} \vartheta_{b(k)}^{(P D)}}=\frac{-\ell}{d_{b} \sum_{j=1}^{N}{ }_{0}^{t} s_{b(k)(j)}{ }^{0} V_{(j)}}$

By using the loading conditions given in Eq. (E20), the strain energy per unit area in classical continuum mechanics and peridynamics can be calculated using Eq. (36b) and Eq. (47a) as

$$
\begin{aligned}
& \bar{W}_{b}^{N L}=\frac{E h^{3}}{12\left(1-v^{2}\right)} \ell(\Delta \ell) \\
& \bar{W}_{b(k)}^{N L P D}=2 a_{b} \ell(\Delta \ell)+2 b_{b} \sum_{j=1}^{N}\left({ }_{0}^{t} s_{b(k)(j)}\right)\left({ }_{0} \underline{s}_{b(k)(j)}\right)^{0} \xi_{(k)(j)}{ }^{0} V_{(j)}
\end{aligned}
$$

Therefore, by comparing Eq. (E23a) and Eq. (E23b), the surface correction factors in $x$ direction for bending deformations, $\Omega_{x(k)}^{(b)}$, can be estimated as

$$
\Omega_{x(k)}^{(b)}=\frac{\bar{W}_{b}^{N L}-2 a_{b} \ell(\Delta \ell)}{2 b_{b} \sum_{j=1}^{N}\left({ }_{0}^{t} s_{b(k)(j)}\right)\left({ }_{0} \underline{s}_{b(k)(j)}\right){ }^{0} \xi_{(k)(j)}{ }^{0} V_{(j)}}=\frac{\left(\frac{E h^{3}}{12\left(1-v^{2}\right)}-2 a_{b}\right) \ell(\Delta \ell)}{2 b_{b} \sum_{j=1}^{N}\left({ }_{0}^{t} s_{b(k)(j)}\right)\left({ }_{0} \underline{s}_{b(k)(j)}\right){ }^{0} \xi_{(k)(j)}{ }^{0} V_{(j)}}
$$

To calculate the correction factors in $y$-direction, $D_{y(k)}^{(b)}, D_{y(j)}^{(b)}, \Omega_{y(k)}^{(b)}$ and $\Omega_{y(j)}^{(b)}$ given in Eq. (E18c) and Eq. (E19c), the following loading conditions are assumed [8]

$$
\begin{aligned}
& { }_{0}^{t} \theta_{x, y}=\ell \ll 1, \quad{ }_{0} \theta_{x, y}=\Delta \ell \ll \ell \\
& { }_{0}^{t} u_{, x}={ }_{0}^{t} u_{, y}={ }_{0}^{t} v_{, x}={ }_{0}^{t} v_{, y}={ }_{0}^{t} w_{, x}={ }_{0}^{t} w_{, y}=0
\end{aligned}
$$


${ }_{0} \underline{u}, x={ }_{0} \underline{u}_{, y}={ }_{0} \underline{v}_{, x}={ }_{0} \underline{\underline{v}}_{, y}={ }_{0} \underline{w}_{, x}={ }_{0} \underline{w}_{, y}=0$

${ }_{0}^{t} \theta_{x, x}={ }_{0}^{t} \theta_{y, x}={ }_{0}^{t} \theta_{y, y}={ }_{0} \underline{\theta}_{x, x}={ }_{0} \underline{\theta}_{y, x}={ }_{0} \underline{\theta}_{y, y}=0$

where $\ell$ and $\Delta \ell$ are very small values such as $\ell=0.001$ and $\Delta \ell=10^{-5}$.

The loading conditions given in Eq. (E25) yield the same value of strain energy per unit area for bending deformations in classical continuum mechanics as given Eq. (E23). Therefore, the surface correction factors in $y$-direction for bending deformations can also be estimated as

$\Omega_{y(k)}^{(b)}=\frac{\bar{W}_{b}^{N L}-2 a_{b} \ell(\Delta \ell)}{2 b_{b} \sum_{j=1}^{N}\left({ }_{0}^{t} s_{b(k)(j)}\right)\left({ }_{0} \underline{s}_{b(k)(j)}\right){ }^{0} \xi_{(k)(j)}{ }^{0} V_{(j)}}=\frac{\left(\frac{E h^{3}}{12\left(1-v^{2}\right)}-2 a_{b}\right) \ell(\Delta \ell)}{2 b_{b} \sum_{j=1}^{N}\left({ }_{0}^{t} s_{b(k)(j)}\right)\left({ }_{0} \underline{s}_{b(k)(j)}\right){ }^{0} \xi_{(k)(j)}{ }^{0} V_{(j)}}$

Meanwhile, by using the loading conditions given in Eq. (E25), the dilatation term for bending deformations in classical continuum mechanics, given in Eq. (36c), can be calculated as

${ }_{0}^{t} \vartheta_{b}=\ell$

Therefore, the correction factor in $y$-direction for dilatation for bending deformations, $D_{y(k)}^{(b)}$, can be evaluated as [8]

$$
D_{y(k)}^{(b)}=\frac{\ell}{d_{b} \sum_{j=1}^{N}{ }_{0}^{t} s_{b(k)(j)}{ }^{0} V_{(j)}}
$$




\section{References}

[1] K.-J. Bathe, Finite element procedures, Klaus-Jurgen Bathe, 2006.

[2] T. Sussman, K.-J. Bathe, 3D-shell elements for structures in large strains, Computers \& structures, 122 (2013) 2-12.

[3] K.-j. Bathe, S. Bolourchi, A geometric and material nonlinear plate and shell element, Computers \& Structures, 11 (1980) 23-48.

[4] S.A. Silling, Reformulation of elasticity theory for discontinuities and long-range forces, Journal of the Mechanics and Physics of Solids, 48 (2000) 175-209.

[5] S.A. Silling, M. Epton, O. Weckner, J. Xu, E. Askari, Peridynamic states and constitutive modeling, Journal of Elasticity, 88 (2007) 151-184.

[6] S.A. Silling, R. Lehoucq, Peridynamic theory of solid mechanics, in: Advances in applied mechanics, Elsevier, 2010, pp. 73-168.

[7] S.A. Silling, E. Askari, A meshfree method based on the peridynamic model of solid mechanics, Computers \& structures, 83 (2005) 1526-1535.

[8] E. Madenci, E. Oterkus, Peridynamic Theory and Its Applications, Springer, 2014.

[9] Y.D. Ha, F. Bobaru, Studies of dynamic crack propagation and crack branching with peridynamics, International Journal of Fracture, 162 (2010) 229-244.

[10] F. Bobaru, W. Hu, The meaning, selection, and use of the peridynamic horizon and its relation to crack branching in brittle materials, International journal of fracture, 176 (2012) 215-222.

[11] A. Agwai, I. Guven, E. Madenci, Predicting crack propagation with peridynamics: a comparative study, International journal of fracture, 171 (2011) 65.

[12] B. Kilic, E. Madenci, Prediction of crack paths in a quenched glass plate by using peridynamic theory, International journal of fracture, 156 (2009) 165-177.

[13] Y.D. Ha, F. Bobaru, Characteristics of dynamic brittle fracture captured with peridynamics, Engineering Fracture Mechanics, 78 (2011) 1156-1168.

[14] C.T. Nguyen, S. Oterkus, Ordinary state-based peridynamic model for geometrically nonlinear analysis, Engineering Fracture Mechanics, (2019).

[15] J.T. Foster, S.A. Silling, W.W. Chen, Viscoplasticity using peridynamics, International journal for numerical methods in engineering, 81 (2010) 1242-1258.

[16] E. Madenci, S. Oterkus, Ordinary state-based peridynamics for plastic deformation according to von Mises yield criteria with isotropic hardening, Journal of the Mechanics Physics of Solids, 86 (2016) 192-219.

[17] Y. Huang, S. Oterkus, H. Hou, E. Oterkus, Z. Wei, S. Zhang, Peridynamic model for visco-hyperelastic material deformation in different strain rates, Continuum Mechanics Thermodynamics, (2019) 1-35.

[18] E. Madenci, S. Oterkus, Ordinary state-based peridynamics for thermoviscoelastic deformation, Engineering Fracture Mechanics, 175 (2017) 31-45.

[19] S. Oterkus, E. Madenci, A. Agwai, Fully coupled peridynamic thermomechanics, Journal of the Mechanics Physics of Solids, 64 (2014) 1-23.

[20] S. Oterkus, E. Madenci, E. Oterkus, Fully coupled poroelastic peridynamic formulation for fluid-filled fractures, Engineering geology, 225 (2017) 19-28.

[21] Y. Gao, S. Oterkus, Fully coupled thermomechanical analysis of laminated composites by using ordinary state based peridynamic theory, Composite Structures, 207 (2019) 397-424.

[22] Y. Gao, S. Oterkus, Non-local modeling for fluid flow coupled with heat transfer by using peridynamic differential operator, Engineering Analysis with Boundary Elements, 105 (2019) 104-121.

[23] C. Diyaroglu, S. Oterkus, E. Oterkus, E. Madenci, Peridynamic modeling of diffusion by using finite-element analysis, IEEE Transactions on Components, Packaging Manufacturing Technology, 7 (2017) 1823-1831. 
[24] H. Wang, E. Oterkus, S. Oterkus, Peridynamic modelling of fracture in marine lithiumion batteries, Ocean Engineering, 151 (2018) 257-267.

[25] E. Madenci, S. Oterkus, Peridynamics for coupled field equations, Handbook of Peridynamic Modeling, (2016) 489-531.

[26] S. Oterkus, Peridynamics for the solution of multiphysics problems, in: Aerospace and Mechanical Engineering Faculty, The University of Arizona, 2015.

[27] C.T. Nguyen, S. Oterkus, Peridynamics for the thermomechanical behavior of shell structures, Engineering Fracture Mechanics, (2019) 106623.

[28] H. Wang, E. Oterkus, S. Oterkus, Predicting fracture evolution during lithiation process using peridynamics, Engineering Fracture Mechanics, 192 (2018) 176-191.

[29] F. Bobaru, Y.D. Ha, Adaptive refinement and multiscale modeling in 2D peridynamics, Journal for Multiscale Computational Engineering, (2011) 635-659.

[30] E. Askari, F. Bobaru, R. Lehoucq, M. Parks, S. Silling, O. Weckner, Peridynamics for multiscale materials modeling, in: Journal of Physics: Conference Series, IOP Publishing, 2008, pp. 012078.

[31] S. Jafarzadeh, Z. Chen, S. Li, F. Bobaru, A peridynamic mechano-chemical damage model for stress-assisted corrosion, Electrochimica Acta, 323 (2019) 134795.

[32] E. Oterkus, E. Madenci, O. Weckner, S. Silling, P. Bogert, A. Tessler, Combined finite element and peridynamic analyses for predicting failure in a stiffened composite curved panel with a central slot, Composite Structures, 94 (2012) 839-850.

[33] W. Hu, Y.D. Ha, F. Bobaru, Peridynamic model for dynamic fracture in unidirectional fiber-reinforced composites, Computer Methods in Applied Mechanics Engineering, 217 (2012) 247-261.

[34] E. Oterkus, E. Madenci, Peridynamic theory for damage initiation and growth in composite laminate, in: Key Engineering Materials, Trans Tech Publ, 2012, pp. 355-358.

[35] E. Oterkus, Peridynamic theory for modeling three-dimensional damage growth in metallic and composite structures, in, The University of Arizona, The University of Arizona, 2010.

[36] S.A. Silling, A. Askari, Peridynamic model for fatigue cracking, SAND-18590. Albuquerque: Sandia National Laboratories, (2014).

[37] G. Zhang, Q. Le, A. Loghin, A. Subramaniyan, F. Bobaru, Validation of a peridynamic model for fatigue cracking, Engineering Fracture Mechanics, 162 (2016) 76-94.

[38] J. Jung, J. Seok, Mixed-mode fatigue crack growth analysis using peridynamic approach, International Journal of Fatigue, 103 (2017) 591-603.

[39] W. Liu, J.-W. Hong, A coupling approach of discretized peridynamics with finite element method, Computer methods in applied mechanics engineering, 245 (2012) 163-175.

[40] Y. Bie, X. Cui, Z. Li, A coupling approach of state-based peridynamics with node-based smoothed finite element method, Computer Methods in Applied Mechanics Engineering, 331 (2018) 675-700.

[41] B. Kilic, E. Madenci, Coupling of peridynamic theory and the finite element method, Journal of mechanics of materials structures, 5 (2010) 707-733.

[42] Z. Yang, E. Oterkus, C.T. Nguyen, S. Oterkus, Implementation of peridynamic beam and plate formulations in finite element framework, Continuum Mechanics Thermodynamics, 31 (2019) 301-315.

[43] R.W. Macek, S.A. Silling, Peridynamics via finite element analysis, Finite Elements in Analysis Design, 43 (2007) 1169-1178.

[44] E. Madenci, M. Dorduncu, A. Barut, N. Phan, A state-based peridynamic analysis in a finite element framework, Engineering Fracture Mechanics, 195 (2018) 104-128. 
[45] T. Ni, M. Zaccariotto, Q.-Z. Zhu, U. Galvanetto, Coupling of FEM and ordinary statebased peridynamics for brittle failure analysis in 3D, Mechanics of Advanced Materials and Structures, (2019) 1-16.

[46] T. Ni, F. Pesavento, M. Zaccariotto, U. Galvanetto, Q.-Z. Zhu, B.A. Schrefler, Hybrid FEM and peridynamic simulation of hydraulic fracture propagation in saturated porous media, Computer Methods in Applied Mechanics and Engineering, 366 (2020) 113101.

[47] M. Breitenfeld, P.H. Geubelle, O. Weckner, S. Silling, Non-ordinary state-based peridynamic analysis of stationary crack problems, Computer Methods in Applied Mechanics Engineering, 272 (2014) 233-250.

[48] E. Madenci, Peridynamic integrals for strain invariants of homogeneous deformation, ZAMM-Journal of Applied Mathematics and Mechanics/Zeitschrift für Angewandte Mathematik und Mechanik, 97 (2017) 1236-1251.

[49] G. Sarego, Q.V. Le, F. Bobaru, M. Zaccariotto, U. Galvanetto, Linearized state-based peridynamics for 2-D problems, International Journal for Numerical Methods in Engineering, 108 (2016) 1174-1197.

[50] S.A. Silling, M. Zimmermann, R. Abeyaratne, Deformation of a peridynamic bar, Journal of Elasticity, 73 (2003) 173-190.

[51] J. O'Grady, J. Foster, Peridynamic beams: a non-ordinary, state-based model, International Journal of Solids Structures, 51 (2014) 3177-3183.

[52] C. Diyaroglu, E. Oterkus, S. Oterkus, An Euler-Bernoulli beam formulation in an ordinary state-based peridynamic framework, Mathematics Mechanics of Solids, 24 (2019) 361-376.

[53] C. Diyaroglu, E. Oterkus, S. Oterkus, E. Madenci, Peridynamics for bending of beams and plates with transverse shear deformation, International Journal of Solids Structures, 69 (2015) 152-168.

[54] C.T. Nguyen, S. Oterkus, Peridynamics formulation for beam structures to predict damage in offshore structures, Ocean Engineering, 173 (2019) 244-267.

[55] S.A. Silling, F. Bobaru, Peridynamic modeling of membranes and fibers, International Journal of Non-Linear Mechanics, 40 (2005) 395-409.

[56] J. O'Grady, J. Foster, Peridynamic plates and flat shells: A non-ordinary, state-based model, International Journal of Solids Structures, 51 (2014) 4572-4579.

[57] C.T. Nguyen, S. Oterkus, Investigating the effect of crack propagation on strength of ship structures by using peridynamics, Manuscript submitted for publication.

[58] M. Dorduncu, Stress analysis of sandwich plates with functionally graded cores using peridynamic differential operator and refined zigzag theory, Thin-Walled Structures, 146 (2020) 106468.

[59] M. Dorduncu, K. Kaya, O.F. Ergin, Peridynamic Analysis of Laminated Composite Plates Based on First-Order Shear Deformation Theory, International Journal of Applied Mechanics, (2020) 2050031.

[60] E. Madenci, A. Barut, M. Dorduncu, Peridynamic differential operator for numerical analysis, Springer, 2019.

[61] E. Madenci, A. Barut, M. Futch, Peridynamic differential operator and its applications, Computer Methods in Applied Mechanics and Engineering, 304 (2016) 408-451.

[62] R.D. Mindlin, Influence of rotatory inertia and shear on flexural motions of isotropic, elastic plates, J. appl. Mech., 18 (1951) 31-38.

[63] E. Reissner, The effect of transverse shear deformation on the bending of elastic plates, J. appl. Mech., (1945) A69-A77.

[64] A. Barut, Nonlinear thermo-mechanical analysis of stiffened composite laminates by a new finite element, in: Aerospace and Mechanical Engineering Faculty, The University of Arizona, 1998. 
[65] J.T. Foster, S.A. Silling, W. Chen, An energy based failure criterion for use with peridynamic states, International Journal for Multiscale Computational Engineering, 9 (2011). [66] C.T. Nguyen, S. Oterkus, Investigating the effect of brittle crack propagation on the strength of ship structures by using peridynamics, Ocean Engineering, 209 (2020) 107472.

[67] S. Silling, EMU user's manual, Code Ver. 2.6 d, Sandia National Laboratories, Albuquerque, (2004).

[68] P. Underwood, Dynamic relaxation, Computational method for transient analysis, 1 (1986) 245-263.

[69] B. Kilic, E. Madenci, An adaptive dynamic relaxation method for quasi-static simulations using the peridynamic theory, Theoretical Applied Fracture Mechanics, 53 (2010) 194-204.

[70] C. Muscat-Fenech, A. Atkins, Out-of-plane stretching and tearing fracture in ductile sheet materials, International Journal of Fracture, 84 (1997) 297-306.

[71] P. Areias, T. Rabczuk, M. Msekh, Phase-field analysis of finite-strain plates and shells including element subdivision, Computer Methods in Applied Mechanics and Engineering, 312 (2016) 322-350.

[72] P. Areias, T. Rabczuk, Finite strain fracture of plates and shells with configurational forces and edge rotations, International Journal for Numerical Methods in Engineering, 94 (2013) 1099-1122.

[73] Y.-W. Mai, H. He, R. Leung, R.S. Seth, In-plane fracture toughness measurement of paper, in: Fracture Mechanics: 26th Volume, ASTM International, 1995.

[74] P.D. Zavattieri, Modeling of crack propagation in thin-walled structures using a cohesive model for shell elements, Journal of Applied Mechanics, (2006).

[75] C. Diyaroglu, Peridynamics and its applications in marine structures, in, University of Strathclyde, 2016. 